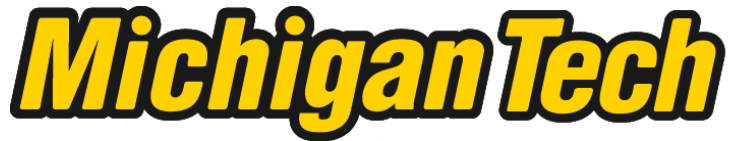 \\ Michigan Technological University Create the Future Digital Commons @ Michigan Tech
}

2013

\section{Influence of Mechanical and Thermal Boundary Conditions on Stabilizing/Destabilizing Mechanisms in Evaporating Liquid Films}

Aneet Dharmavaram Narendranath

Michigan Technological University

Follow this and additional works at: https://digitalcommons.mtu.edu/etds

Part of the Applied Mathematics Commons, and the Mechanical Engineering Commons Copyright 2013 Aneet Dharmavaram Narendranath

Recommended Citation

Dharmavaram Narendranath, Aneet, "Influence of Mechanical and Thermal Boundary Conditions on Stabilizing/Destabilizing Mechanisms in Evaporating Liquid Films", Dissertation, Michigan Technological University, 2013.

https://doi.org/10.37099/mtu.dc.etds/640

Follow this and additional works at: https://digitalcommons.mtu.edu/etds

Part of the Applied Mathematics Commons, and the Mechanical Engineering Commons 
INFLUENCE OF MECHANICAL AND THERMAL BOUNDARY CONDITIONS ON STABILIZING/DESTABILIZING MECHANISMS IN EVAPORATING LIQUID FILMS

By

Aneet Dharmavaram Narendranath

\begin{abstract}
A DISSERTATION
Submitted in partial fulfillment of the requirements for the degree of DOCTOR OF PHILOSOPHY

Mechanical Engineering - Engineering Mechanics

MICHIGAN TECHNOLOGICAL UNIVERSITY

2013
\end{abstract}

Copyright (c) 2013 Aneet Dharmavaram Narendranath 
This dissertation has been approved in partial fulfillment of the requirements for the Degree of DOCTOR OF PHILOSOPHY in Mechanical Engineering - Engineering Mechanics.

Department of Mechanical Engineering - Engineering Mechanics

Dissertation Advisor:

Dr. Jeffrey S. Allen

Committee Member:

Dr. Amitabh Narain

Committee Member:

Dr. Fernando L. Ponta

Committee Member:

Dr. Robert W. Kolkka

Department Chair:

Professor William W. Predebon 


\section{Contents}

List of Figures . . . . . . . . . . . . . . . . . . . . . . . . . xiii

List of Tables . . . . . . . . . . . . . . . . . . . . . . . xiv

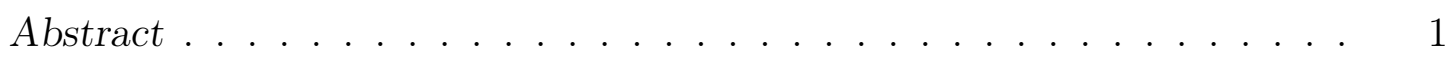

1. Stability of Liquid Films . . . . . . . . . . . . . . . . . . . . . . . . . . . . . . . . . . . . . . . .

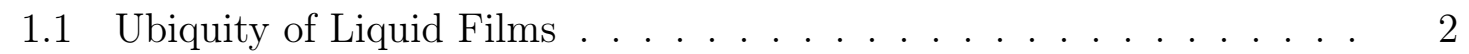

1.2 Destabilizing and Stabilizing Mechanisms . . . . . . . . . . . . . . . . 2

2. Inclusion Boussinesq Approximation . . . . . . . . . . . . . . . . . . . . . 8

2.1 Boussinesq Approximation and Scaling . . . . . . . . . . . . . . . . . 8

2.2 Interfacial Jump Conditions and Scaling . . . . . . . . . . . . . . . . 9

2.2.1 Jump Mass Balance. . . . . . . . . . . . . . . . . . . . . . . . 9

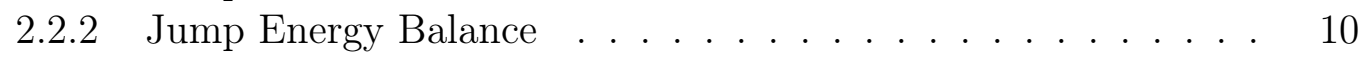

2.2 .3 Normal Stress Jump Condition . . . . . . . . . . . . . . . . . 10

2.2 .4 Shear Stress Jump Condition . . . . . . . . . . . . . . . . . . 10

2.2 .5 Reducing the Jump Conditions to One-Sided Model . . . . . . 11

$2.2 .6 \quad$ Scaling Interfacial Jump Conditions . . . . . . . . . . . . . . . 11

2.3 Rescaling . . . . . . . . . . . . . . . . . . . . . . . . 12

2.4 Alternative Formulations for Non-Evaporating Films . . . . . . . . . 15

3. Maximizing Wavelength . . . . . . . . . . . . . . . . . . . . . . . 17

3.1 Validity of Maximizing Wavenumber . . . . . . . . . . . . . . . . . . 19

3.2 Rescaling to Introduce Bond Number . . . . . . . . . . . . . . . . . . 19

4. Validation and Preliminary Results . . . . . . . . . . . . . . . . . . . . . . 21

4.1 Secondary Structures . . . . . . . . . . . . . . . . . . . . . . . . . . . . . . . . . . . . . . .

4.2 Definition of Rupture . . . . . . . . . . . . . . . . . . . . . . . 22 
5. Findings $\ldots \ldots \ldots \ldots \ldots \ldots$

$5.1 \quad$ Mathematical Fluid in Terrestrial \& Zero Gravity . . . . . . . . . 26

5.1 .1 Maximizing Wavelength $\ldots \ldots \ldots \ldots$. . . . . . . . . 27

5.1.2 Non-Evaporating Mathematical Fluid - Terrestrial Gravity . . 30

5.1.3 Slowly Evaporating Mathematical Fluid - Terrestrial Gravity. 31

$5.1 .4 \quad$ Slowly Evaporating Mathematical Fluid - Zero Gravity . . . . 32

5.1 .5 Zero Gravity Summary . . . . . . . . . . . . . . . . . . 33

5.2 Effects of Initial Conditions - Mathematical Fluid . . . . . . . . . . 34

5.2 .1 Perturbation Functions . . . . . . . . . . . . . . . . . . 34

5.2 .2 Changing Amplitude with a Fixed Domain Size . . . . . . . 34

5.2 .3 Changing Domain Size with a Fixed Amplitude . . . . . . . . 36

$5.2 .4 \quad$ Initial Condition Summary . . . . . . . . . . . . . . . . . 37

5.3 Dichloromethane (DCM) in Terrestrial \& Zero Gravity . . . . . . . . 39

5.3 .1 DCM Summary . . . . . . . . . . . . . . . . . . . . . . . . . 39

5.4 Noisy Uniform Initial Conditions - DCM in Zero Gravity . . . . . . . 41

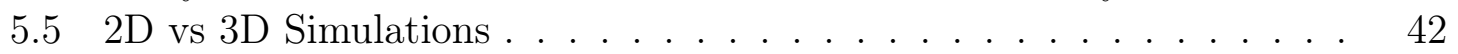

$5.5 .1 \quad$ 2D vs 3D Simulations Summary . . . . . . . . . . . . . 44

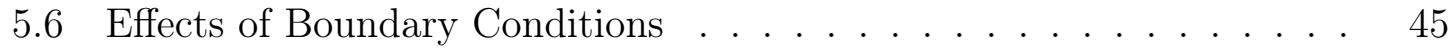

5.6 .1 Periodic vs Free and Pinned Boundary Conditions . . . . . . . 45

$5.6 .2 \quad$ Free Boundary Condition Summary . . . . . . . . . . . . 45

5.7 Comparison of Evolution Equations . . . . . . . . . . . . . . 47

5.7 .1 Evolution Equation Summary $\ldots \ldots \ldots \ldots$

6. Contributions to the field . . . . . . . . . . . . . . . . 48

\begin{tabular}{ll}
\hline Appendices & 50
\end{tabular}

A. Modified Constitutive Relationship . . . . . . . . . . . . . . . . . 51

B. Compendium of Results . . . . . . . . . . . . . . . . . . . 52

References . . . . . . . . . . . . . . . . . . . . 121 


\section{List OF FIGURES}

1.1 Various stabilizing and destabilizing forces that affect an evaporating liquid film. . . . . . . . . . . . . . . 3

4.1 When $\lambda=\lambda_{\max }$ and $\mathrm{M}=35.1, \mathrm{Pr}=7.20, \mathrm{~S}=100, \mathrm{G}=1 / 3$, rupture takes place at $\mathrm{t}=1280.0$ (within 1\% of CFD results [1]). $\ldots \ldots \ldots$. . . . . . 23

4.2 When $\lambda=2 \lambda_{\max }$ and $\mathrm{M}=35.1, \mathrm{Pr}=7.20, \mathrm{~S}=100, \mathrm{G}=1 / 3$, rupture takes place at $\mathrm{t}=2475.0$ (within $1 \%$ of CFD results [1]). . . . . . . . . . . 23

4.3 When $\lambda=4 \lambda_{\max }$ and $\mathrm{M}=35.1, \mathrm{Pr}=7.20, \mathrm{~S}=100, \mathrm{G}=1 / 3$, rupture takes place at $\mathrm{t}=4613.0$ (within $1 \%$ error of CFD results [1]).

4.4 Non-evaporating liquid film under the effect of surface tension $(S=$ $100)$, gravity, $(G=1 / 3)$ and thermocapillarity $(M=35.1)$ as in Oron [2]. With $\lambda=2 \lambda_{\max }$, the rupture time is revealed to be 2023.0. This is within $5 \%$ of that published by Oron et al. The contour plot is qualitatively the same as that of Oron [2] and is depicted here merely for a full comparison with Oron's results. . . . . . . . . . . . . . 25

4.5 With $\lambda=4 \lambda_{\max }$, the rupture time is revealed to be 3765.0. This is again within $5 \%$ of that published by Oron [2]. The contour plot is qualitatively the same and is depicted here merely for a full comparison with Oron's results. . . . . . . . . . . . . . . . . . . . . 25

5.1 Example of how a DFT plot represents wavelength dynamics . . . . . 26

5.2 DFT plot on the left corresponds to a non-evaporating liquid film in terrestrial gravity. Roughly $40 \%$ of the energy is situated in the fastest growing wavelength. In zero gravity (DFT plot on right), more energy (about 50\%) is stored in the fastest growing wavelength due to the absence of gravity stabilization. The zero gravity case also shows a cascade of energies. . . . . . . . . . . . . . . . 27

5.3 Non-dimensional maximizing wavelength plotted as a function of non dimensional film thickness, $h$, for an evaporating liquid film for "slow evaporation", $E=0.0001$. Dashed line is zero gravity, $G=0$ and solid line is when $G=1 / 3 . \ldots \ldots \ldots$. . . . . . . . . . . . 28 
5.4 Ratio of non-dimensional maximizing wavelengths $\lambda_{\max } / \lambda_{\max , \text { zero g }}$ plotted as a function of non dimensional film thickness, $h$, for an evaporating liquid film for "slow evaporation", $E=0.0001$. . . . . . . . . 29

5.5 Non-dimensional maximizing wavelength plotted as a function of non dimensional film thickness, $h$, for an evaporating liquid film for "fast evaporation", $E=0.1$. Dashed line is zero gravity, $G=0$ and solid line is when $G=1 / 3$. . . . . . . . . . . . . . . . . . . . . . . 29

5.6 Ratio of non-dimensional maximizing wavelengths $\lambda_{\max } / \lambda_{\max , \text { zero g }}$ plotted as a function of non dimensional film thickness, $h$, for an evaporating liquid film for "fast evaporation", $E=0.1$. . . . . . . . . . . . 29

5.7 Left is Oron's case [2]; right is the same case with $G=0.0$. Rupture times are 2032.0 and 1700.8 , respectively. . . . . . . . . . . . . 30

5.8 Left is Oron's case [2]; right is the same case with $G=0.0$. Rupture times are 3765.0 and 3435.8, respectively. . . . . . . . . . . . . . . 31

5.9 As the domain size is increased from $L=\lambda_{\max }$ (left) to $L=2 \lambda_{\max }$

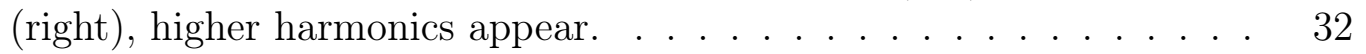

5.10 The effect of vapor recoil. Figure on left is the mathematical fluid evaporating in non-zero gravity $(\mathrm{G}=0.333)$. Figure on the right is the mathematical fluid evaporating in zero gravity $(\mathrm{G}=0.0)$. Zero gravity evaporation allows for thermocapillary structures to grow to marginally higher amplitudes. $\quad \ldots \ldots \ldots . \ldots \ldots 33$

5.11 Film profile for an evaporating "mathematical" liquid film in zero gravity. $E=0.0001, G=0.0, S=100, M=35.1, \operatorname{Pr}=7.02$. The amplitude of perturbation is changed from $15 \%$ of the initial film thickness $h_{0}\left(1.15 h_{0}\right)$ to $1.50 h_{0}$. Domain size, $L=\lambda_{\max }$. "Cosine squared" initial condition is used. . . . . . . . . . . . . . . 35

5.12 Film profile for an evaporating mathematical liquid film in zero gravity. $E=0.0001, G=0.0, S=100, M=35.1, \operatorname{Pr}=7.02$. The amplitude of perturbation is $5 \%$ of initial film thickness. Domain size, $L=\lambda_{\max }$, $2 \lambda_{\max }, 3 \lambda_{\max }$. Cosine initial condition is used. . . . . . . . . . . 37

5.13 Film profile for an evaporating mathematical liquid film in zero gravity. $E=0.0001, G=0.0, S=100, M=35.1, \operatorname{Pr}=7.02$. The amplitude of perturbation is $5 \%$ of initial film thickness. Domain size, $L=\lambda_{\max }$, $2 \lambda_{\max }, 3 \lambda_{\max }$. Cosine squared initial condition is used. . . . . . . . . . 37

5.14 Cascade of superharmonics for the cosine-squared initial condition as seen in the DFT plots at rupture . . . . . . . . . . . . . . 38

5.15 Zero gravity evaporation of $2.35 \mathrm{~mm}$ thick DCM. Figure on the left is the cosine initial condition used to perturb the DCM film. Figure on the right shows long-wave destabilized liquid film. . . . . . . . . . . . 40 
5.16 Terrestrial gravity evaporation of $2.35 \mathrm{~mm}$ thick DCM. Figure on the left is the cosine initial condition used to perturb the DCM film. Figure on the right shows lack of long-wave instabilities. . . . . . . . . . . . 40

5.17 Zero gravity evaporation of $2.35 \mathrm{~mm}$ thick DCM with a uniform random perturbation in a domain whose side is a non-whole number multiple of fastest growing wavelength. Figure on the left is the cosine initial condition used to perturb the DCM film. Figure on the right shows long-wave destabilized liquid film. . . . . . . . . . . . . . . . 41

5.18 Zero gravity evaporation of $2.35 \mathrm{~mm}$ thick DCM with a uniform random perturbation in a domain whose length and breadth are a nonwhole number multiple of fastest growing wavelength . . . . . . . . . 42

5.19 Rupture in 3D case . . . . . . . . . . . . . . . . . . 43

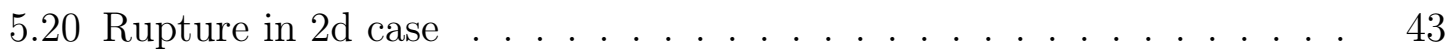

5.21 Rupture in 3D case at $T=1319.0$. . . . . . . . . . . . . . . . . . . . 43

5.22 default $\ldots \ldots \ldots \ldots \ldots$. . . . . . . . . . . . . . . . . . . . . 43

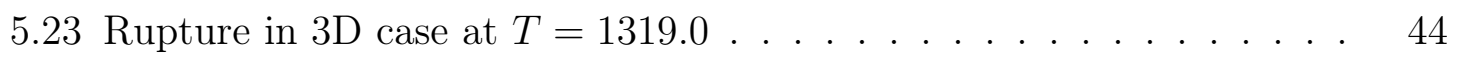

5.24 Rupture in 2D simulation. . . . . . . . . . . . . . . . . . . . 44

5.25 Thick line: Slowly evaporating mathematical fluid $(\mathrm{E}=0.0001)$ with periodic boundary condition at rupture. Dashed line: free boundary conditions at the same time free boundary conditions. $L=\lambda_{\max }$. . . 46

5.26 Thick line: Slowly evaporating mathematical fluid $(\mathrm{E}=0.0001)$ with periodic boundary condition at rupture. Dashed line: free boundary condition at the same time with free boundary conditions. $L=2 \lambda_{\max } \quad 46$

5.27 Thick line: Slowly evaporating mathematical fluid $(\mathrm{E}=0.0001)$ with periodic boundary condition at rupture. Dashed line: free boundary conditions at the same time with free boundary conditions. $L=3 \lambda_{\max } \quad 46$

5.28 Thick line: Slowly evaporating mathematical fluid $(\mathrm{E}=0.0001)$ without the buoyancy term. Dashed line: Full evolution equation with $R=10^{-4} . L=\lambda_{\max } \cdot T_{\text {Rup, without Ra }}=1781, T_{\text {Rup, with Ra }}=1607 .$. . . 47 


\section{LIST OF TABLES}

1.1 Important properties and scales. . . . . . . . . . . . . . . 6

1.2 Important non-dimensional numbers. . . . . . . . . . . . . . . . . . . 7

$5.1 \quad$ Effect of vapor recoil on rupture time and amplitude per Figure $|5.10| \square \quad 32$

5.2 Surface tension term . . . . . . . . . . . . . . . . . 35

5.3 Thermocapillarity term . . . . . . . . . . . . . . . . . . 35

5.4 Comparison of surface tension(ST) $\nabla \cdot\left(h^{3} \nabla \nabla^{2} h\right)$ and thermocapillarity

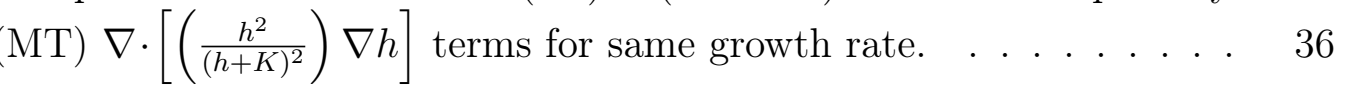

5.5 Comparison of non-dimensional numbers. . . . . . . . . . . . . . . . 39 


\section{ABSTRACT}

Liquid films, evaporating or non-evaporating, are ubiquitous in nature and technology. The dynamics of evaporating liquid films is a study applicable in several industries such as water recovery, heat exchangers, crystal growth, drug design etc. The theory describing the dynamics of liquid films crosses several fields such as engineering, mathematics, material science, biophysics and volcanology to name a few.

Interfacial instabilities typically manifest as undulation of an interface from a presumed flat state or by the onset of a secondary flow state from a primary quiescent state or both. To study the instabilities affecting liquid films, an evaporating/nonevaporating Newtonian liquid film is subject to a perturbation. Numerical analysis is conducted on configurations of such liquid films being heated on solid surfaces in order to examine the various stabilizing and destabilizing mechanisms that can cause the formation of different convective structures. These convective structures have implications towards heat transfer that occurs via this process. Certain aspects of this research topic have not received attention, as will be obvious from the literature review.

Static, horizontal liquid films on solid surfaces are examined for their resistance to long wave type instabilities via linear stability analysis, method of normal modes and finite difference methods. The spatiotemporal evolution equation, available in literature, describing the time evolution of a liquid film heated on a solid surface, is utilized to analyze various stabilizing/destabilizing mechanisms affecting evaporating and non-evaporating liquid films. The impact of these mechanisms on the film stability and structure for both buoyant and non-buoyant films will be examined by the variation of mechanical and thermal boundary conditions.

Films evaporating in zero gravity are studied using the evolution equation. It is found that films that are stable to long wave type instabilities in terrestrial gravity are prone to destabilization via long wave instabilities in zero gravity. 


\section{STABility OF Liquid Films}

\subsection{Ubiquity of Liquid Films}

Although it might be surprising that similar mathematical ideologies could be applied to several areas of nature and technology, liquid films and their flows occur over a wide range of length scales [3] and are central to numerous areas of engineering (coatings, falling films), geophysics (lava flows, flow of ice sheets), biophysics (rupture

of tear films) etc. In all these areas of physics, same or similar equations govern the dynamics of said liquid films. The rapid progress of science of liquid films, its applications in the coatings industry, the medical world and microfluidics have received strong attention.

Coatings receive widespread attention in several industries. They are used as lubricants, paint for rust prevention and aesthetics in architecture, in time release capsules and tablets in the medical industry, the paper and pulp industry and the microelectronics fabrication industry. A report from the Brookhaven National Laboratory estimated that in excess of $\$ 100$ billion per year, is spent on the prevention and remediation of rust damage [4.

Besides coatings, liquid films find applications in the food processing industry [5, 6], cooling of server towers that was a $\$ 36$ Billion industry in 2007 [7-9] and for improved oil recovery from petroleum reservoirs [10, 11].

\subsection{Destabilizing and Stabilizing Mechanisms}

Rayleigh-Benard convection and Marangoni-Benard convection structures were considered curious when encountered by Benard [12] and Thomson [13]. Their work subsequently gave rise to the notion of convective structures that affect and impact liquid films heated on solid surfaces with or without a free surface at the top. Early researchers concerned themselves with the origin and nature of convective structures in heated liquid films [13 21].

There are several destabilizing mechanisms that could affect a liquid film. Rupture due to Van der Waals forces, thermocapillarity-driven formation of finger-like structures and subsequent rupture due to thermocapillarity or solutocapillarity ef- 


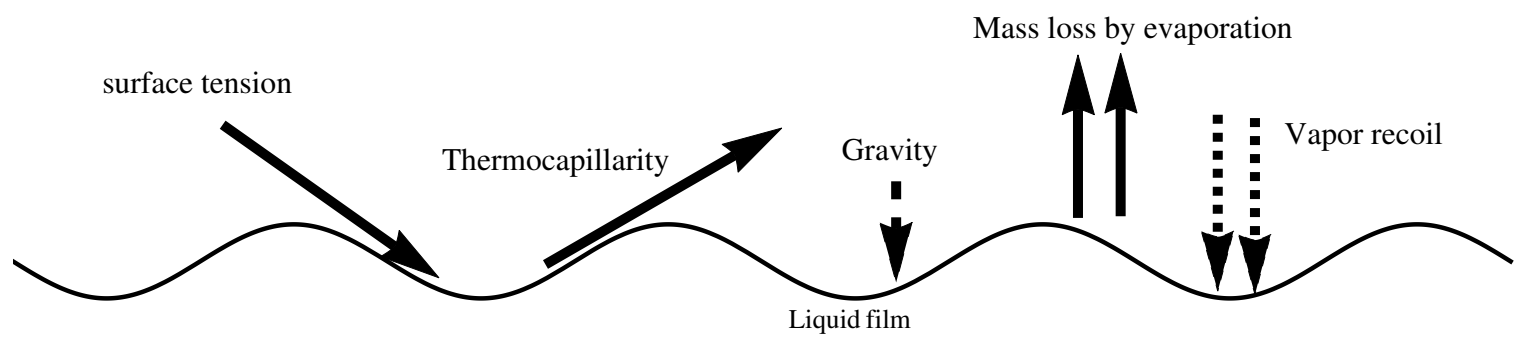

Hot Plate at constant temperature

Figure 1.1. Various stabilizing and destabilizing forces that affect an evaporating liquid film.

fects and formation of pendant drops as a result of Rayleigh-Taylor unstable liquid films are few such destabilizing effects. The various forces acting on the surface of a horizontal, non-draining, evaporating liquid films on a hot solid substrate (substrate is at constant temperature) are depicted in Figure 1.1.

A unified approach to explain the effect of various stabilizing and destabilizing mechanisms via the evolution equation was undertaken by Williams and Davis [22]. They developed a non-dimensional spatiotemporal evolution equation from the governing equations of fluid dynamics and tested it to predict rupture time of a thin film subject to surface tension and Van der Waals interactions alone. A linear stability analysis [23, 24] was also conducted to derive the fastest growing wavelength for such a thin film. The scope of this evolution equation was extended by Burelbach et al. 25] to isolate and examine the effects of mass loss by evaporation and vapor recoil in conjunction with surface tension and Van der Waals interactions. Condensing liquid films have also been studied via this evolution equation [25].

Several encouraging verifications of the long-wave theory and evolution equation versus the experimental results have appeared in the literature. A non-linear analysis was conducted by Sharma and Ruckenstein [26] on non-evaporating liquid films subject to a perturbation. He proved that a non-linear stability analysis produced 
results that were within about $10 \%$ of those produced by the linear stability analysis for a perturbation amplitude of 0.1 times the initial film thickness. It was also concluded that the nonlinearities in the governing equations become important when the amplitude of the initial perturbation gets larger.

Burelbach et al. [27] carried out a series of experiments in an attempt to check the long-wave theory of Tan et al. [28] for steady thermocapillary flows induced by nonuniform heating of the solid substrate. The measured steady shapes were favorably tested against theoretical predictions for layers less than $1 \mathrm{~mm}$ thick under moderate heating conditions. Pradhan and Samal [29], Straughan [30] attempted to study the instability of an inviscid and viscous layers of liquid under the influence of variable gravity which varies with thickness of the liquid layer. A fastest growing wavelength does not exist in the presence of a stabilizing gravity field with no sources of destabilization. The growth rate of instabilities, as calculated via a linear stability analysis, is negative. In other words, a gravity field can have a strong stabilizing effect on the growth of instabilities.

Vanhook et al. [31] performed experiments to examine surface tension-driven Benard convection and compared the results with the evolution equation to find that the experimental occurrence of a rupture inducing instability occurred $35 \%$ earlier than predicted by the evolution equation of Burelbach et al. [25]. The reason for this discrepancy was concluded to be the use of periodic boundary conditions in the simulation. Most material processing occurs in containers with rigid boundaries. Ostrach [32] warrants the use of physical conditions such as pinned or free boundary conditions.

Methods of surface irradiation or internal heat generation to control evaporatively driven instabilities have been examined via a modified evolution equation [33 35] generation. It was concluded that if the strength of the heat source was strong enough, then based on a critical heat flux value the instability and rupture of thin films owing to thermocapillary effect can be hindered and even completely suppressed by heat generation.

There has been some research in treating the evaporating liquid film system as a two layer system instead of a one-sided single layer and the introduction of nonNewtonian liquids over the heated substrate. Yiantsios and Higgins [36] used a viscosity ratio such that $\mu_{v} / \mu_{l}>>1$, where $\mu_{v}$ and $\mu_{l}$ are vapor and liquid viscosities. Non-Newtonian fluids were modeled and via an Arrhenius rate type equation for the fluid viscosity or a power law fluid [37, 38].

The stability of a film governed by the one-sided evolution equation was compared with a two-sided evolution equation that includes a vapor diffusion [39. It was postulated that diffusion limited evaporation has a stabilizing effect on the film dynamics. However, we observe that an incorrect time scale was utilized for the development of this two-sided model by [39]. The viscous time scale utilized for the evolution equa- 
tion by Krishnamoorthy et al. [1], Oron [2], Burelbach et al. 25] was based on the assumption that the evaporation time scale was much larger than the viscous time scale.

The effect of thermal conductivity of the vapor phase was studied by Kliakhandler et al. [40]. The equation for heat transfer through the vapor was retained and the resulting two-sided equation was compared with the one-sided form. The effect of coupling the vapor and liquid regions through the thermal conductivity boundary condition results in the evolution equation diverging from the physical phenomena as the film thickens via condensation. However, for small vapor to liquid thermal conductivity ratios, as is common in most practical cases, the one sided model predicts condensation accurately.

A three dimensional evolution equation was simulated by Oron [2]. The choice of initial conditions and the size of the domain size governs the structure of emerging patterns. Oron [2] compared their results with those of Krishnamoorthy et al. [1] , the latter solving the complete set of governing equations. The results obtained from the evolution equation matched those obtained from the full system of governing equations which modeled a liquid film under the influence of stabilizing gravity, destabilizing thermocapillary forces and stabilizing surface tension.

Burelbach et al. 25] and Williams and Davis [22] explained the effect of various stabilizing and destabilizing mechanisms via a single non-linear evolution equation. This one-sided equation in which vapor and liquid dynamics were decoupled, was based on Benny's treatment of falling films Benny [23, 24]. This equation has been a significant cornerstone for all future numerical analysis of evaporating liquid films. This non-dimensional spatiotemporal evolution equations as derived by Williams and Davis [22] and Burelbach et al. 25] is given in equation 1.1.

$$
h_{t}+\frac{E}{(h+K)}+S\left(h^{3} h_{x x x}\right)_{x}+\left[\left(\frac{A}{h}-G h^{3}+\frac{E^{2}}{D} \frac{h^{3}}{(h+K)^{3}}+\frac{K M}{\operatorname{Pr}} \frac{h^{2}}{(h+K)^{2}}\right) h_{x}\right]_{x}=0
$$

Equation 1.1 is an equation for film thickness, $h(x, t)$, where all other properties such as pressures and velocities are dependent upon the film thickness. For derivation details, the reader is directed to Williams and Davis [22], Burelbach et al. [25]. A subscript denotes partial differentiation. $E(h+K)^{-1}$ represents mass loss by evaporation where $K$ is a measure of volatility of the liquid film. $K=0$ denotes a uniform surface temperature with no vapor recoil or Marangoni effects, while $K^{-1}=0$ denotes a non-volatile liquid film. $S\left(h^{3} h_{x x x}\right)_{x}$ represents the effect of surface tension. $\left(A h^{-1} h_{x}\right)_{x}$ represents the effect of Van der Waals force. $\left(-G h^{3} h_{x}\right)_{x}$ represents the effect of gravity on the interface. $\left[\left(E^{2} D^{-1}(h+K)^{-3} h^{3}\right) h_{x}\right]_{x}$ represents vapor recoil. $\left.\left[K M P^{-1}(h+K)^{-2} h^{2}\right) h_{x}\right]_{x}$ represents the effect of Marangoni effect when the surface has a non-uniform temperature distribution when $0<K<\infty$. Important length 
Table 1.1. Important properties and scales.

$$
\begin{aligned}
& h, \text { film thickness, } h(x, y, z, t) \\
& h_{0} \text {, initial mean film thickness } \\
& L, \text { domain size } \\
& \lambda, \text { wavelength } \\
& \text { q, wavenumber } \\
& \kappa, \text { interface curvature } \\
& \rho, \text { density } \\
& \mu, \text { viscosity } \\
& \nu, \text { kinematic viscosity } \\
& \sigma, \text { surface tension } \\
& \sigma_{T}, d \sigma / d T \\
& \alpha, \text { thermal diffusivity } \\
& k, \text { liquid thermal conductivity } \\
& \tilde{h}, \text { heat transfer coefficient in vapor } \\
& \beta, \text { coefficient of thermal expansion } \\
& \Lambda, \text { latent heat of vaporization } \\
& \Pi, \text { disjoining pressure } \\
& T, \text { temperature } \\
& T^{*},\left(T-T_{\text {sat }}\right) /\left(T_{H}-T_{\text {sat }}\right)
\end{aligned}
$$

scales and fluid properties are listed in Table 1.1 and important non-dimensional terms in the evolution equation are described in the Table 1.2 .

The evolution equation is solved as an initial value problem with periodic boundary conditions and in all cases, a normal mode analysis was performed as a means of defining the convective structures. The method of normal modes has been outlined in several sources [22, 25, 41, 42] and the references therein. 
Table 1.2. Important non-dimensional numbers.

$$
\begin{aligned}
& \text { Evaporation Number, } \mathrm{E} \equiv \frac{k \Delta T}{\rho \nu \Lambda} \\
& \text { Non-Equilibrium Coefficient, } \mathrm{K} \equiv \frac{k T_{\mathrm{sat}}^{3 / 2}}{\alpha h_{0} \rho^{2} \Lambda^{2}}\left(\frac{2 \pi R_{g}}{M_{W}}\right)^{1 / 2} \\
& \text { Surface Tension number, } \mathrm{S} \equiv \frac{\sigma h_{0}}{3 \rho \nu^{2}} \\
& \text { Dimensionless Hamaker Coefficient, } \mathrm{A} \equiv \frac{A^{\prime}}{6 \pi h_{0} \rho \nu^{2}} \\
& \text { Galileo number, } \quad \mathrm{G} \equiv \frac{g h_{0}^{3}}{\nu^{2}} \\
& \text { Marangoni number, } \mathrm{M} \equiv \frac{\sigma_{T} \Delta T h_{0}}{2 \rho \nu k} \\
& \text { Rayleigh number, } \mathrm{Ra} \equiv \frac{g \beta \Delta T h_{0}^{3}}{\nu k} \\
& \text { Dimensionless Latent Heat, } \quad \mathcal{L} \equiv \frac{8 h_{0}^{2} \Lambda}{9 \nu} \\
& \text { Thermocapillary number, } \mathrm{C} \equiv \frac{\sigma_{T}\left(T_{h}-T_{\mathrm{sat}}\right)}{\sigma} \\
& \text { Biot number, } \mathrm{Bi} \equiv \frac{\tilde{h} h_{0}}{k} \\
& \text { modified Biot number, } \mathrm{B} \equiv 1+\mathrm{Bi} \mathrm{K} \\
& \text { Bond number, Bo } \equiv \frac{\mathrm{G}}{\mathrm{S}} \\
& \text { Buoyancy number, } \quad \mathrm{R} \equiv \frac{\mathrm{Ra}}{\operatorname{PrS}} \\
& \text { Prandlt number, } \operatorname{Pr} \equiv \frac{\nu}{\alpha}
\end{aligned}
$$




\section{InCLUSION Boussinesq APPROXIMATION}

A modified evolution equation captures the destabilizing effect of buoyancy by amending the evolution equation originally described by Burelbach et al. [25]. To do this, the Navier-Stokes equations for liquid and vapor side dynamics and the interfacial boundary conditions for mass, momentum, energy and stress balance are non-dimensionalized using the film thickness as length scale and a viscous time scale. The non-dimensionalized equations on the liquid side are decoupled from the vapor side by making observations about property ratios. This yields a "one-sided model". The one sided non-dimensionalized equation is now subject to the long wave approximation, which is similar to the lubrication approximation. The long wave treatment of the one sided model and the interfacial boundary conditions gives a non-linear partial differential equation of fourth order in film thickness. This is the film "evolution equation". All fluid properties such as pressures and temperature fields can be described as functionals of film thickness. Hence, solving the film evolution equation under certain conditions is tantamount to solving the full set of Navier-Stokes equations. The philosophy of the evolution equation is to allow the solution of one equation to describe film dynamics through simple parameter variation.

\subsection{Boussinesq Approximation and Scaling}

While deriving an expression for the modified evolution equation we have included the Boussinesq approximation in the Navier Stokes' equations. The Boussinesq approximation has not been previously included. By including the Boussinesq approximation for density variation in the momentum equation:

$$
\begin{gathered}
\rho_{0}\left[\frac{\partial v}{\partial t}+v \cdot \nabla v\right]=-\nabla P+\mu \nabla^{2} v+\underbrace{\rho_{0}\left[1-\beta\left(T-T_{0}\right) g\right]}_{\text {Boussinesq approximation }} \\
P=p+\rho_{0} g h \\
\frac{\partial T}{\partial t}+v \cdot \nabla T=\alpha \nabla^{2} T
\end{gathered}
$$




$$
\frac{\partial u}{\partial x}+\frac{\partial w}{\partial z}=0
$$

Equations 2.1, 2.3, 2.4 are the momentum, energy and continuity equations respectively for the 2 dimensional problem of a liquid film being heated from underneath by a hot substrate. The film is non-draining, Newtonian and homogeneous.

The equations are scaled using viscous scales for time, velocity and pressure viz., $h_{0}^{2} / \nu, \nu / h_{0}$ and $\rho_{0} \nu^{2} / h_{0}^{2}$ where $\nu$ is the kinematic viscosity. The temperature difference across the film thickness is scaled as $T^{*}=\left(T-T_{\text {sat }} / T_{H}-T_{\text {sat }}\right)$. Here, $T_{H}$ is the temperature of the hot substrate the film rests on. The viscous time scale is assumed to be smaller than the evaporative time scale (evaporation is slow). This scaling yields the following non-dimensional equations:

$$
\begin{gathered}
{\left[\frac{\partial v}{\partial t}+v \cdot \nabla v\right]=-\nabla \wp+\nu \nabla^{2} v} \\
\wp=p+G z-(\operatorname{Ra} / \operatorname{Pr}) \theta(z)
\end{gathered}
$$

The Galileo number, G, is a measure of importance of gravity and seeing as long wave instabilities are damped by gravity. The Rayleigh number, Ra, is the ratio of buoyant effects due to thermal expansion to viscous and thermal dissipation. The Prandtl number, Pr, is the ratio of momentum diffusivity to thermal diffusivity. $\theta(z)$ is the linear temperature profile across the film thickness and is derived assuming that heat transfer is purely conductive.

$$
\begin{gathered}
\operatorname{Pr}\left[\frac{\partial T}{\partial t}+v \cdot \nabla T\right]=\nabla^{2} T \\
\frac{\partial u}{\partial x}+\frac{\partial w}{\partial z}=0
\end{gathered}
$$

Equations 2.5, 2.7, 2.8 are the non-dimensional momentum, energy and continuity equations respectively.

\subsection{Interfacial Jump Conditions and Scaling}

The interfacial jump balance equations can be derived from the general integral form of conservation equations by the application of Leibniz's theorem and Gauss' divergence theorem for the differentiation inside the integral and for expression volume integrals in terms of area integrals respectively.

\subsubsection{Jump Mass Balance}

At the interface $z=h(x, t)$, the jump mass balance expresses the conservation of mass of the evaporating liquid across the interface. The jump mass balance is given 
by:

$$
J=\rho\left(v-v^{I}\right) \cdot \hat{n}=\rho^{v}\left(v^{I}-v^{v}\right) \cdot \hat{n}
$$

Where superscript $I$ terms are properties that occur at the interface and superscript $v$ terms are those that occur in the vapor phase. Terms without a superscript are properties that occur in the liquid phase. $J$ is the mass flux.

\subsubsection{Jump Energy Balance}

The jump energy balance at the liquid vapor interface is given by:

$$
J \Lambda+\frac{J^{3}}{2}\left(\frac{1}{\rho^{v 2}}-\frac{1}{\rho}\right)=k^{v} \nabla T^{v} \cdot \hat{n}-k \nabla T \cdot \hat{n}
$$

The first term on the left side is the latent heat of evaporation that accompanies the mass flux, $J$ by evaporation. The second term represents the difference in kinetic energies in the vapor and liquid phases. The term on the right side describes the difference in heat transfer by pure conduction in the vapor phase and liquid phase. This difference in heat transfer is, hence, balanced by the evaporative latent heat and kinetic energy difference.

\subsubsection{Normal Stress Jump Condition}

$$
J^{2}\left(\frac{1}{\rho^{v}}-\frac{1}{\rho}\right)+\hat{n} \cdot\left(\mathcal{T}-\mathcal{T}^{v}\right) \cdot \hat{n}=\kappa \sigma
$$

$\mathcal{T}$ is the stress tensor which has in it the normal stress (pressure) and the shear stress terms. This is a tensor and hence a dot product with a vector (a normal or tangential component) yields a tractive force with units of force per unit area. For a non-evaporating liquid film, the difference in normal stress across the interface, $\hat{n} \cdot\left(\mathcal{T}-\mathcal{T}^{v}\right) \cdot \hat{n}$ results from the curvature $\kappa$ at the interface. However, for an evaporating liquid film, it is necessary to add the effect of vapor recoil on the troughs and peaks of the deformable interface. Hence the vapor recoil term, $J^{2}\left(1 / \rho^{v}-1 / \rho\right)$, representing the change in momentum is added to the left hand side of the Equation 2.11.

\subsubsection{Shear Stress Jump Condition}

$$
\hat{t} \cdot\left(\mathcal{T}-\mathcal{T}^{v}\right) \cdot \hat{n}=\nabla \sigma \cdot \hat{t}+J\left(v-v^{v}\right) \cdot \hat{n}
$$

The tangential component, $\hat{t} \cdot\left(\mathcal{T}-\mathcal{T}^{v}\right) \cdot \hat{n}$ of the traction force is balanced by the derivative of surface tension that exists along the interface and any discontinuity in tangential velocities, $J\left(v-v^{v}\right) \cdot \hat{n}$. As this discontinuity in tangential velocities is neglected through a no-slip assumption at the interface, the shear stress jump condition is reduced to: 


$$
\hat{t} \cdot\left(\mathcal{T}-\mathcal{T}^{v}\right) \cdot \hat{n}=\nabla \sigma \cdot \hat{t}
$$

\subsubsection{Reducing the Jump Conditions to One-Sided Model}

The jump condition equations 2.10, 2.11 and 2.13 are reduced to a one sided form by observing that the following limits tend to zero:

$$
\begin{aligned}
\frac{\rho^{v}}{\rho} & \rightarrow 0 \\
\frac{\mu^{v}}{\mu} & \rightarrow 0 \\
\frac{k^{v}}{k} & \rightarrow 0
\end{aligned}
$$

Under conditions imposed by these limits, the interfacial conditions take the following form:

$$
\begin{gathered}
\frac{J^{2}}{\rho^{v}}+\hat{n} \cdot \mathcal{T} \cdot \hat{n}=\kappa \sigma \\
\hat{t} \cdot \mathcal{T} \cdot \hat{n}=\nabla \sigma \cdot \hat{t} \\
J \Lambda+\frac{J^{3}}{2 \rho^{v^{2}}}=-k \nabla T \cdot \hat{n}
\end{gathered}
$$

\subsubsection{Scaling Interfacial Jump Conditions}

Scaling (non-dimensionalization) of the governing equations is described in subsection 2.1. The jump condition equations 2.9, 2.14, 2.15 and 2.16 are also scaled (nondimensionalized) using viscous scales for time, velocity and pressure viz., $h_{0}^{2} / \nu, \nu / h_{0}$ and $\rho_{0} \nu^{2} / h_{0}^{2}$ where $\nu$ is the kinematic viscosity. The temperature is scaled as $T^{*}=$ $\left(T-T_{\text {sat }} / T_{H}-T_{\text {sat }}\right)$. The viscous time scale is assumed to be smaller than the evaporative time scale (evaporation is slow). As a result of the scaling, several nondimensional terms appear in the jump conditions. The scaled boundary conditions are as follows:

The jump mass balance at the interface:

$$
E J=\left(v-v^{I}\right) \cdot \hat{n}=\frac{2}{3} D\left(v^{I}-v^{v}\right) \cdot \hat{n}
$$

We term the ratio of evaporative to viscous time scales, the evaporation number, $E=k \Delta T / \rho \nu \Lambda$. The ratio of densities is given by $D=3 \rho^{v} / 2 \rho$.

The scaled energy jump is given by:

$$
J+\left(E^{2} D^{-2} \mathcal{L}^{-1}\right) J^{3}=-\nabla T \cdot \hat{n}
$$


Here $\mathcal{L}=8 h_{0}^{2} \Lambda / 9 \nu^{2}$. This is a measure of latent heat.

The scaled normal stress condition is given by:

$$
-\frac{3}{2} E^{2} D^{-1} J^{2}+p-\hat{n} \cdot(2 \tau) \cdot \hat{n}=3 S(1-C T) \nabla \cdot \hat{n}
$$

The non-dimensional surface tension is defined as $S=\sigma h_{0} / 3 \rho \nu^{2}$ while the capillary number is give by $C=\sigma_{T} \Delta T / \sigma$.

The scaled shear stress condition is given by:

$$
\hat{n} \cdot \tau \cdot \hat{t}=-M \operatorname{Pr}^{-1} \nabla T \cdot \hat{t}
$$

\subsection{Rescaling}

Since long wave instabilities are being examined, the equations are rescaled using a long wavelength, $\lambda$ or a small wavenumber, $\mathrm{k}$ where the wavenumber is defined as, $\mathrm{q}=2 \pi / \lambda$. Various parameters such as spatial coordinates $(x, y, z, X, Y, Z)$, time $(t, T)$, velocity $(u, v, w)$, pressure $(\wp)$, temperature $(T)$, mass flux $(J)$ are rescaled by expanding them in a power series of the wavenumber, $\mathrm{k}$. The rationale behind this expansion is that length, velocity, pressure and temperatures in one direction are significant as compared to the other. For instance, the $x$-velocity, $u$ is greater than the $z$-velocity $w$. The long wave expansion reflects this choice.

$$
\begin{aligned}
X & =\mathrm{q} x \\
Z & =z \\
T & =\mathrm{q} t \\
u & =u_{0}+\mathrm{q} u_{1}+\cdots \\
w & =\mathrm{q}\left(w_{0}+\mathrm{q} w_{1}+\cdots\right) \\
J & =J_{0}+\mathrm{q} J_{1}+\cdots \\
T & =T_{0}+\mathrm{q} T_{1}+\cdots \\
\wp & =\mathrm{q}^{-1}\left(\wp_{0}+\mathrm{q} \wp_{1}+\cdots\right)
\end{aligned}
$$

We substitute the equations 2.21 into the governing equations and the boundary conditions and collect terms at leading order in q. At leading order in $q$ the governing equations become:

$$
\begin{array}{r}
-\wp_{X}+u_{Z Z}=0 \\
-\wp_{Z}=0 \\
u_{X}+w_{Z}=0
\end{array}
$$


We drop the subscript 0 that signifies leading order terms for convenience. Subscripts signify partial derivatives. The boundary conditions at the solid heated substrate, $Z=0$ and at the interface, $Z=h(X, T)$. At $Z=0, u=w=0$ and $T=1$. At $Z=h(X, T)$, evaporation occurs:

$$
\begin{gathered}
h_{T}+\frac{\partial}{\partial X} \int_{a}^{z=h} u \mathrm{~d} z=-E J \\
J=-T_{Z} \\
\wp=\frac{3}{2} E^{2} D^{-1} J^{2}-3 S h_{X X} \\
u_{Z}=-2 M \operatorname{Pr}^{-1}\left(T_{X}+T_{Z} h_{X}\right) \\
K J=T
\end{gathered}
$$

The measure of non-equilibrium at the interface is $K$. It is derived from kinetic theory [18]. When $K \rightarrow 0$ the film is highly volatile as signified with a large mass flux, $J$. If $K$ is large, the film is non-volatile. The scaled constitutive relationship that expresses the mass flux as a function of temperature difference is given by:

$$
K J=T,
$$

where

$$
K=\frac{k T_{s a t}^{3 / 2}}{\hat{\sigma} h_{0} \rho^{v} \Lambda^{2}}\left(\frac{2 \pi R_{g}}{M_{w}}\right)^{1 / 2}
$$

In this constitutive relationship, $\hat{\sigma}$ is the accommodation coefficient, $R_{g}$ is the universal gas constant and $M_{w}$ is the molecular weight.

From equations 2.26 and 2.29, the temperature field and mass flux are expressed in terms of film thickness, $h$, and the non-equilibrium parameter at the interface:

$$
\begin{gathered}
T=1-(h+K)^{-1} Z \\
J=(h+K)^{-1}
\end{gathered}
$$

For the temperature field $\theta(z)$ in the buoyancy term,

$$
\theta(z)=\int^{h}(X, T)_{0}\left[1-(h+K)^{-1} Z\right] d z
$$

From the rescaled momentum Equation 2.22.

$$
u=\wp_{X} \frac{Z^{2}}{2}+c_{1} Z+c_{2}
$$


where $c_{1}$ and $c_{2}$ are constants of integration. By applying no slip at the substrate, $c_{2}=0$. Hence,

$$
u=\wp_{X} \frac{Z^{2}}{2}+c_{1} Z
$$

Substituting the equations for velocity, $u$ and temperature $T$ in the Marangoni condition at the interface as described in Equation 2.28.

$$
u_{Z}=-2 M \operatorname{Pr}^{-1}\left(T_{X}+T_{Z} h_{X}\right)
$$

or

$$
\begin{gathered}
\wp_{X}+c_{1}=-2 M \operatorname{Pr}^{-1}\left(T_{X}+T_{Z} h_{X}\right) \\
c_{1}=-2 M \operatorname{Pr}^{-1}\left[\frac{h h_{X}}{(h+K)^{2}}-\frac{h_{X}}{(h+K)}\right]
\end{gathered}
$$

therefore

$$
u=\wp_{X}\left(\frac{Z^{2}}{2}-h Z\right)+\left[-2 M \operatorname{Pr}^{-1}\left\{\frac{h h_{X}}{(h+K)^{2}}-\frac{h_{X}}{(h+K)}\right\}\right] Z .
$$

The mass loss condition at the interface, Equation 2.25 dictates:

$$
h_{T}+\frac{E}{(h+K)} \frac{\partial}{\partial X} \int_{a}^{z=h} u \mathrm{~d} z=0
$$

$E$ is the evaporation number and is a measure of the strength of evaporation. A high evaporation number suggests a high rate of evaporation. It is a ratio of viscous to evaporative time scales (high rate of evaporation is analogous to low evaporative time scales). Substituting for $u$ in Equation 2.41 gives us the modified evolution equation 2.42 for two-dimensional films whose film thickness varies is a function $h(X, T)$ or equation for three dimensional films whose film thickness is a function $h(X, Y, T)$ :

$$
\begin{aligned}
& h_{T}+\frac{E}{(h+K)}+S\left(h^{3} h_{X X X}\right)_{X}-\frac{G}{3}\left(h^{3} h_{X}\right)_{X}+E^{2} D^{-1}\left[\frac{h^{3} h_{X}}{(h+K)^{3}}\right]_{X}+ \\
& \operatorname{KMPr}^{-1}\left[\frac{h^{2} h_{X}}{(h+K)^{2}}\right]_{X}+\underbrace{\frac{5 R a}{48 \operatorname{Pr}}\left[\frac{K^{2}}{(h+K)^{2}} h^{4}+h^{4}\right] h_{X}}_{\text {buoyancy driven instabilities }}=0
\end{aligned}
$$

A term-by-term description of the evolution equation, in three-dimensions, is given 
in Equation 2.43:

$$
\begin{aligned}
& h_{T}+\underbrace{S \nabla \cdot\left(h^{3} \nabla \nabla^{2} h\right)}_{\text {Surface tension }}-\underbrace{\frac{G}{3} \nabla \cdot\left(h^{3} \nabla h\right)}_{\text {gravity }} \\
& +\underbrace{\nabla \cdot\left[\left(E^{2} D^{-1} \frac{h^{3}}{(h+K)^{3}}+K M \operatorname{Pr}^{-1} \frac{h^{2}}{(h+K)^{2}}\right) \nabla h\right]}_{\text {Vapor recoil and thermocapillarity }} \\
& \underbrace{\frac{5 R a}{48 \operatorname{Pr}}\left[\frac{K^{2}}{(h+K)^{2}} h^{4}+h^{4}\right] \nabla h}_{\text {Buoyancy }}+\underbrace{\frac{E}{h+K}}_{\text {Evaporation }}=0
\end{aligned}
$$

\subsection{Alternative Formulations for Non-Evaporating Films}

Oron et al. [43] used a slightly different formulation to couple the liquid and vapor side at the interface by prescribing a heat flux condition at the interface as against Burelbach et al. [25], who prescribed a mass flux constitutive relationship at the interface.

Oron et al. [43] heat flux condition can be described by the equality of conduction and convection at the interface. Bi, although described as the Biot number in Equation 2.44, is actually the Nusselt number as defined for convective heat transfer. In Equation 2.44, $\theta=1-(\mathrm{Bi} z /(1+\mathrm{Bi} h))$ [43].

$$
\frac{\partial \theta}{\partial z}-\operatorname{Bi} \theta=0
$$

Oron's formalism gives rise to an evolution equation that is slightly different from that proposed by Burelbach. It is depicted in Equation 2.45. The evaporative mass flux and vapor recoil terms have been omitted since this is a non-evaporating liquid film.

$$
h_{T}+S\left(h^{3} h_{X X X}\right)_{X}-\frac{G}{3}\left(h^{3} h_{X}\right)_{X}+\operatorname{Bi} M \operatorname{Pr}^{-1}\left[\frac{h^{2} h_{X}}{(\operatorname{Bi} h+1)^{2}}\right]_{X}=0
$$

A different form of the evolution equation was proposed by Krishnamoorthy et al. [1] for non-evaporating liquid films. In this case, a modified Biot number, $B$, was defined as $B=1+\mathrm{Bi} K$ where $\mathrm{Bi}$ was the Biot number (Nusselt number) as defined by Oron et al. 22 and $K$ is the non-equilibrium coefficient of Krishnamoorthy et al. [1]. Krishnamoorthy et al. [1]'s evolution equation is given in Equation 2.46. 


$$
h_{T}+S\left(h^{3} h_{X X X}\right)_{X}-\frac{G}{3}\left(h^{3} h_{X}\right)_{X}+B M \operatorname{Pr}^{-1}\left[\frac{h^{2} h_{X}}{(B h+1)^{2}}\right]_{X}=0
$$

When $K=0, \mathrm{Bi}=1$ we have in Equation 2.46, $B=1$. The limitation of the evolution equation prescribed by Krishnamoorthy et al. [1] or that by Oron [2] is that it can only capture convective instabilities affecting the interface when the Biot number, $B=1$ for non-evaporating cases. We propose a form of the evolution equation as given in Equation 2.47.

$$
\begin{aligned}
& h_{T}+S \nabla \cdot\left(h^{3} \nabla \nabla^{2} h\right)-\frac{G}{3} \nabla \cdot\left(h^{3} \nabla h\right)+ \\
& \nabla \cdot\left[\left(E^{2} D^{-1} \frac{h^{3}}{\left(\eta_{1} h+\eta_{2}\right)^{3}}+\eta_{1} \eta_{2} M \operatorname{Pr}^{-1} \frac{h^{2}}{\left.\eta_{1} h+\eta_{2}\right)^{2}}\right) \nabla h\right]+ \\
& \underbrace{\frac{5 R a}{48 \operatorname{Pr}}\left[\frac{\eta_{2}^{2}}{\left(\eta_{1} h+\eta_{2}\right)^{2}} h^{4}+h^{4}\right] \nabla h}_{\text {Buoyancy }}+\underbrace{\frac{E}{\eta_{1} h+\eta_{2}}}_{\text {Evaporation }}=0
\end{aligned}
$$

In Equation 2.47, $\eta_{1}=\mathrm{Bi}$ and $\eta_{2}=1$ for non-evaporating liquid films (which have $E=0$ ) and $\eta_{1}=1$ and $\eta_{2}=K$ for evaporating liquid films (which have a non-zero $E)$. 


\section{MaXimizing WaVelength}

A linear stability analysis is performed for the evolution equation as per Oron [2]. The "modified evolution equation" is repeated here for convenience:

$$
\begin{array}{r}
h_{t}+E(h+K)^{-1}+S\left(h^{3} h_{x x x}\right)_{x}+\left(\left(A h^{-1}-G h^{3}+E^{2} D^{-1}(h+K)^{-3} h^{3}+\right.\right. \\
\left.\left.K M \operatorname{Pr}^{-1}(h+K)^{-2} h^{2}\right) h_{x}\right)_{x}+\frac{5 R a}{48 \operatorname{Pr}}\left[\frac{K^{2}}{(h+K)^{2}} h^{4}+h^{4}\right] h_{x}=0
\end{array}
$$

Normalizing the time with the surface tension number, $T=t / S$, we have equation 3.2 of the modified evolution equation from 3.1. This normalization reflects the role that surface tension plays in film stabilization. This normalization leads to destabilizing mechanisms being compared with the stabilizing mechanism of surface tension.

$$
\begin{array}{r}
h_{T}+\epsilon(h+K)^{-1}+\left(h^{3} h_{x x x}\right)_{x}+\left(\left(-B o h^{3}+\delta(h+K)^{-3} h^{3}+\right.\right. \\
\left.\left.m(h+K)^{-2} h^{2}\right) h_{x}\right)_{x}+R\left[\frac{K^{2}}{(h+K)^{2}}+1\right] h^{4} h_{x}=0
\end{array}
$$

Here, $\tau=t / S, B o=G / S, \epsilon=E / S, \delta=E^{2} / D S, m=M K / \operatorname{Pr} S, R=5 R a / 48 \operatorname{Pr} S$.

To obtain the fastest growing (maximizing) wavelength from equation 3.2 , we perturb the base state by a small amount as per linear stability. The base state is a thinning, static layer independent of $X$ and purely a linear function of time $T$.

$$
h=\bar{h}+H(T) \exp ^{i q X},
$$

where $q$ is the wavenumber defined as $q=2 \pi / \lambda$ (where $\lambda$ is the wavelength of perturbation) and $H(T)$ is a small perturbation, such that the time dependent base state $\bar{h} \ggg H(T)$. It is necessary to involve the base state because in case of an evaporating liquid film, as the film thins, the maximizing wavenumber could be a function of the current film thickness. 
For convenience, $\theta=H(T) \exp ^{i q X}$. Term-by-term evaluation of the evolution equation reveals:

$$
\begin{gathered}
\left(\bar{h}_{T}+\theta_{T}\right)+\epsilon\left[\frac{1}{\bar{h}+K 1}-\frac{\theta}{(\bar{h}+K 1)^{2}}\right]+ \\
\left(\bar{h}^{3}+3 \bar{h}^{2} \theta+3 \bar{h} \theta+\theta^{3}\right) q^{4} \theta+B o\left(\bar{h}^{2}+2 \bar{h} \theta+\theta^{2}\right)+ \\
-m\left(\bar{h}^{2}+2 \bar{h} \theta+\theta^{2}\right) q^{2} \theta(h+K)^{-2}-\delta\left(\bar{h}^{3}+3 \bar{h}^{2} \theta+3 \bar{h} \theta^{2}+\theta^{3}\right) q^{2} \theta(h+K)^{-3}+ \\
R\left[\frac{K^{2}}{(h+K)^{2}}(\bar{h}+\theta)^{4} q^{2} \theta-(\bar{h}+\theta)^{4} q^{2} \theta\right]=0
\end{gathered}
$$

Solving for the base state, we have $\bar{h}_{T}=-\epsilon /(h+K)$. Expanding the evaporation term of equation 3.4 and retaining terms purely in $\bar{h}$ and $\theta$ and their higher powers alone, we have

$$
\begin{aligned}
& \left(\frac{-\epsilon}{h+K}+\theta_{T}\right)+\frac{\epsilon}{h+K}-\frac{\theta \epsilon}{(\bar{h}+K)^{2}}+ \\
& \bar{h}^{3} \mathrm{q}^{4} \theta+B o \bar{h}^{2} \mathrm{q}^{2} \theta-m \bar{h}^{2}(\bar{h}+K)^{-2} \mathrm{q}^{2} \theta-\delta \mathrm{h}^{3} \mathrm{q}^{3} \theta(h+K)^{-3}=0
\end{aligned}
$$

Dividing throughout by $\theta$, we have an equation for the growth rate of instabilities, $\omega$, equation 3.6

$$
\begin{aligned}
& \frac{\theta_{T}}{\theta}=\omega=\frac{\epsilon}{(\bar{h}+K)^{2}}-\bar{h}^{3} q^{4}- \\
& B o \bar{h}^{2} q^{2}+m \bar{h}^{2}(\bar{h}+K)^{-2} q^{2}+\delta \bar{h}^{3} q^{2}(\bar{h}+K)^{-3}+ \\
& R\left[\frac{K^{2}}{(h+K)^{2}}(\bar{h}+\theta)^{4} q^{2}-(\bar{h}+\theta)^{4} q^{2}\right]=0
\end{aligned}
$$

The basic mathematical premise of the fastest growing wavelength/wavenumber is that it maximizes the growth rate of instabilities. Hence, finding the maximum of the growth rate gives us the fastest growing wavenumber in this case, since the disturbance is expressed in terms of the wavenumber.

$$
\mathrm{q}_{\max }=\frac{1}{\sqrt{2}}\left[-B o+\delta(h+K)^{-3}+m h^{-1}(h+K)^{-2}+R h\left(\frac{K}{(h+K)^{2}-1}\right)\right]^{1 / 2}
$$

Here, $\delta=2 E^{2} / 3 D S, m=M K / \operatorname{Pr} S, \epsilon=E / S$ and $\bar{h}=-K+\sqrt{(K+1)^{2}-2 \epsilon t}$, and $R=R a / \operatorname{Pr} S . \bar{h}$ is the basic $x$-independent state and is a thinning layer. 


\subsection{Validity of Maximizing Wavenumber}

For an isothermal non-evaporating liquid film $(M=0=E)$ under the effect of surface tension $(\mathrm{S})$ and gravity $(\mathrm{G})$, the maximizing wavenumber, Equation 3.7 reduces to

$$
\mathrm{q}_{\max }=\frac{1}{\sqrt{2}}
$$

This is the same maximizing wavenumber obtained by Burelbach et al. [25, Davis 44. for an isothermal liquid film and hence, the maximizing wavenumber obtained in Equation 3.7 holds good.

When compared with results published in Oron [2] we arrive at the same maximizing wavenumber of 0.068 . In the Chapter 5. Results, all plots contain the "maximizing wavelength" instead of "maximizing wavenumber". The maximizing wavelength is $2 \pi / q_{\max }$.

\subsection{Rescaling to Introduce Bond Number}

One of the simplest reduced cases of the evolution equation is that which depicts an isothermal thin film - a non-evaporating liquid film whose interface is under the influence of Van der Waals forces and surface tension forces alone.

$$
h_{t}+A\left(h^{-1} h_{x}\right)_{x}+S\left(h^{3} h_{x x x}\right)_{x}=0
$$

By introducing rescaling space and time coordinates, $X=(A / S)^{1 / 2} x$ and $T=$ $\left(A^{2} / S\right) t$, respectively, we obtain:

$$
h_{T}+\left(h^{-1} h_{X}\right)_{X}+\left(h^{3} h_{X X X}\right)_{X}=0
$$

The physical lengths are obtained by multiplying $X$ with $h_{0}(A / S)^{-1 / 2}$ and the physical time is obtained by multiplying $T$ by $\left(h_{0}^{2} / \nu\right)\left(S / A^{2}\right)$.

In a more complex case as in Oron [2], where the non evaporating liquid film also has Marangoni stresses on the interface, the rescaling parameters are, $X=x / S$ and $T=t / S$. This yields a rescaled evolution equation, Equation 3.11. The rescaling is different from that utilized in Burelbach et al. [25], which would yield an infinite maximizing wavenumber for zero-gravity situations and their evolution equation could not be used to simulate evaporating liquid films in zero gravity.

$$
h_{T}+\left(h^{3} h_{X X X}\right)_{X}-B o\left(h^{3} h_{X}\right)_{X}+m\left[\left(\frac{h}{K+h}\right)^{2} h_{X}\right]_{X}=0
$$


The physical length is obtained by multiplying $X$ with $h_{0} S$ and the physical time is obtained by multiplying $T$ with $\left(h_{0}^{2} / \nu\right) S$. The film thickness is expressed as a combination of a time dependant base state and a perturbation, $h(X, T)=$ $h \overline{(T)}+H(T) e^{i \mathrm{q} X}$. The derivation of the maximizing wavenumber, in this case, is outlined in Oron [2] and involves several other terms such as the Bond number, Bo and $m$, a non-dimensional number that captures the Marangoni effect (in Equation 3.7).

The advantage of rescaling the length and time scales only with the surface tension number, $S$, instead of both the surface tension number and the Galileo number, $G$ is that zero gravity cases can be studied. When both $S$ and $G$ are involved, the fastest growing wavelength would show an inverse dependence on $G$. For zero gravity cases, when $G=0$, this would lead to an inconvenient singularity. 


\section{Validation And Preliminary Results}

The modified evolution equation is solved as an initial value problem with periodic boundary conditions. The equation poses a challenge since it is considerably stiff in nature. This needs the use of a stiff solver and most authors in literature have chosen to use the backward Euler method. In this dissertation the LSODE (Livermore solver [45]) is applied in conjunction with the backward Euler method since the LSODE method is particularly suited for higher order non-linear equations that are stiff. The Mathematica [46] environment is used to implement the solver. The results obtained are compared with the three-dimensional finite difference simulations of Oron 2 and the two-dimensional CFD results of Krishnamoorthy et al. [1].

As per the film conditions prescribed in Krishnamoorthy et al. [1] 2D simulations, the liquid film is under the effect of stabilizing surface tension $(S=100)$, stabilizing gravity $(G=1 / 3)$ and a destabilizing effect of thermocapillarity modeled via the Marangoni number $(M=35.1)$. The Prandt number, Pr, is set to 7.02. These value of non-dimensional parameters are henceforth referred to as the properties of the "mathematical fluid". These non-dimensional fluid parameters were so chosen to destabilize the film to the greatest extent (maximum possible growth rate of instabilities). These non-dimensional fluid parameters do not represent the properties of any physical fluid. However, a low centistoke silicone oil has fluid parameters close [4] to those chosen for our validation. From investigation, it was ascertained that these fluid properties are merely academic and used by the authors to make a strong point about destabilizing/stabilizing mechanisms that affect liquid films.

Formation of thermocapillary fingers and eventual rupture from a wavy, cosine initial condition $\left(1-0.05 \cos q_{\max } x\right)$ are plotted in Figures $4.1,4.2,4.3$. These results are within about $1 \%$ of those published by Krishnamoorthy et al. [1] who used CFD to simulate a non-evaporating liquid film subject to destabilizing thermocapillarity and stabilizing surface tension and gravity.

As further validation, 3D simulations were performed with the evolution equation. The results obtained compared favorably with the $3 \mathrm{D}$ results published by Oron [2]. In Oron's studies [2], the three-dimensional liquid film was under the influence of stabilizing surface tension $(S=100)$, stabilizing gravity $(G=1 / 3)$ and destabilizing thermocapillarity with $(M=35.1)$. 
The results obtained by solving the evolution equation in a three-dimensional domain are depicted via profile and contour plots in Figures 4.4 and 4.5. This depicts the rupture profile of the liquid film when subject to a wavelength twice (Figure 4.4) or four times (Figure 4.5) the fastest growing one.

\subsection{Secondary Structures}

In the study of thermocapillarity in thin liquid films, secondary structures are formed as a result of thermocapillarity (Marangoni effect). Yeo et al. [48] observed secondary structures and conjectured that the amplitude of these secondary structure ("fractal like structure" as they describe these structures) was dependant on the Biot number (heat transfer condition at interface). The occurence of secondary structures was governed by the balance between stabilizing surface tension and destabilizing thermocapillarity. Joo et al. [49] reports that the thinning of a liquid film due to thermocapillarity gives rise to higher harmonics from a mass balance standpoint. Boos and Thess [50] explained the film rupture and formation of secondary structures from the viewpoint of the temperature dependence of surface tension. Liquid mass is driven to regions of higher surface tension (cooler regions further away from the hot plate). This leads to finger-like thermocapillary structures. They also explain that large surface tension would work against the formation of secondary structures while large temperature differences enhanced secondary structure formation.

\subsection{Definition of Rupture}

Rupture time physically corresponds to when the film ruptures by exposing the substrate as a cumulative response to thermocapillarity, mass loss by evaporation, concurrent vapor recoil and disjoining pressures. The rupture time quoted in numerical simulations, be they CFD based or based on long wave theory, are seldom the actual "rupture time" experienced by a liquid film. Computation results are commonly terminated when the interface shape cannot be resolved with sufficiently large fourier modes [1, 2]. Yeo et al. [48] halted their simulations when the film profile was stiff and the simulation could not be continued with sufficient accuracy even with stiff solvers. We define the rupture time as that time step when the solver reports a stiff set of equations that cannot be resolved further with good accuracy even though the appropriate ODE intergration method (LSODE, $12^{\text {th }}$ order is used). Coincidentally, we find that equation stiffness occurs when the film thickness is around $5 \%$ of it's initial thickness $h_{0}$. When the physical times and wavelengths were calculated for a real liquid (Dichloromethane/DCM in this case), we find that for an initially $2.35 \mathrm{~mm}$ thick DCM film in zero gravity, stiffness occurs when the film is about $115 \mu \mathrm{m}$ thick. 


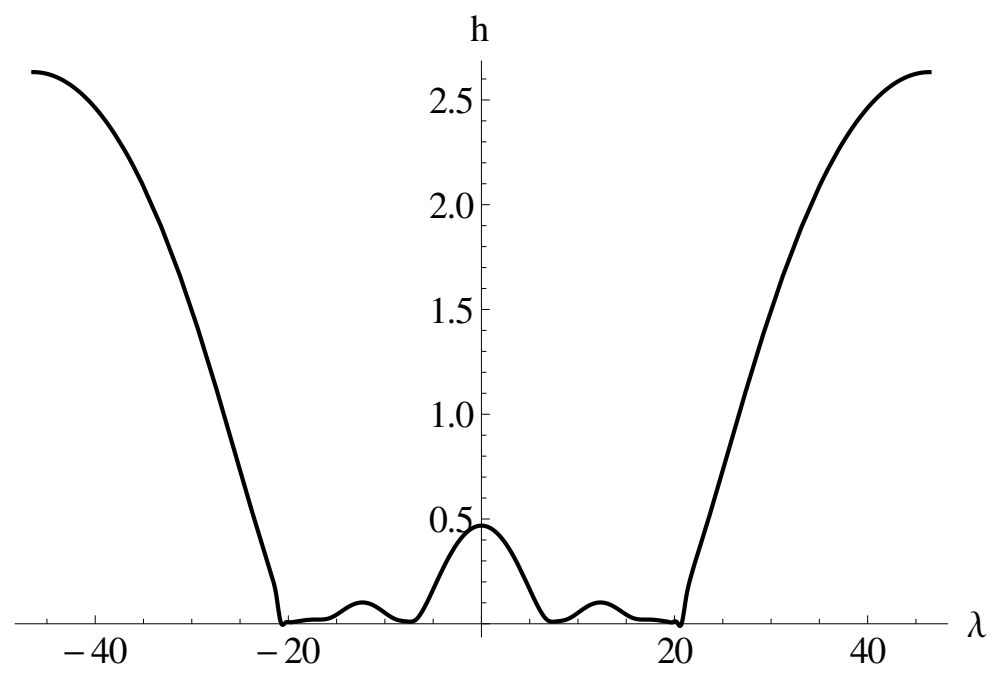

Figure 4.1. When $\lambda=\lambda_{\max }$ and $\mathrm{M}=35.1, \operatorname{Pr}=7.20, \mathrm{~S}=100, \mathrm{G}=1 / 3$, rupture takes place at $\mathrm{t}=1280.0$ (within $1 \%$ of $\mathrm{CFD}$ results [1]).

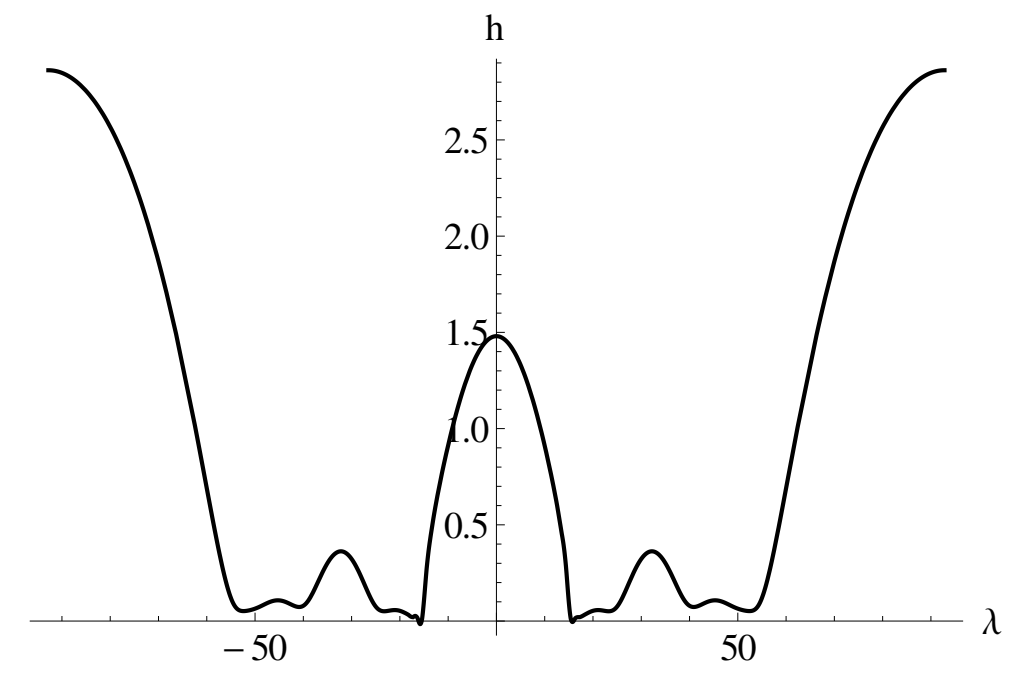

Figure 4.2. When $\lambda=2 \lambda_{\max }$ and $\mathrm{M}=35.1, \mathrm{Pr}=7.20, \mathrm{~S}=100, \mathrm{G}=1 / 3$, rupture takes place at $\mathrm{t}=2475.0$ (within $1 \%$ of $\mathrm{CFD}$ results [1]). 


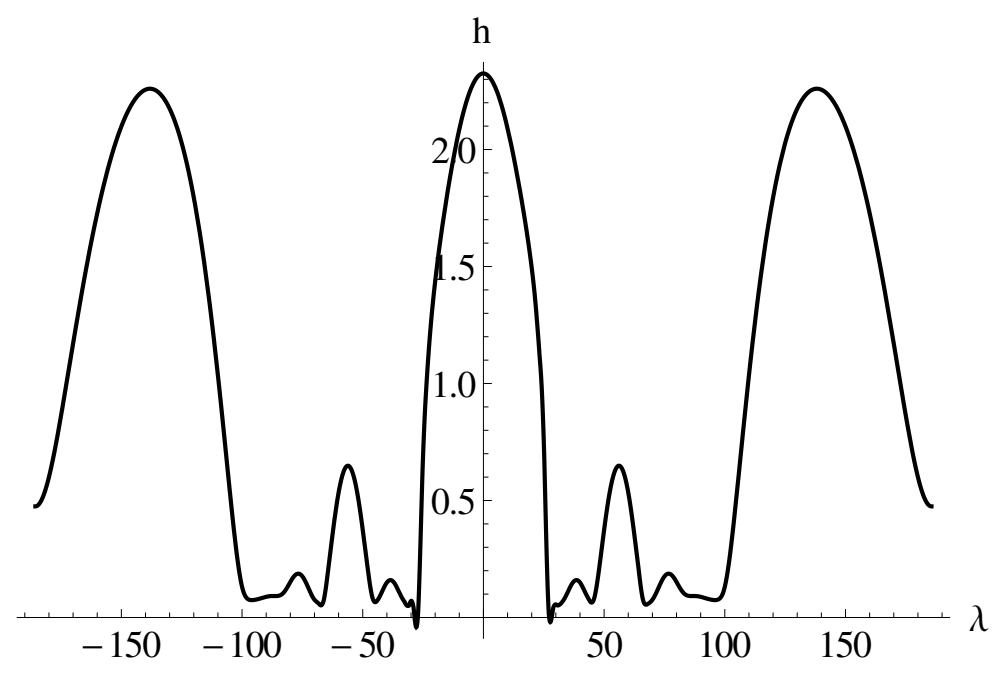

Figure 4.3. When $\lambda=4 \lambda_{\max }$ and $\mathrm{M}=35.1, \operatorname{Pr}=7.20, \mathrm{~S}=100, \mathrm{G}=1 / 3$, rupture takes place at $\mathrm{t}=4613.0$ (within $1 \%$ error of $\mathrm{CFD}$ results [1]). 

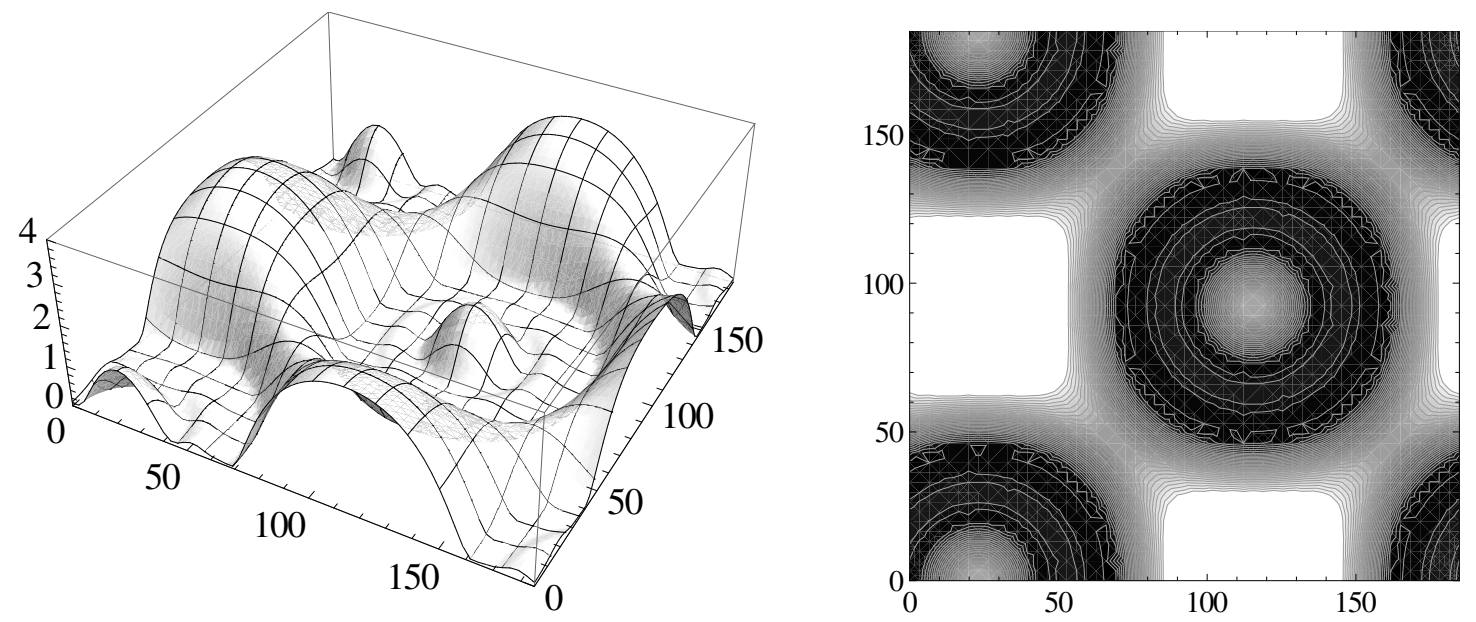

Figure 4.4. Non-evaporating liquid film under the effect of surface tension $(S=100)$, gravity, $(G=1 / 3)$ and thermocapillarity $(M=35.1)$ as in Oron [2]. With $\lambda=2 \lambda_{\max }$, the rupture time is revealed to be 2023.0. This is within $5 \%$ of that published by Oron et al. The contour plot is qualitatively the same as that of Oron [2] and is depicted here merely for a full comparison with Oron's results.
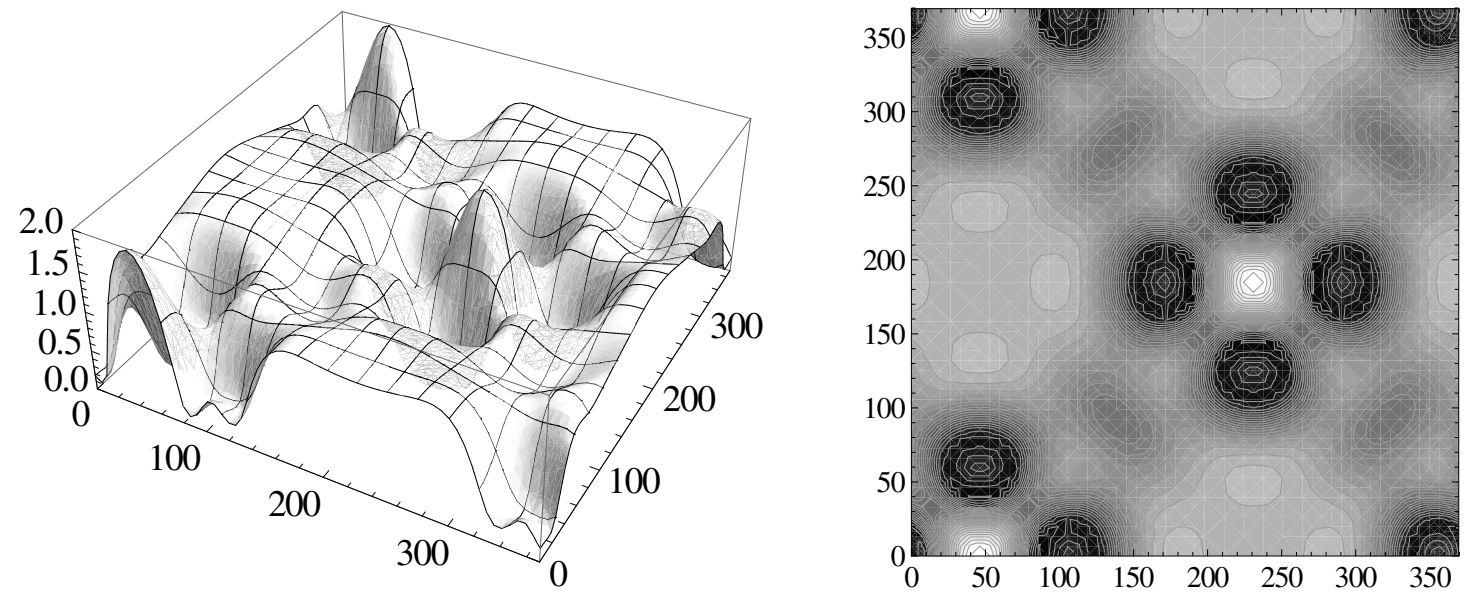

Figure 4.5. With $\lambda=4 \lambda_{\max }$, the rupture time is revealed to be 3765.0 . This is again within $5 \%$ of that published by Oron [2]. The contour plot is qualitatively the same and is depicted here merely for a full comparison with Oron's results. 


\section{FINDINGS}

Various data presentations are used to illustrate the effects of gravity, initial conditions and boundary conditions on interface instability. Film profile plots (2D and 3D) show $h(x, y)$ at a fixed time, usually the time of rupture or time equal to zero. Contour plots illustrate the periodicity in the film profile. The discrete Fourier transform (DFT) plots used here have their direct current (DC) component zeroed out. If the DC component were to be retained, its strength would easily eclipse the strength of the secondary structures on these DFT plots. A film rupture profile and DFT plot are shown in Figure 5.1. In Figure 5.1, the film rupture is depicted on the left and the corresponding wavelength at rupture is captured by the DFT plot on the right. The scale accompanying the DFT plot tracks the magnitude of Fourier energy concentrated in a specific wavenumber (frequency).
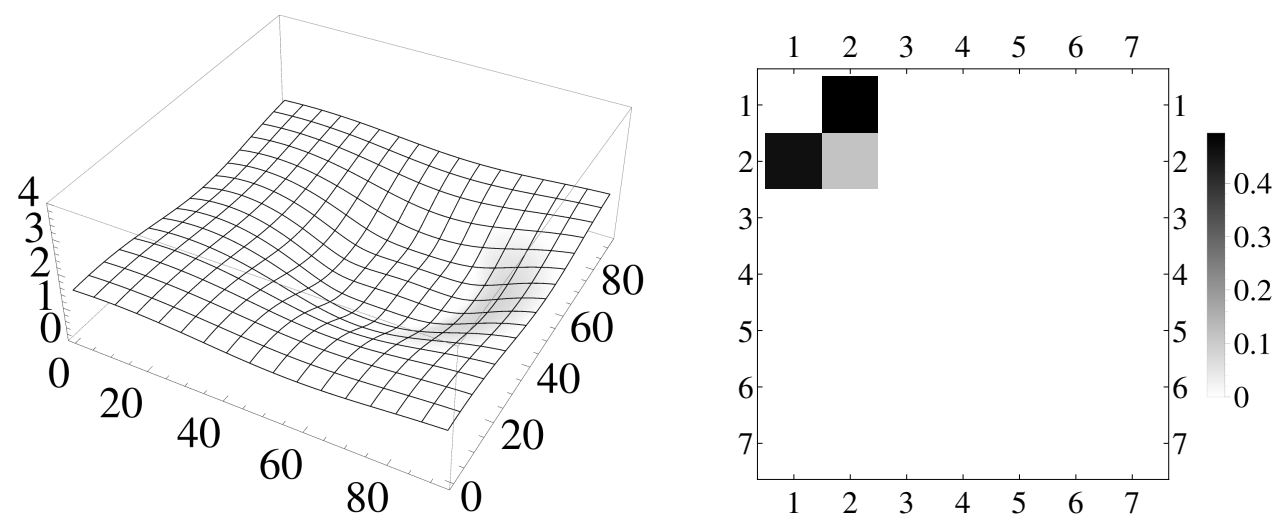

Figure 5.1. Example of how a DFT plot represents wavelength dynamics

\subsection{Mathematical Fluid in Terrestrial \& Zero Gravity}

Understanding the instabilities that affect fluids and liquid films in microgravity is important as capillary forces could become significant in microgravity and could govern the behavior of the liquid system. For instance propulsion systems or water 

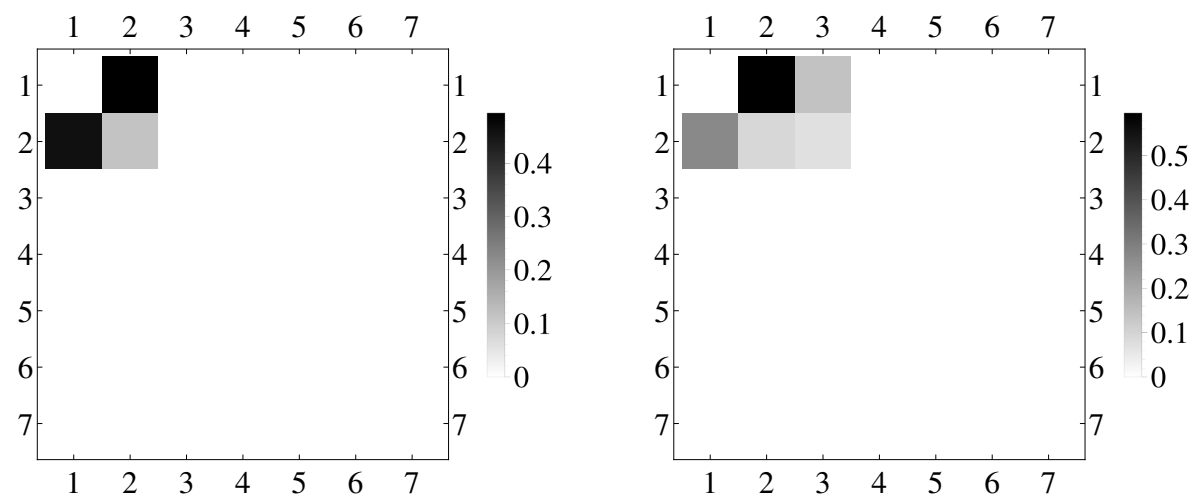

Figure 5.2. DFT plot on the left corresponds to a non-evaporating liquid film in terrestrial gravity. Roughly $40 \%$ of the energy is situated in the fastest growing wavelength. In zero gravity (DFT plot on right), more energy (about 50\%) is stored in the fastest growing wavelength due to the absence of gravity stabilization. The zero gravity case also shows a cascade of energies.

recovery systems that employ or recover liquids would behave differently in low gravity as compared to a terrestrial gravity situation. Evaporation in such systems, for instance, may not allow the liquid and the gas phases to separate into two distinct components because of a lack of buoyancy [32].

Material science provided a major thrust for research in microgravity as well. Gravity provides convective effects which have an effect on the morphology of crystal structures as crystals are generally grown from a liquid state. Growing crystals in a reduced gravity environment omits any convective buoyancy effects that the process would encounter in terrestrial environments. The other effect of microgravity is the ability to process materials without the need for a container.

As with the mathematical fluid with $G=0.333$, similar trends are seen in zero gravity with $G=0.0$. The difference between these cases is captured in the DFT plots shown in Figure 5.2. At rupture in zero gravity, more of the energy is captured in the most significant frequency with the energy spreading to higher harmonics with an increase in domain size. This shows that the secondary structures grow to a larger amplitude in zero gravity than with $G=0.333$. Rupture due to long wave instabilities occurs at an earlier time than in the case when film dynamics are damped by gravity.

\subsubsection{Maximizing Wavelength}

It is observed that in a zero gravity environment, as Bond number $B o=0$, the fastest growing wavelength is greater (maximizing wavenumber is smaller) as compared to cases in terrestrial gravity (non-zero $B o$ ), for the same film thickness. The fastest 
growing wavenumber is plotted in Figure 5.3 for slow evaporation, and in Figure5.5 for fast evaporation. The ratio of the fastest growing wavelengths for non-zero $B o$ and zero $B o$ cases is plotted in Figure 5.4 for slow evaporation and , Figure 5.6 for fast evaporation.

A comparison of film dynamics in zero gravity is also made in Figure 5.7. It is observed that time to rupture is quicker in zero gravity since the growth of thermocapillary structures proceeds without the interference of gravity. This comparison shows that for evaporating liquid films, whether in zero gravity or with non-zero $B o$, the fastest growing wavenumber (and subsequently the wavelength) are not a constant value but change with time.

It must be noted that one of the assumptions made for the development of the evolution equation is that viscous time scales are shorter than evaporative time scales (slow evaporation). The Figures 5.5, 5.6 need to be scrutinized further since the evaporation number of $E=0.1$ could have resulted in evaporation rates so swift that the viscosity driven long wave structures were not given time to manifest.

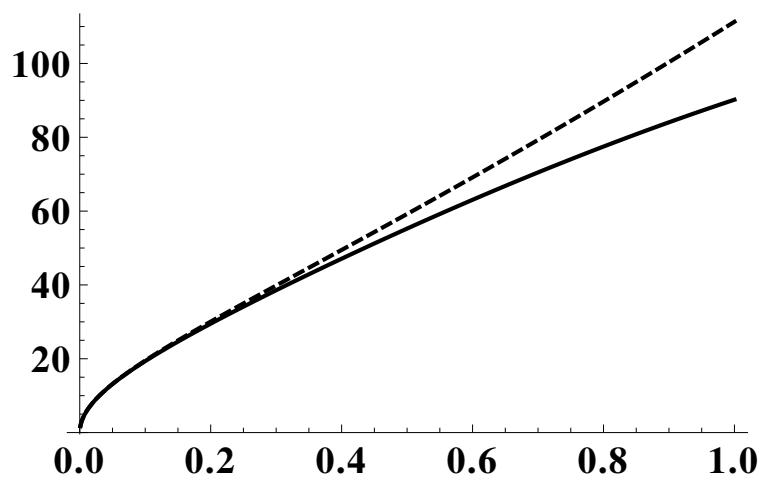

Figure 5.3. Non-dimensional maximizing wavelength plotted as a function of non dimensional film thickness, $h$, for an evaporating liquid film for "slow evaporation", $E=0.0001$. Dashed line is zero gravity, $G=0$ and solid line is when $G=1 / 3$. 


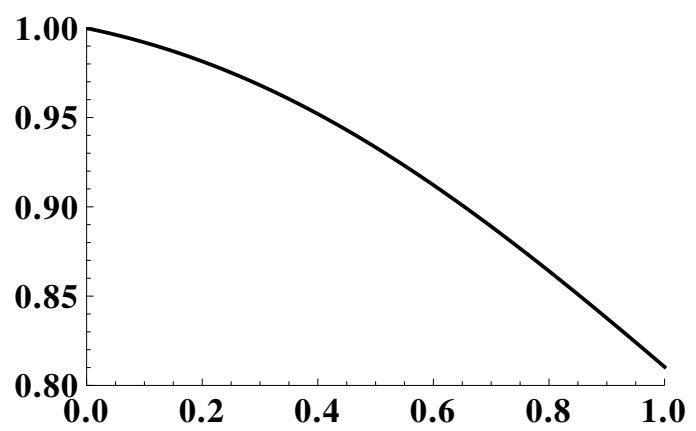

Figure 5.4. Ratio of non-dimensional maximizing wavelengths $\lambda_{\max } / \lambda_{\max \text {,zero g plot- }}$ ted as a function of non dimensional film thickness, $h$, for an evaporating liquid film for "slow evaporation", $E=0.0001$.

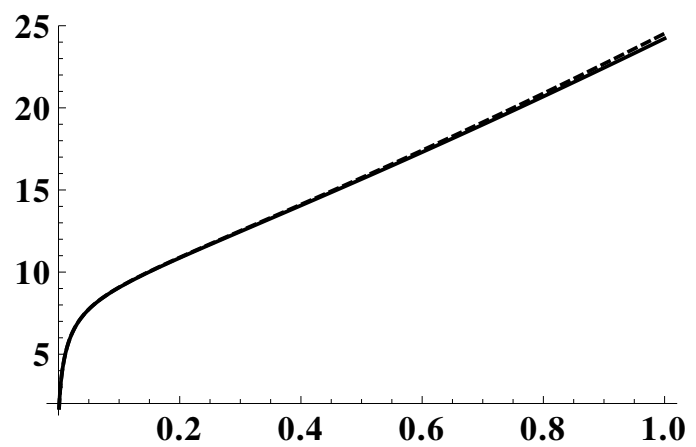

Figure 5.5. Non-dimensional maximizing wavelength plotted as a function of non dimensional film thickness, $h$, for an evaporating liquid film for "fast evaporation", $E=0.1$. Dashed line is zero gravity, $G=0$ and solid line is when $G=1 / 3$.

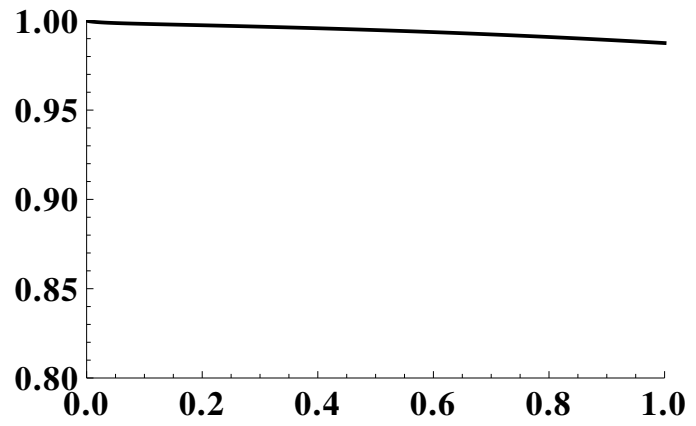

Figure 5.6. Ratio of non-dimensional maximizing wavelengths $\lambda_{\max } / \lambda_{\max \text {,zero g plot- }}$ ted as a function of non dimensional film thickness, $h$, for an evaporating liquid film for "fast evaporation", $E=0.1$. 

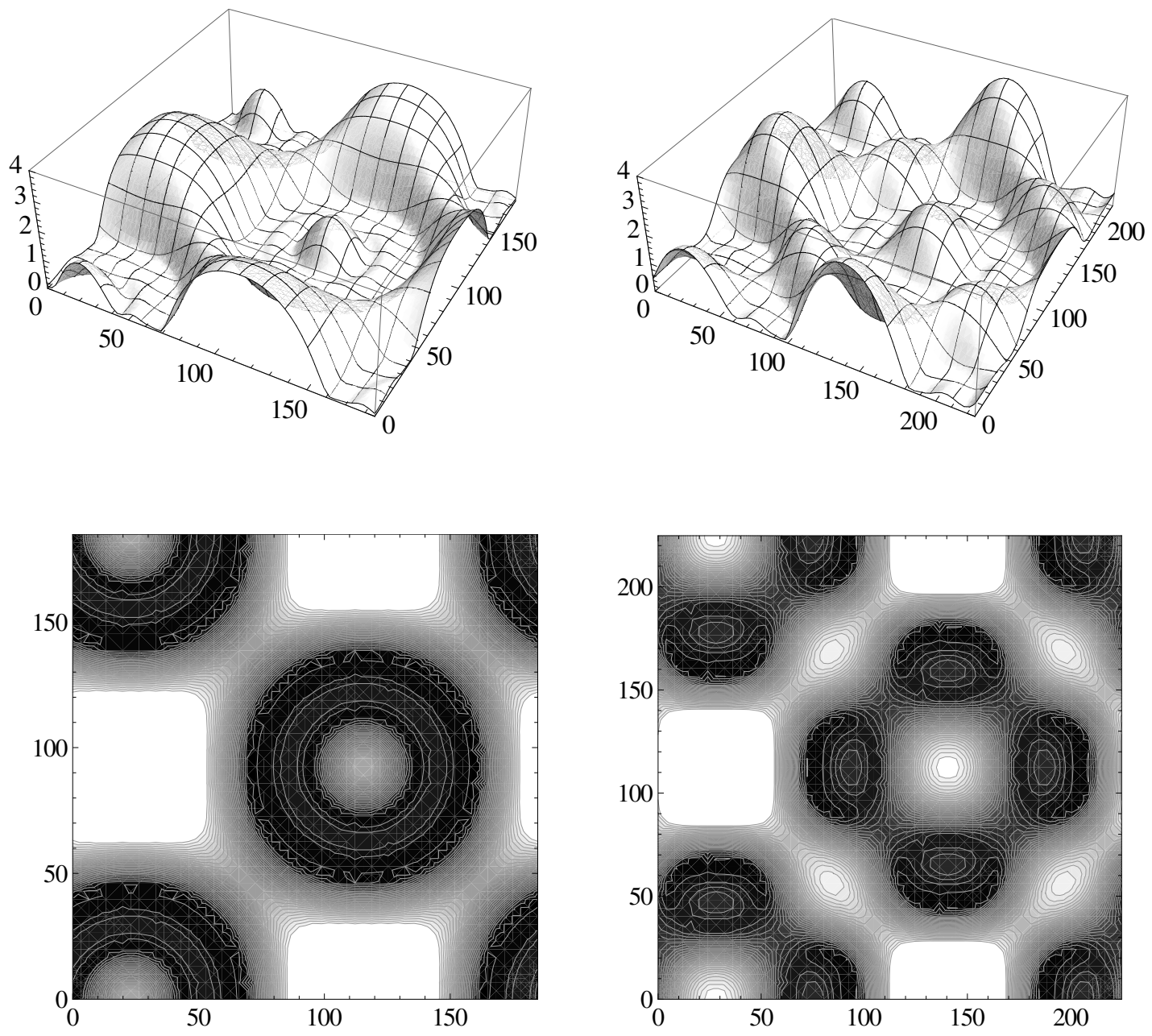

Figure 5.7. Left is Oron's case [2]; right is the same case with $G=0.0$. Rupture times are 2032.0 and 1700.8 , respectively.

\subsubsection{Non-Evaporating Mathematical Fluid - Terrestrial Gravity}

The mathematical fluid properties [1, 2] are used to model the film dynamics. Periodic boundary conditions are utilized laterally and the effect of the "domain size" and the perturbation wavelength (wavenumber) is examined. Three different domain sizes are used with a varying perturbation wavenumber. The domain sizes are $L=\lambda_{\max }, 2 \lambda_{\max }$ and $3 \lambda_{\max }$. The perturbation wavenumbers are $\mathrm{q}=\mathrm{q}_{\max }, \mathrm{q}=2 \mathrm{q}_{\max }, \mathrm{q}=3 \mathrm{q}_{\max }$. Note that when a domain size of $L=\lambda_{\max }$ is used with a perturbation of $\mathrm{q}=\mathrm{q}_{\max }$, the film is subject to the fastest growing wavelength. Any other combination of domain size and perturbation wavenumber results in destabilizing wavelengths other than the fastest growing one. 

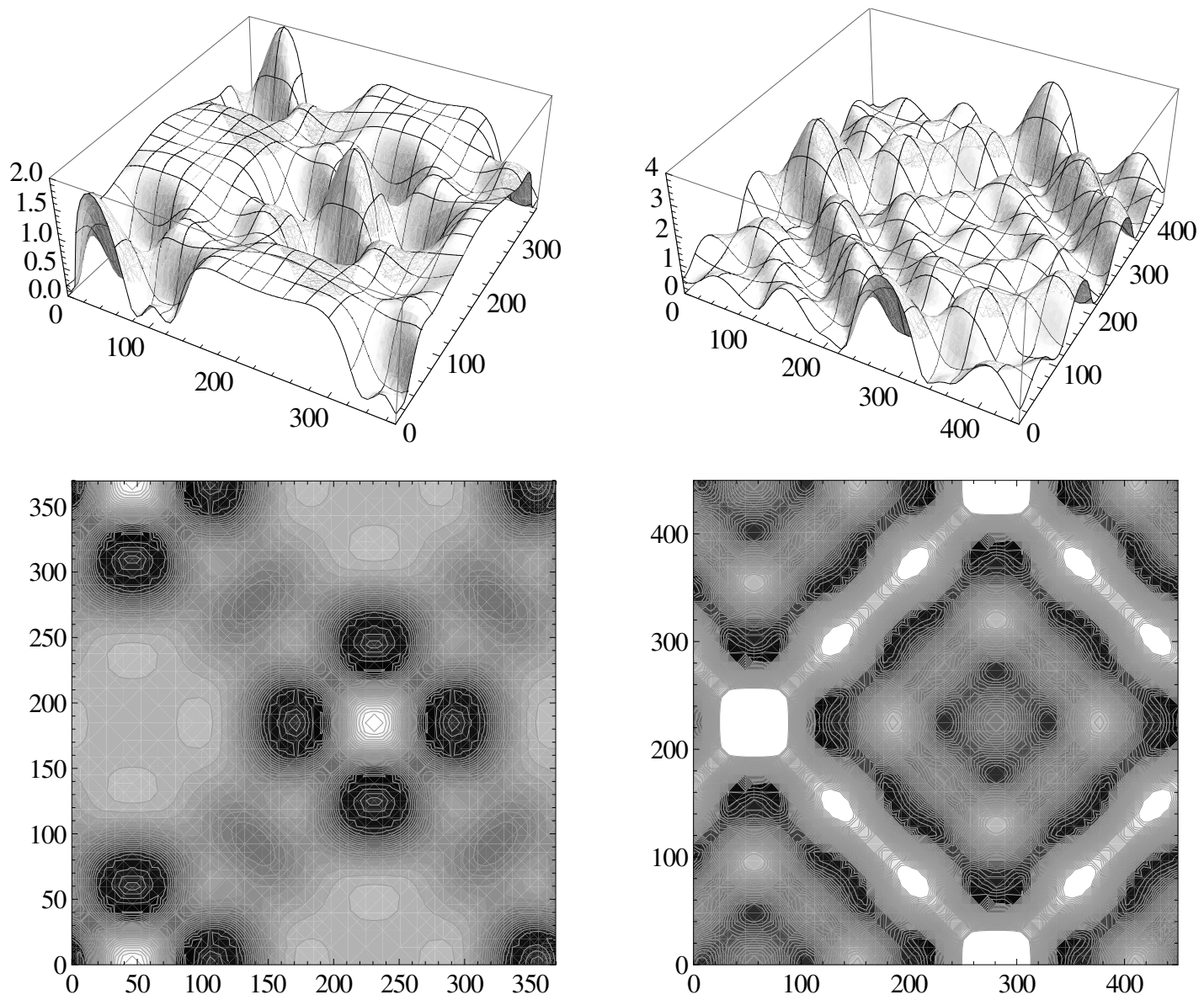

Figure 5.8. Left is Oron's case [2]; right is the same case with $G=0.0$. Rupture times are 3765.0 and 3435.8 , respectively.

It is observed that as the domain size is increased, the complexity of emerging structures increases - secondary and tertiary structures form. This phenomena is captured in discrete Fourier transform plots shown in Figure 5.9. At rupture about $50 \%$ of the entire energy is consistently captured in the fastest growing mode. As domain size is increased for the same perturbation wavenumber, this energy is spread into higher harmonics through a close cascade of energies in some cases.

\subsubsection{Slowly Evaporating Mathematical Fluid - Terrestrial Gravity}

The evaporation number is artificially set to 0.0001 . This describes a "low" propensity for evaporation. This evaporation number is chosen so that its magnitude is similar to that for real fluids vulnerable to long wave instabilities.

Similar to non-evaporating liquid films with $G=0.333$, at rupture most of the 

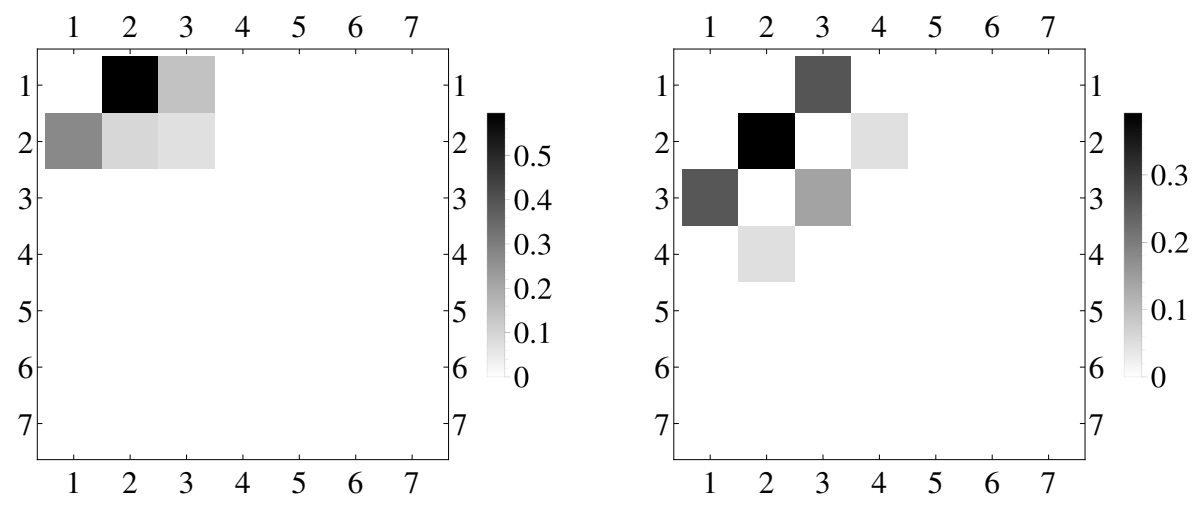

Figure 5.9. As the domain size is increased from $L=\lambda_{\max }$ (left) to $L=2 \lambda_{\max }$ (right), higher harmonics appear.

energy is concentrated in the fastest growing wavelength/frequency and this energy spreads to higher harmonics as the domain size is increased. The structures are different as now the vapor recoil and evaporation terms are in play as well. It must be noted that the vapor recoil term has little bearing on the fastest growing wavelength but since it is a third order term. The significance is tangible only close to rupture as was noticed through $2 \mathrm{D}$ simulations.

\subsubsection{Slowly Evaporating Mathematical Fluid - Zero Gravity}

The mathematical fluid properties are again used to model the film dynamics. However, in this run, the value of $G$ is set to 0 for zero gravity conditions. Periodic boundary conditions are utilized and the effect of the "domain size" and the perturbing wavelength (wavenumber) are again examined. The evaporation number is again artificially set to 0.0001 (low rate of evaporation). It is observed from Figure 5.10 and Table 5.1, that for liquid films in zero gravity or in a non-zero gravity environment, vapor recoil has an effect on film dynamics.

Table 5.1. Effect of vapor recoil on rupture time and amplitude per Figure 5.10 .

\begin{tabular}{lll}
$G=0.333$ & & $G=0.0$ \\
\cline { 1 - 1 } Novap (solid), $T_{\text {rup }}=2568$ & & No Evap (solid), $T_{\text {rup }}=1987$ \\
Slow evap (dotted), $T_{\text {rup }}=1806$ & & Slow evap (dotted), $T_{\text {rup }}=1236$
\end{tabular}



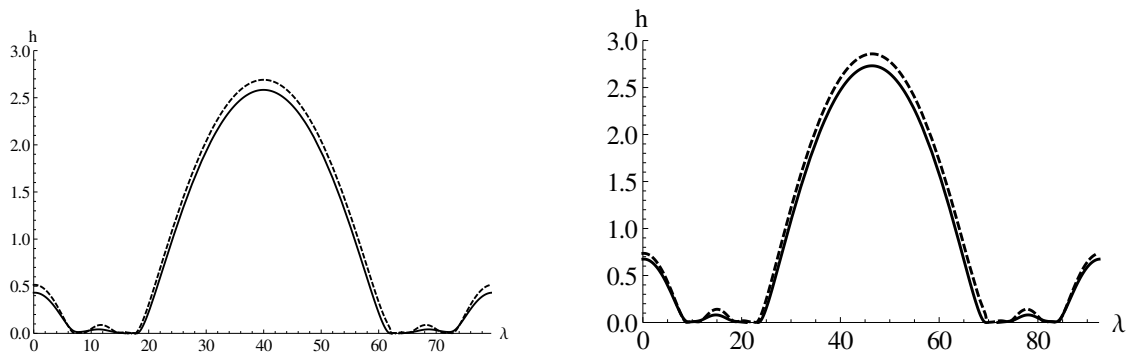

Figure 5.10. The effect of vapor recoil. Figure on left is the mathematical fluid evaporating in non-zero gravity $(\mathrm{G}=0.333)$. Figure on the right is the mathematical fluid evaporating in zero gravity $(G=0.0)$. Zero gravity evaporation allows for thermocapillary structures to grow to marginally higher amplitudes.

\subsubsection{Zero Gravity Summary}

1. As the domain size increases, the complexity of structures increases.

2. When the fastest growing wavelength (frequency) is applied, most of the energy close to rupture is situated in this frequency with a clean cascade of energy to higher harmonics.

3. When wavelengths that are a fraction of the fastest growing one are applied, fractional wavelengths appear at rupture, as seen in a close cascade of energies in the DFT plot.

4. In the absence of gravity (when $G=0.0$ ), the thermocapillary structures hold a greater amount of energy than for the case with $G=0.333$ as seen in the DFT plots.

5. In the case of evaporating liquid films, although vapor recoil and evaporative mass flux are small in magnitude $\left(10^{-7}\right.$ and $10^{-4}$ respectively), they do affect the shape of the secondary structures close to rupture. At rupture, evaporating liquid films in regular or zero gravity have secondary structures of a greater amplitude than non-evaporating liquid films. 


\subsection{Effects of Initial Conditions - Mathematical Fluid}

\subsubsection{Perturbation Functions}

A slowly evaporating liquid film (math fluid) $(E=0.0001)$ in zero gravity is perturbed with the following initial conditions.

1. $1-0.05[\cos (2 \pi x / L)+\sin (2 \pi x / L)] \cos (2 \pi y / L)$

This is commonly used initial condition [1, 2, 25].

2. $1-0.05[\cos (2 \pi x / L)+\sin (2 \pi x / L)] \sin (2 \pi y / L)$

This is analogous to initial condition 3 except that the crests and troughs are out of phase by $90^{\circ}$ giving rise to a final structure that is out of phase by $90^{\circ}$.

3. $1-0.05[\cos (2 \pi(x+y) / \sqrt{2} L)+\sin (2 \pi(x+y) / \sqrt{2} L)] \cos (2 \pi(x-y) / \sqrt{2} L)$

This is condition rotated through an angle of $45^{\circ}$.

4. $1-0.05[\cos (2 \pi x / L)+\sin (2 \pi x / L)] \cos (2 \pi y / L) \sin (2 \pi y / L)$

This initial condition shows an X-direction preferred curvature.

5. $1-0.05[\cos (2 \pi x / L)+\sin (2 \pi x / L)](\cos (2 \pi x / L))^{2}$

This initial condition again shows an X-direction preferred curvature.

6. $1-0.05 \cos (2 \pi y / L)$

This initial condition is purely a function of $y$. It is used to compare and assess the efficacy of $2 \mathrm{D}$ simulations vs the more computationally demanding 3D simulations.

A change in domain size or the percentage amplitude of a perturbation creates a change in curvature. For different situations of changing curvature, fluid flow is driven differently giving rise to a variegated array of final structures as seen in the simulations.

\subsubsection{Changing Amplitude with a Fixed Domain Size}

A cascade of structures and the appearance of superharmonics is observed in all film evaporation cases (zero gravity or otherwise) when the domain size is increased. It is proposed that a change in curvature induces this cascade. The amplitude is held constant at $5 \%$ of the initial film thickness irrespective of the domain size.

To test this theory, the initial condition is chosen as

$$
1-0.05(\cos 2 \pi x / L+\sin 2 \pi x / L) \cos (2 \pi y / L)^{2} .
$$

The amplitude of perturbation is changed from $5 \%$ to $15 \%, 35 \%$ and $50 \%$ of the initial film thickness $\left(h_{0}\right)$. The domain size is held constant at $L=\lambda_{\max }$. 
Figure 5.11 reveal that as the amplitude is increased from $0.15 h_{0}$ to $0.50 h_{0}$ the film dynamics are affected with the film being driven to rupture earlier for larger amplitude perturbations.

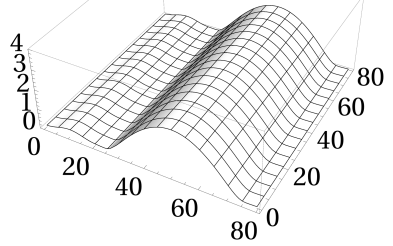

(a) $15 \%$

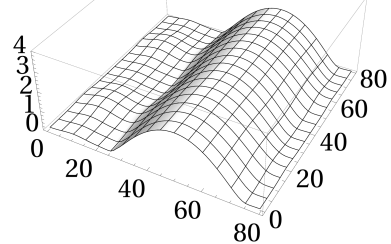

(b) $35 \%$

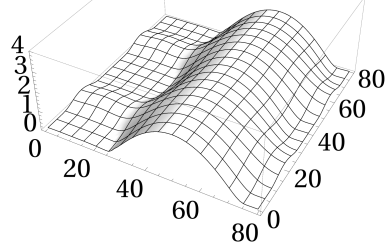

(c) $50 \%$

Figure 5.11. Film profile for an evaporating "mathematical" liquid film in zero gravity. $E=0.0001, G=0.0, S=100, M=35.1, \operatorname{Pr}=7.02$. The amplitude of perturbation is changed from $15 \%$ of the initial film thickness $h_{0}\left(1.15 h_{0}\right)$ to $1.50 h_{0}$. Domain size, $L=\lambda_{\max }$. "Cosine squared" initial condition is used.

The magnitude of the surface tension term, viz., $\left(h^{3} h_{\mathrm{xxx}}\right)_{\mathrm{x}}$ and the thermocapillarity term, $\left(\left(\frac{h^{2}}{h+K}\right)_{\mathrm{x}}\right)_{\mathrm{x}}$ at $T=0$ and $T=T_{\text {rupture }}$ are recorded for this amplitude change in Tables 5.2 and 5.3 .

Table 5.2. Surface tension term

\begin{tabular}{llll} 
Amplitude $\%$ of $h_{0}$ & $15 \%$ & $35 \%$ & $50 \%$ \\
\hline Initial Condition & $6.01 \times 10^{-5}$ & $2.29 \times 10^{-4}$ & $1.901 \times 10^{-4}$ \\
Rupture Condition & $9.33 \times 10^{-4}$ & $9.93 \times 10^{-4}$ & $9.9 \times 10^{-4}$
\end{tabular}

Table 5.3. Thermocapillarity term

\begin{tabular}{llll} 
Amplitude $\%$ of $h_{0}$ & $15 \%$ & $35 \%$ & $50 \%$ \\
\hline Initial Condition & $6.03 \times 10^{-5}$ & $1.71 \times 10^{-4}$ & $2.3 \times 10^{-4}$ \\
Rupture Condition & $3.23 \times 10^{-4}$ & $3.46 \times 10^{-4}$ & $3.53 \times 10^{-4}$
\end{tabular}

From Tables 5.2 and 5.3 , it is observed that for an initial condition, if the domain size is held constant and the amplitude of the perturbation wave increases, the surface tension term increases in magnitude. This is intuitive as surface tension resists increasing curvature. It is also observed that for an initial condition, as the amplitude of the wave increases, it pushes a portion of the liquid further away from the plate 
inducing a larger temperature difference. This has an effect of increasing the magnitude of the thermocapillary term. Thermocapillarity gets stronger with amplitude and the film shows a stronger proclivity to bear secondary structures.

\subsubsection{Changing Domain Size with a Fixed Amplitude}

The amplitude of the perturbation is now held constant at $5 \%$ of the initial film thickness while the domain size is increased from $\lambda_{\max }$ to $3 \lambda_{\max }$. For the "cosine initial condition" and "cosine squared initial condition", the magnitude of the surface tension term and the thermocapillarity term, $\left(h^{3} h_{\mathrm{xxx}}\right)_{\mathrm{x}}$ and $\left(\left(\frac{h^{2}}{h+K}\right)_{\mathrm{x}}\right)_{\mathrm{x}}$ respectively, for $T=0$ are recorded in Table 5.4 .

It is useful to compare the strengths of the surface tension and thermocapillarity terms for the cosine and the cosine squared initial condition for when the ratio of domain size to the perturbation wavenumber is $1\left(L / \lambda_{\max }=1\right)$ in Table 5.4. A single wave is used to perturb the film $(\mathrm{q}=1)$. Hence, the film is perturbed with the maximizing growth rate when $L=\lambda_{\max }$ and a fractional but positive destabilizing growth rate for $L=2 \lambda_{\max }, 3 \lambda_{\max }$.

In Table 5.4. "ST" is the "surface tension term" $\left(\left(h^{3} h_{\mathrm{xxx}}\right)_{\mathrm{x}}\right)$ while "MT" is the "thermocapillarity term" $\left(\left(\left(\frac{h^{2}}{h+K}\right)_{\mathrm{x}}\right)_{\mathrm{x}}\right)$. From comparing the surface tension and thermocapillarity terms within the same growth rate, it is noticed that the surface tension term and the thermocapillarity terms are both greater with a cosine-squared initial condition as compared to a cosine initial condition.

Table 5.4. Comparison of surface tension(ST) $\nabla \cdot\left(h^{3} \nabla \nabla^{2} h\right)$ and thermocapillarity (MT) $\nabla \cdot\left[\left(\frac{h^{2}}{(h+K)^{2}}\right) \nabla h\right]$ terms for same growth rate.

\begin{tabular}{llll}
\hline & $\lambda_{\max }$ & $2 \lambda_{\max }$ & $3 \lambda_{\max }$ \\
\hline ST, cosine & $6.0 \times 10^{-6}$ & $4.0 \times 10^{-7}$ & $8.0 \times 10^{-8}$ \\
MT, cosine & $1.0 \times 10^{-5}$ & $3.0 \times 10^{-6}$ & $2.0 \times 10^{-6}$ \\
ST, cosine-sq & $1.0 \times 10^{-5}$ & $8.0 \times 10^{-7}$ & $1.5 \times 10^{-7}$ \\
MT, cosine-sq & $1.5 \times 10^{-5}$ & $4.0 \times 10^{-6}$ & $2.0 \times 10^{-6}$ \\
\hline
\end{tabular}

The film rupture profiles for these initial conditions are depicted in Figures 5.12 for the cosine initial condition and in Figures 5.13 for the cosine squared initial condition.

It is observed in Figure 5.12 that structures with the cosine initial condition always show the emergence of the fastest growing wavelength with a cascade into superharmonics, as captured by the discrete Fourier transform (DFT) plots at rupture.

With the cosine squared initial condition, as seen in Figure 5.13, there appears 

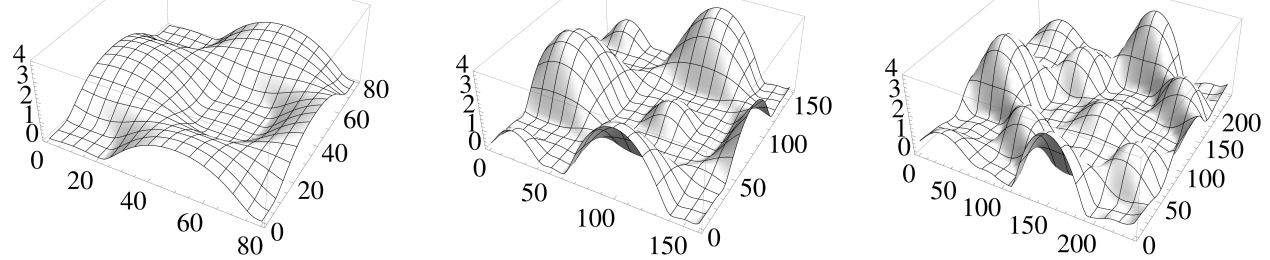

Figure 5.12. Film profile for an evaporating mathematical liquid film in zero gravity. $E=0.0001, G=0.0, S=100, M=35.1, \operatorname{Pr}=7.02$. The amplitude of perturbation is $5 \%$ of initial film thickness. Domain size, $L=\lambda_{\max }$, $2 \lambda_{\max }, 3 \lambda_{\max }$. Cosine initial condition is used.
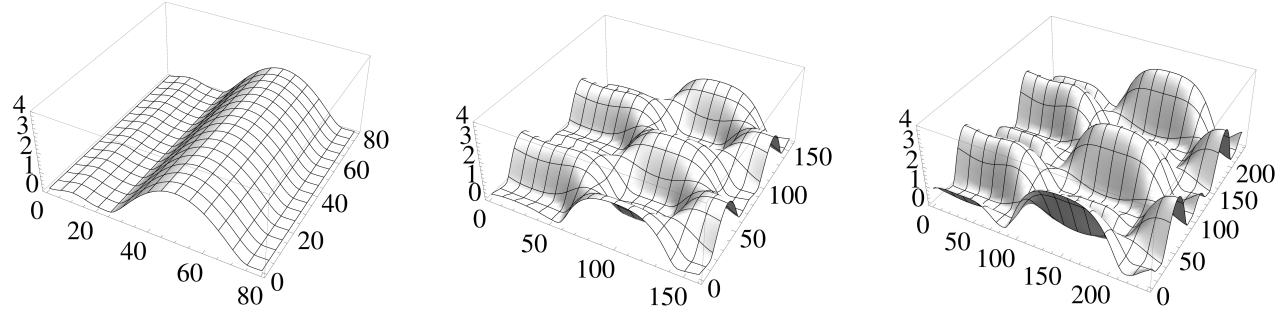

Figure 5.13. Film profile for an evaporating mathematical liquid film in zero gravity. $E=0.0001, G=0.0, S=100, M=35.1, \operatorname{Pr}=7.02$. The amplitude of perturbation is $5 \%$ of initial film thickness. Domain size, $L=\lambda_{\max }$, $2 \lambda_{\max }, 3 \lambda_{\max }$. Cosine squared initial condition is used.

to be a directional preference for the fastest growing wavelength. The cascade of superharmonics captures this directional preference in the respective DFT plots at rupture can be seen in Figure 5.14 .

\subsubsection{Initial Condition Summary}

Increasing the amplitude of perturbation while holding the domain size constant:

1. In Tables 5.2 and 5.3 it is observed that as the amplitude of perturbation is increased while holding the domain size constant, the surface tension term gets stronger for the initial condition. This is logical because as the amplitude increases, the surface gets more curved and larger surface tension strengths are necessary to stabilize the surface.

2. A similar logic follows to explain the increasing strength of the thermocapillarity term for increasing perturbation amplitude. For highly curved surface that occur with increasing amplitude, there exists a stronger non-equilibrium of 

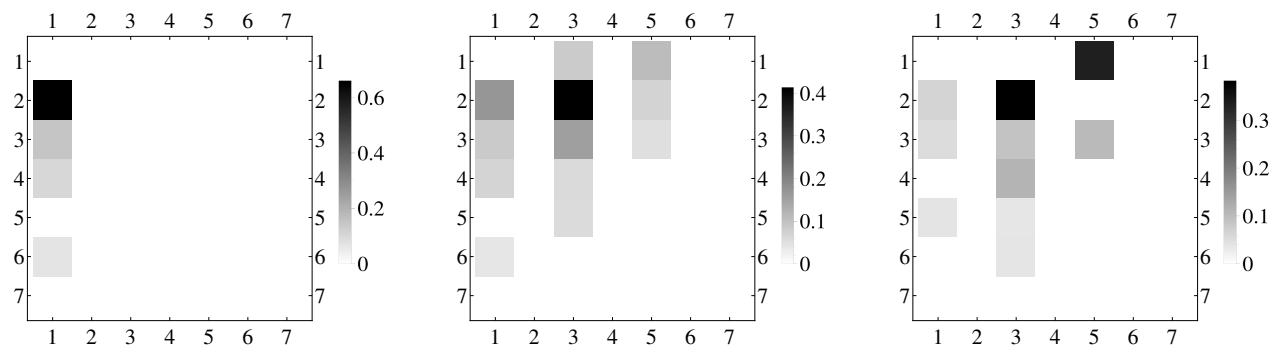

Figure 5.14. Cascade of superharmonics for the cosine-squared initial condition as seen in the DFT plots at rupture

surface temperature distributions as a result of crests of the undulating surface being further away from the heated plate.

3. Increasing the amplitude while holding the domain size constant has the effect of pushing the troughs closer to the hot substrate and the peaks further away. Higher the amplitude of perturbation, stronger the effect of thermocapillarity in destabilizing the film.

Effect of increasing the domain size while holding the amplitude constant:

1. Larger domain size for an initial condition induces a "cascade effect" in the structures.

2. Changing the domain size and/or initial condition begets a change in curvature which affects the strength of surface tension and thermocapillarity terms, both of which are curvature driven.

3. The surface tension and thermocapillarity terms are stronger in the cosine squared initial condition than the cosine initial condition for the same growth rate.

4. Surface tension is stronger in the cosine squared initial condition due to it's higher curvature.

5. Since thermocapillarity is a second order term and has a stronger effect than the fourth order surface tension term, the film is always destabilized.

6. The final structure is thus heavily influenced by the shape and curvature of the initial condition. 
Table 5.5. Comparison of non-dimensional numbers.

\begin{tabular}{|l|l|l|}
\hline & DCM $\left(g=9.81 / 0.0 \mathrm{~m} / \mathrm{s}^{2}\right)$ & Math fluid (regular/zero g) \\
\hline $\mathrm{G}$ & $4.11 \times 10^{6} / 0$ & $0.333 / 0$ \\
$\mathrm{~S}$ & $143 \times 10^{3}$ & 100 \\
$\mathrm{M}$ & $122 \times 10^{3}$ & 35.1 \\
$\operatorname{Pr}$ & 3.9 & 7.02 \\
$\epsilon$ & $6.01 \times 10^{-8}$ & 0 \\
$\delta$ & $5.19 \times 10^{-7}$ & 0 \\
\hline
\end{tabular}

\subsection{Dichloromethane (DCM) in Terrestrial \& Zero Gravity}

The evolution equation is solved for a $2.35 \mathrm{~mm}$ thick Dichloromethane (DCM) liquid film. DCM was selected for its relatively high vapor pressures, good wetting abilities, relative ease of handling in experimental situations, and because it is readily available. The thickness of $2.35 \mathrm{~mm}$ is selected based on memory/RAM limitations. Any thicker and it would be difficult to have confidence on the grid independence of numerical results. The non dimensional parameters for the Dichloromethane liquid film are compared with that of the mathematical fluid in Table 5.5 .

Comparison of film dynamics of an evaporating $2.35 \mathrm{~mm}$ Dichloromethane liquid film from Figures 5.15 and 5.16 reveal interesting characteristics. In earth gravity (Figure 5.16), the Bond number term in the evolution equation is considerably stronger than the strength of thermocapillarity, $m$ and all long wave modes are stabilized by surface tension and gravity and the only significant observation is the finite time evaporation of the film. The growth rate of instabilities calculated from the equation for growth rate shows, concurrent to the observation, a negative growth rate or a damping effect.

However, when the effect of gravity is turned off by setting $g=0.0 \mathrm{~m} / \mathrm{s}^{2}$, shown in Figure 5.15, and hence nullifying the stabilizing effect of the Bond number term, thermocapillarity causes vulnerability to long wave destabilization. The film eventually ruptures in under a second (physical time). Thermocapillary fingers are seen at rupture.

\subsubsection{DCM Summary}

The $2.35 \mathrm{~mm}$ Dichloromethane liquid film evaporates swiftly and with the presence of long wave instabilities in zero gravity while in terrestrial gravity, the long wave modes are damped out by a combined effect of gravity and surface tension. 

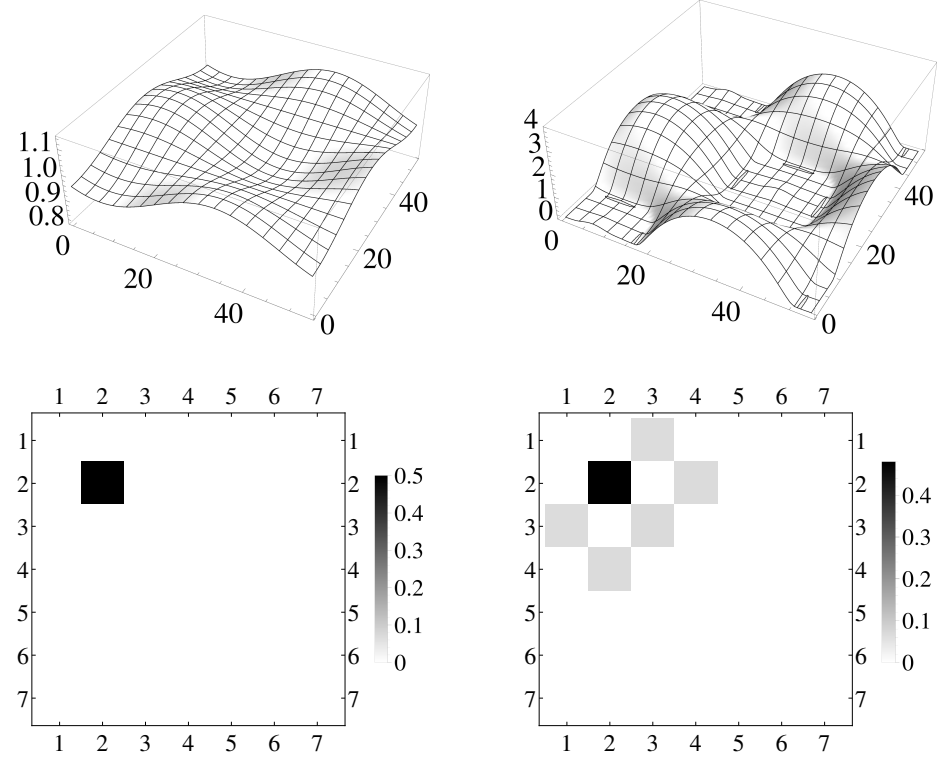

Figure 5.15. Zero gravity evaporation of $2.35 \mathrm{~mm}$ thick DCM. Figure on the left is the cosine initial condition used to perturb the DCM film. Figure on the right shows long-wave destabilized liquid film.
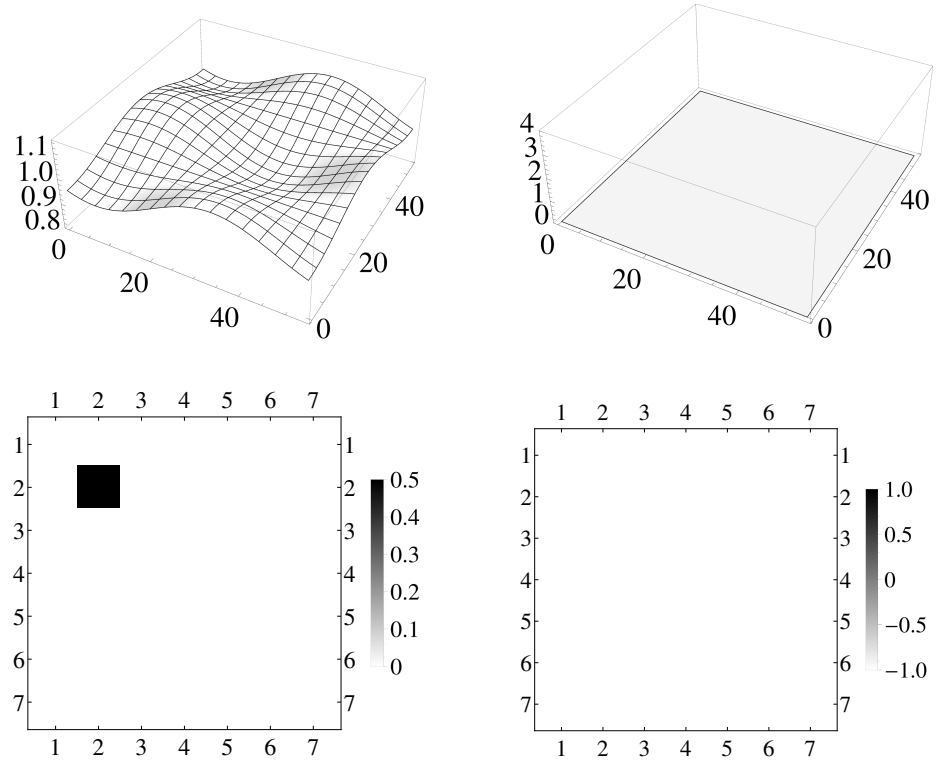

Figure 5.16. Terrestrial gravity evaporation of $2.35 \mathrm{~mm}$ thick DCM. Figure on the left is the cosine initial condition used to perturb the DCM film. Figure on the right shows lack of long-wave instabilities. 
This has technological implications in that long wave instabilities and concurrent rupture occur for a $2.35 \mathrm{~mm}$ thick DCM liquid film in zero gravity. In Earth's gravity the $2.35 \mathrm{~mm}$ DCM film is thick enough to resist long wave destabilization due to gravity stabilization of surface disturbances.

\subsection{Noisy Uniform Initial Conditions - DCM in Zero Gravity}

The domain size in all previous simulation runs was equal to a whole number multiple of the fastest growing wavelength. The domain length was changed to a non-whole number multiple of the fastest growing wavelength with a random initial condition as in Figure 5.17. When the domain length, $L=2.317 \lambda_{\max }$, the DCM film ruptured due to long wave destabilization in zero gravity. The fastest growing wavelength was the strongest harmonic at rupture as seen in the DFT plots.
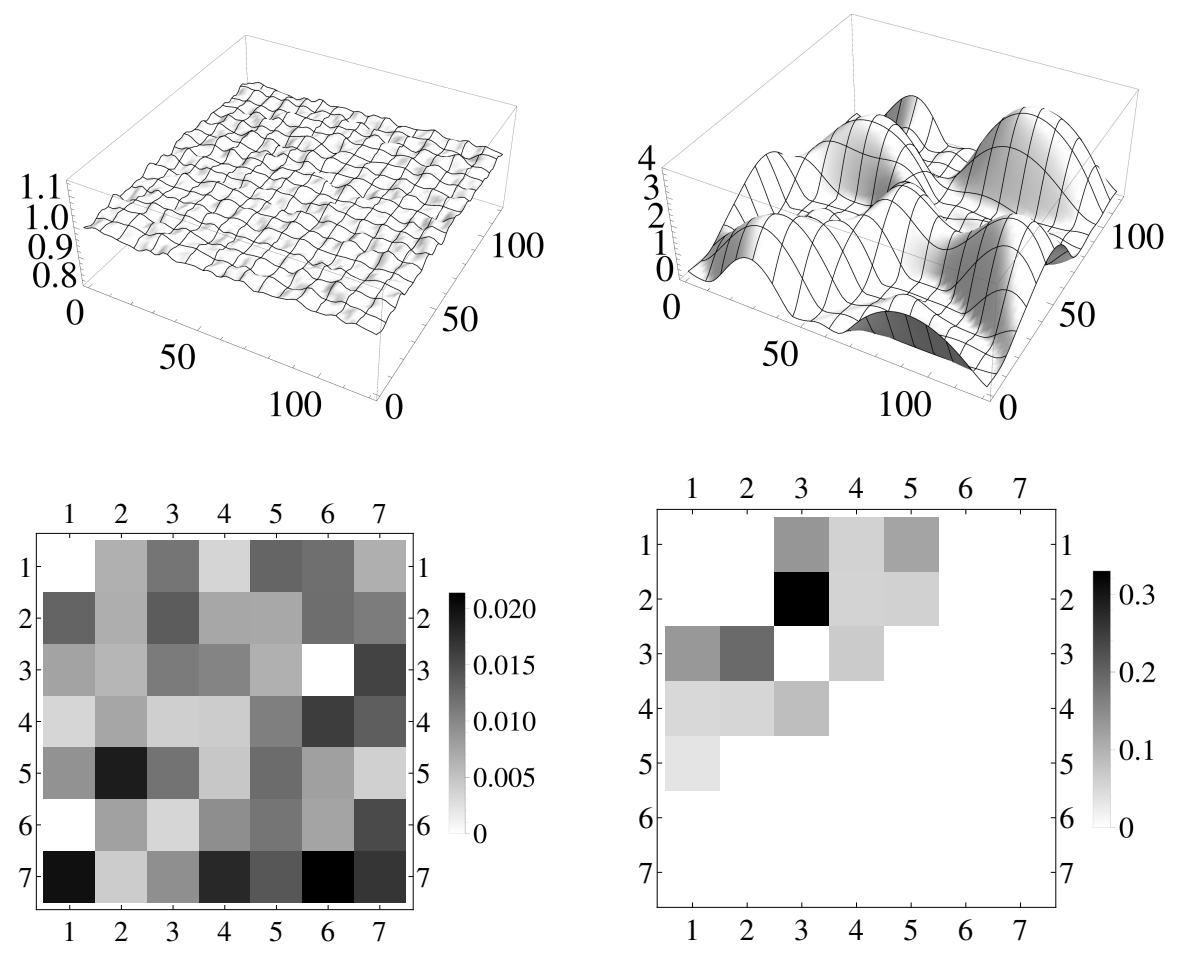

Figure 5.17. Zero gravity evaporation of $2.35 \mathrm{~mm}$ thick DCM with a uniform random perturbation in a domain whose side is a non-whole number multiple of fastest growing wavelength. Figure on the left is the cosine initial condition used to perturb the DCM film. Figure on the right shows long-wave destabilized liquid film. 
When a rectangular domain is used instead of a square domain, the initial curvature is not the same in both directions. Domain dimensions tested are $L x=$ $2.238 \lambda_{\max }, L y=1.641 \lambda_{\max }$. The length and width were arbitrarily chosen and the film profiles are depicted in Figure 5.18. With a rectangular domain, the DCM film ruptured via long wave instabilities in zero gravity. The fastest growing wavelength appeared as the strongest harmonic at rupture as seen in the DFT plots. The thermocapillary structures showed a directional preference and formed lengthwise.
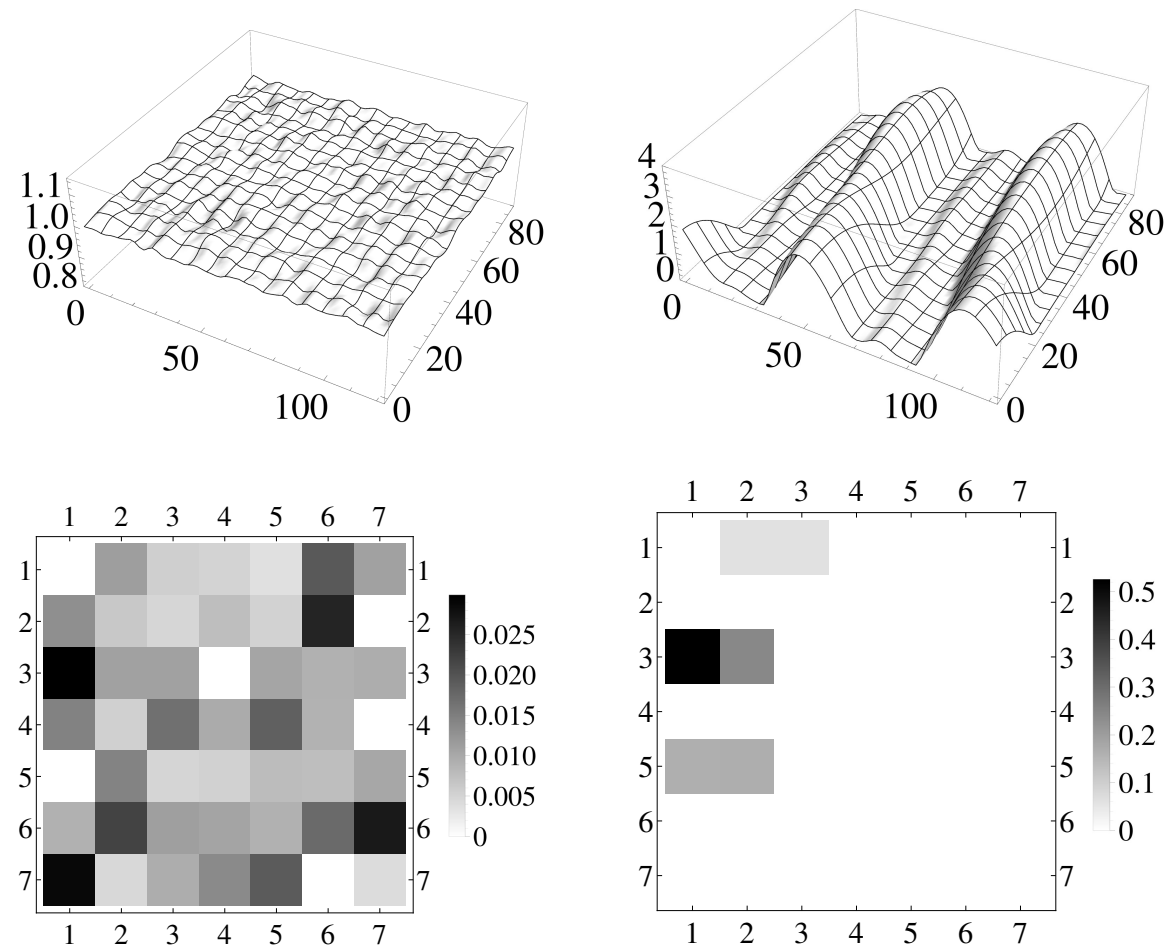

Figure 5.18. Zero gravity evaporation of $2.35 \mathrm{~mm}$ thick DCM with a uniform random perturbation in a domain whose length and breadth are a non-whole number multiple of fastest growing wavelength

\subsection{D vs 3D Simulations}

The efficacy of 2D simulations such as those in 2D CFD simulations 11 and 3D simulations [2] are compared to 3D simulations such as those in Oron [2]. A comparison of film profiles for a slowly evaporating mathematical fluid in zero gravity shows that 2D simulations have swifter run time by several orders of magnitude whilst allowing the user to probe closer to an "adsorbed film" stage for perturbations that are 
not mixed-mode (functions of $\mathrm{X}$ and $\mathrm{Y}$, are termed "mixed-mode" whilst function of only X or only Y are termed "simple mode").

For the 3D case a simple mode initial condition purely in $Y$ is chosen. The film profile (Figures 5.19 and 5.20 ) at rupture reveals a single peak forming from thermocapillary forces.

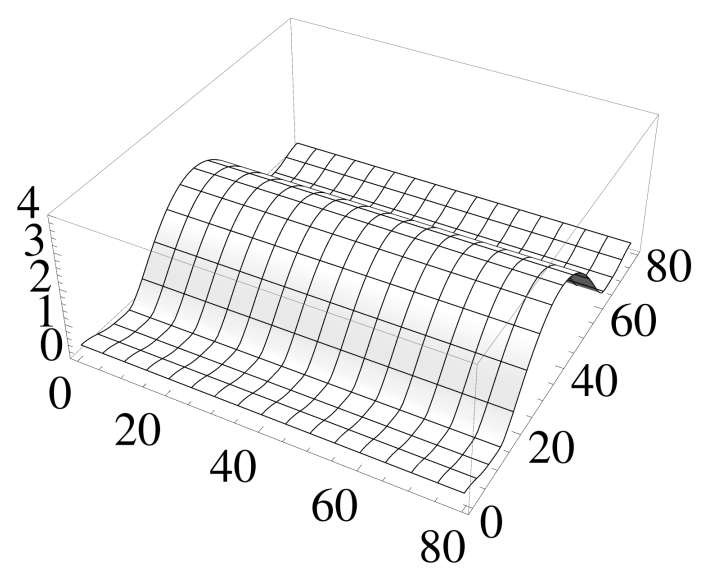

Figure 5.19. Rupture in 3D case

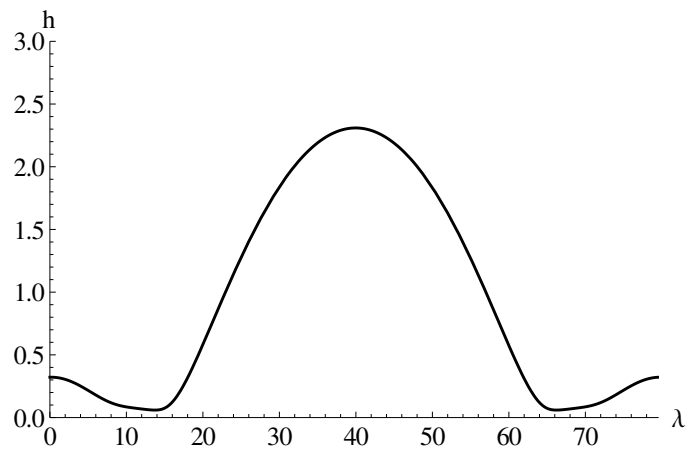

Figure 5.20. Rupture in $2 \mathrm{~d}$ case

The 2D situation is a whole "dimension" lesser than the 3D situation. Hence, the former allows us to use more grid points than what our computational resources allow for the latter case. Hence, increasing the grid points in the 2D simulations to 8 times that of the 3D simulation, it is revealed that at rupture, the film shows the presence of two secondary droplets forming as seen in Figures 5.21 and 5.22 .

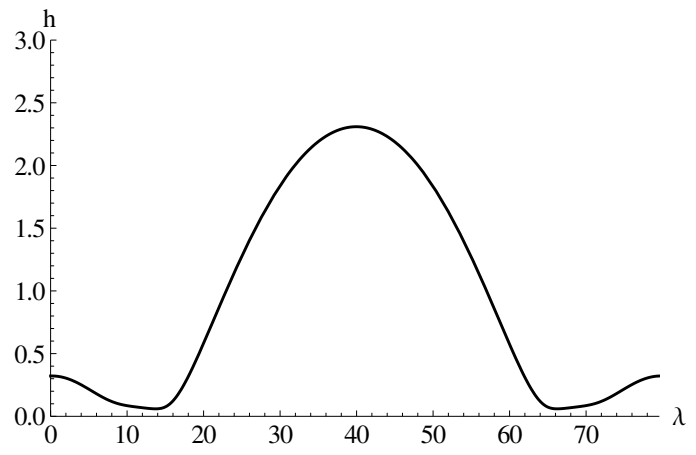

Figure 5.21. Rupture in 3D case at $T=1319.0$

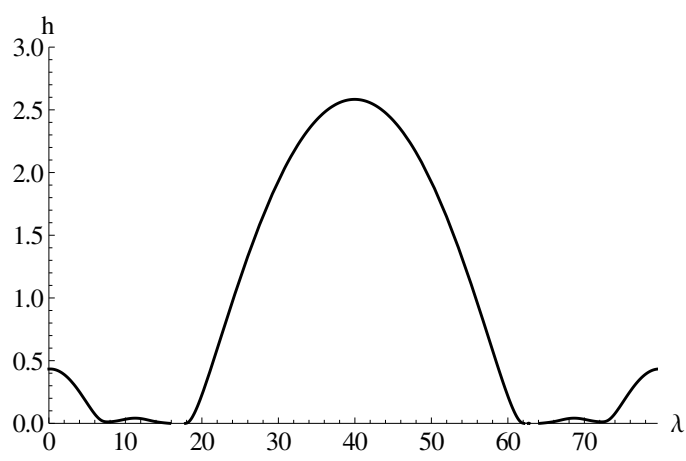

Figure 5.22. default

At this juncture, the definition of rupture time needs to be revisited. It is observed from the comparison of $2 \mathrm{D}$ and $3 \mathrm{D}$ film profile results that the system turns stiff at $T=942.0$ for the $3 \mathrm{D}$ case with a minimum film thickness of roughly $5 \%$ the initial 
mean film thickness. In the $2 \mathrm{D}$ case, the same fluid parameters produce rupture at $T=1250.0$ with stifness being observed by the solver when the film touched the surface while also revealing the presence of secondary droplets unavailable in the 3D case.

We have already bolstered the fact, via 3D simulations, that a growing domain size engendgers more complex structures via secondary drops as compared to smaller domain sizes. However, these larger domain sizes come at a severe computational run time cost for 3D cases (run times in excess of 3 hours).

The effect of a larger domain size with $L=3 \lambda_{\max }$ is used to make a comparison between $2 \mathrm{D}$ and 3D film profiles in Figures 5.23 and 5.24 . There is strong qualitative similarities between 2D and 3D profiles and that stiffness ensued at nearly the same time. The run times were significantly different with the $2 \mathrm{D}$ case clocking less than 10 seconds of run time while the 3D case accrued over 2 hours of run time.

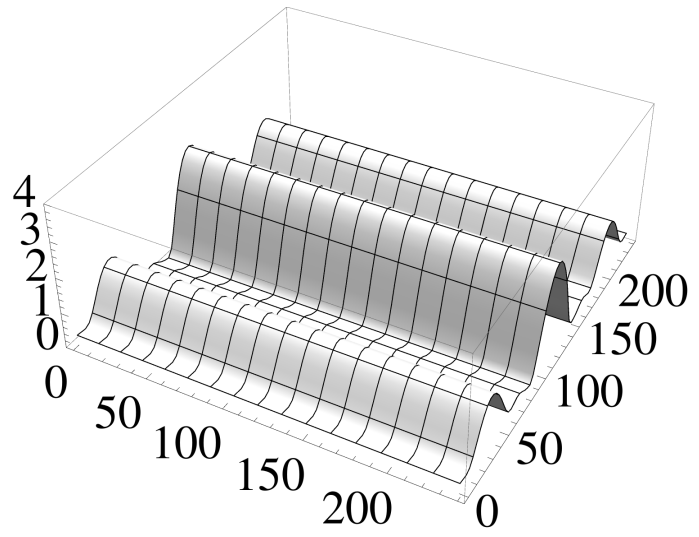

Figure 5.23. Rupture in 3D case at $T=1319.0$

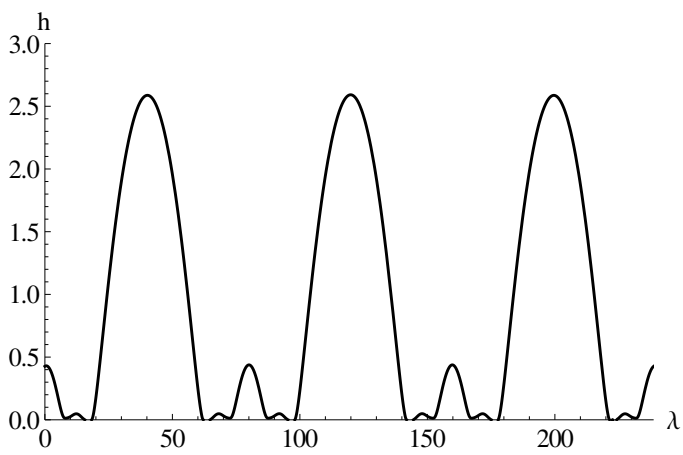

Figure 5.24. Rupture in 2D simulation.

\subsubsection{D vs 3D Simulations Summary}

1. The "rupture time" as used in literature for comparison are strongly subject to computational resources available and are perhaps not the best metric. Hence we have used discrete Fourier transform plots to explain the formation of structures.

2. The benefits of $2 \mathrm{D}$ simulations (superior program run times, larger grid density availability) far outweigh 3D simulations when simple mode dynamics are involved

3. 2D simulations offer a validated numeric tool for simple feasibility analysis 


\subsection{Effects of Boundary Conditions}

\subsubsection{Periodic vs Free and Pinned Boundary Conditions}

Periodic boundary conditions have always been applied to solve the evolution equation since they are the least computationally demanding of the boundary conditions. A comparison is made between periodic and free boundary conditions applied to a slowly evaporating mathematical fluid film for three different domain sizes.

Periodic boundary conditions (for a 1D film case in X and t) are prescribed as in equation 5.1 :

$$
\begin{array}{r}
h(x \text { Min }, t)=h(x \operatorname{Max}, t) \\
\frac{\partial h}{\partial x}(x \operatorname{Min}, t)=\frac{\partial h}{\partial x}(x \operatorname{Max}, t) \\
\frac{\partial^{2} h}{\partial x^{2}}(x \operatorname{Min}, t)=\frac{\partial^{2} h}{\partial x^{2}}(x \operatorname{Max}, t) \\
\frac{\partial^{3} h}{\partial x^{3}}(x \operatorname{Min}, t)=\frac{\partial^{3} h}{\partial x^{3}}(x \operatorname{Max}, t)
\end{array}
$$

As per Becerril et al. [51] and Petsi and Burganos [52], pinned boundary conditions are prescribed as in equation 5.2 and allow for no mass flux through the lateral walls at $x$ Min, $x$ Max via $J(x, t)=0$.

$$
\begin{aligned}
h(x \operatorname{Min}, t) & =1 \\
h(x \operatorname{Max}, t) & =1 \\
J(x \operatorname{Min}, t) & =0 \\
J(x \operatorname{Max}, t) & =0
\end{aligned}
$$

\subsubsection{Free Boundary Condition Summary}

From Figures 5.25, 5.26 and 5.27 , we see that the imposed contact angle at the wall for free boundary conditions is faithfully maintained. However, this engenders stiffness in the equation and the film is affected by thermocapillarity and evaporative mass loss at a much slower rate than for periodic boundary conditions. 


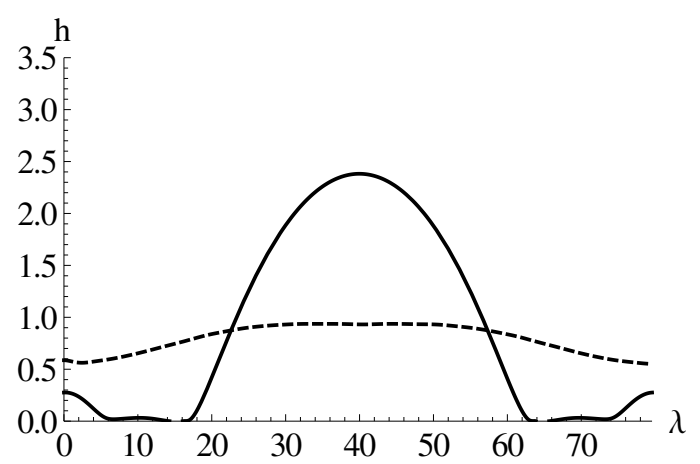

Figure 5.25. Thick line: Slowly evaporating mathematical fluid $(\mathrm{E}=0.0001)$ with periodic boundary condition at rupture. Dashed line: free boundary conditions at the same time free boundary conditions. $L=\lambda_{\max }$

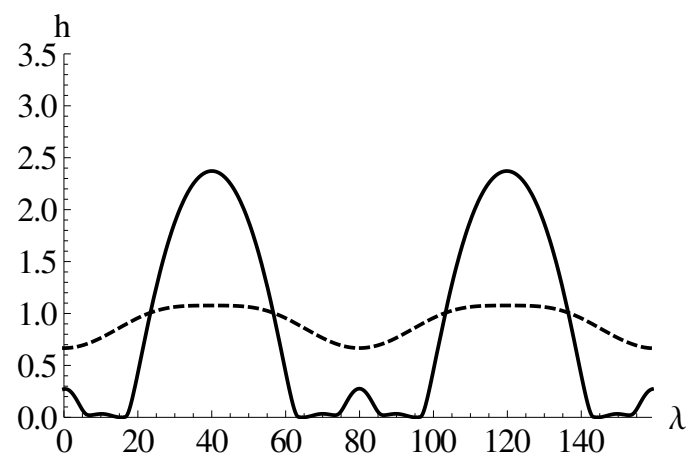

Figure 5.26. Thick line: Slowly evaporating mathematical fluid $(\mathrm{E}=0.0001)$ with periodic boundary condition at rupture. Dashed line: free boundary condition at the same time with free boundary conditions. $L=2 \lambda_{\max }$

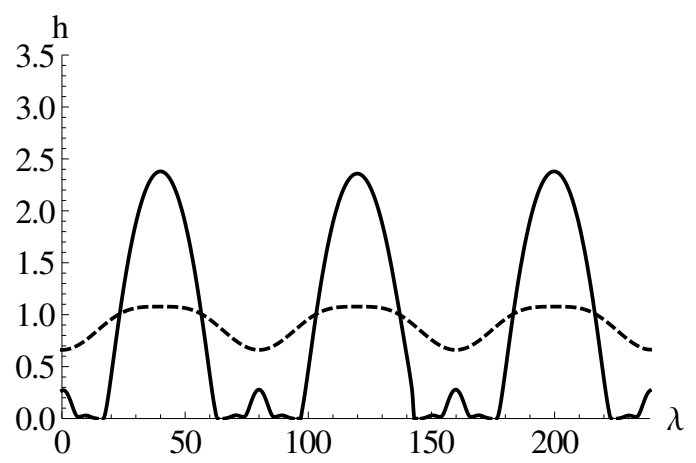

Figure 5.27. Thick line: Slowly evaporating mathematical fluid $(\mathrm{E}=0.0001)$ with periodic boundary condition at rupture. Dashed line: free boundary conditions at the same time with free boundary conditions. $L=3 \lambda_{\max }$ 


\subsection{Comparison of Evolution Equations}

The modified evolution equation (equation 5.3) is solved with an increasing influence of buoyancy driven instabilities as defined by the term $R\left(h^{3} h_{x}\right)_{x}$ where $R=$ $R a / \operatorname{Pr} S$. For a constant domain size and perturbation wavelength, the film profile with and without buoyancy effects is examined in Figure . The choice of Rayleigh number is based on an order of magnitude analysis on real fluids such as DCM and silicone oil with sub-millimeter thicknesses.

$$
\begin{array}{r}
h_{T}+\frac{E}{(h+K)}+S\left(h^{3} h_{X X X}\right)_{X}-\frac{G}{3}\left(h^{3} h_{X}\right)_{X}+E^{2} D^{-1}\left[\frac{h^{3} h_{X}}{(h+K)^{3}}\right]_{X}+ \\
K_{M P^{-1}}\left[\frac{h^{2} h_{X}}{(h+K)^{2}}\right]_{X}+\underbrace{\frac{5 R a}{48 \operatorname{Pr}}\left[\frac{K^{2}}{(h+K)^{2}} h^{4}+h^{4}\right] h_{X}}_{\text {buoyancy driven instabilities }}=0
\end{array}
$$

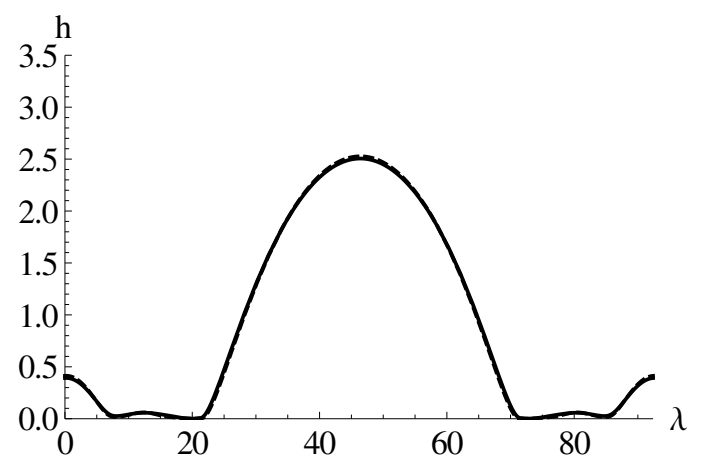

Figure 5.28. Thick line: Slowly evaporating mathematical fluid $(E=0.0001)$ without the buoyancy term. Dashed line: Full evolution equation with $R=10^{-4}$. $L=\lambda_{\max } . T_{\mathrm{Rup}, \text { without } \mathrm{Ra}}=1781, T_{\mathrm{Rup}, \text { with } \mathrm{Ra}}=1607$.

\subsubsection{Evolution Equation Summary}

It is observed that the influence of buoyancy for films of sub-millimeter thickness is negligible. However, we consider buoyancy driven instabilities in the evolution equation since this paves the way for investigating transient long wave effects reported by Kimball [53]. These transient long wave effects reported by Kimball [53] are similar to the thermocapillarity driven instabilities observed in the long wave formulation. 


\section{Contributions to the Field}

\section{Reconstitution of the evolution equation}

The evolution equation as described by Burelbach et al. [25] and Oron [2] has been reworked to include the effect of buoyancy driven convection. This was done to possibly explain transient surface tension driven phenomenon as reported by our collaborators (Kimball [53]). It is noticed that in case of an evaporating liquid film, the constitutive relationship is used to express evaporation dynamics at the interface. In case of non-evaporating liquid films, a heat transfer condition (Robin boundary condition) is used at the interface, to link liquid and vapor sides. Our equation allows for the simulations of either case. It is observed that across literature, the Marangoni parameter has been defined in a variegated manner by sometimes weighting it with a Biot number or a modified Biot number (a function of the non-equilibrium constant, $K)$. Our reformulation of the evolution equation placates this discrepancy.

\section{Three-dimensional zero gravity data for the mathematical fluid and DCM}

The evolution equation was solved with the mathematical fluid parameters for cases of zero acceleration due to gravity. This was done to provide supporting evidence for experiments being conducted at the university of Washington, by our collaborators. So far, zero gravity simulations have not been performed in literature. As per our validated numeric code, the fastest growing wavelength appears in zero gravity although the wavelength is now shorter (both in regular and zero gravity). We also have data for evaporating liquid films that suggests that the fastest growing wavelength changes with film thickness as the film evaporates (both in regular and zero gravity). This data for evaporating liquid films or that for zero gravity cases, to the authors knowledge, has not been reported.

The author also performed simulations with Dicholoromethane as the test fluid. The results obtained were faithful to previous trends registered with the mathematical fluid. 


\section{Effect of initial condition}

Our evolution equation and validated numeric code proves that the choice of initial conditions governs the film dynamics (evaporating or non-evaporating). The choice of initial conditions is shown to change the initial curvature which eventually has an effect on the strength of various forces being modelled by the evolution equation. To circumvent the effect of initial conditions, stochastic white noise is used as an initial condition. With a white noise initial condition, the fastest growing wavelength was recovered (observed as the strongest or brightest frequency in DFT plots) with evaporating and non-evaporating liquid films and zero and regular gravity, with both the mathematical fluid and Dichloromethane. The white noise initial condition was coupled with a rectangular periodic domain instead of the usual square periodic domain. The wavelength trends did not change with a change in the shape of the domain. Domain sizes (length and breadth dimensions) of non-whole number multiples of the fastest growing wavelength were also used to negate the choice of domain size. It was observed that with all these various permutations and combinations of initial conditions, domain shapes, domain sizes, the fastest growing wavelength was always recovered.

\section{Two-dimensional vs three-dimensional simulations}

Two-dimensional simulations were compared with three-dimensional simulations for simple mode initial conditions (initial conditions that are purely functions of $X$ or $Y$ ). It is oberved that, for simple modes of a perturbation, the two-dimensional simulation demands lesser computational memory, hence allowing us to glean film dynamics closer to rupture. Two-dimensional simulations are useful in providing proof of a concept and feasibility analysis because of their swift computing times.

\section{Free and pinned boundary condition}

Free and pinned boundary conditions were used for two-dimensional simulations so as to observe the effect of boundary conditions on overall film dynamics. The equations got stiffer as we moved from periodic to free to pinned boundary conditions. Large domain sizes with pinned boundary conditions took the longest to solve. With current computational capabilities, free and pinned boundary conditions cannot be applied to three-dimensional films without significant memory overhead. This is because with the usual periodic boundary conditions, the three dimensional film

behaves like a membrane. With free or pinned boundary conditions, the film behaves like a plate, and significantly stiffer. 
APPENDICES 


\section{A. Modified Constitutive Relationship}

The constitutive relationship derived by Wayner [54] describes the relation between mass flux at the interface and the causes of the jump in pressures due to curvature effects and disjoining pressure. The effect of gravity is included for vertical films. It is an extension of that derived by Schrage [55] and is given by Equation 1.1 .

$$
J_{\mathrm{W}}=C_{1}\left(\frac{M_{w}}{2 \pi R_{g} T^{I}}\right)^{1 / 2}\left[\frac{P^{v} M_{w} L\left(T^{I}-T^{v}\right)}{R_{g} T^{I} T^{v}}-\frac{V_{l} P^{v}(\Pi+\sigma \kappa)}{R_{g} T^{I}}+\frac{M_{w} g P^{v} x}{R_{g} T^{v}}\right]
$$

All terms with a superscript $v$ refer to vapor quantities, those with superscript $I$ refer to interfacial quantities, those with subscript $l$ refer to liquid quantities. Equation 1.2 was used by Burelbach et al. [25]. to describe their constitutive relationship. This was referred to as an "ideal constitutive relationship". Clearly this does not include curvature and disjoining pressure effects.

$$
J_{\text {id }}=C_{1}\left(\frac{M_{w}}{2 \pi R_{g} T}\right)^{1 / 2}\left(\frac{P^{v} M_{w} L}{R_{g} T^{v} T^{I}}\right)\left(T^{I}-T^{v}\right)
$$

Since we now have two mass flux $(J)$ equations. We have the mass flux ratio as given in equation 1.3 .

$$
\begin{gathered}
J_{\text {id }}=\frac{T^{I}-T^{v}}{K} \\
J_{\mathrm{w}}=\frac{\left(T^{I}-T^{v}\right)-\chi_{\mathrm{w}}}{K}
\end{gathered}
$$

Therefore,

$$
\frac{J_{\mathrm{w}}}{J_{\mathrm{id}}}=1-\frac{\chi_{\mathrm{w}}}{\left(T^{I}-T^{v}\right)}
$$

where

$$
\chi_{\mathrm{w}}=\left(T^{I}-T^{v}\right) \frac{(\sigma \kappa+\Pi)}{M_{w} L} V_{l}
$$

In a future discourse with the evolution equation, we could include $\chi_{w}$ to handle gravity, curvature and disjoining pressure effects on non-equilibrium at the film interface. 


\section{B. Compendium of Results}

The appendix of results that follows acts as a review of all the numerical cases run to draw the several conclusions that I have in this dissertation. An example on how to read and digest the following pages is provided.

For example, in section $B$, the mathematical fluid is subject to non-zero gravity $(G=0.333)$, stabilizing surface tension $(S=100)$, destabilizing thermocapillarity $(M=35.1)$. It is non evaporating $(E=0.0)$ and the effect of disjoining pressures is neglected $(A=0.0)$. The film is subject to a cosine initial condition whose amplitude is $5 \%$ of the non-dimensional initial film thickness $\left(h_{0}=1\right.$, amplitude $=$ $0.05)(1+0.05[\cos (2 \pi x / L)+\sin (2 \pi x / L)] \cos (2 \pi y / L))$. The rupture time is recorded for completion.

The plots show a film profile for the initial condition and a contorted film profile at rupture. The discrete Fourier transform plots that accompany the initial condition and rupture profile show the wavelength trends as the film is led to rupture due to thermocapillarity.

The different initial conditions used are:

1. Cosine initial condition:

$$
1+0.05[\cos (2 \pi x / L)+\sin (2 \pi x / L)] \cos (2 \pi y / L)
$$

2. Sine initial condition:

$$
1+0.05[\cos (2 \pi x / L)+\sin (2 \pi x / L)] \sin (2 \pi y / L)
$$

3. Cosine $\times$ Sine:

$$
1+0.05[\cos (2 \pi x / L)+\sin (2 \pi x / L)] \sin (2 \pi y / L) \cos (2 \pi y / L)
$$

4. Cosine-squared:

$$
1+0.05[\cos (2 \pi x / L)+\sin (2 \pi x / L)](\cos (2 \pi y / L))^{2}
$$

5. Cosine-rotated:

$$
1-0.05(\cos (2 \pi(x+y) / \sqrt{2} L)+\sin (2 \pi(x+y) / \sqrt{2} L)) \cos (2 \pi(x-y) / \sqrt{2} L)
$$

6. Cosine-Y:

$$
1-0.05 \cos (2 \pi y) / L)
$$


Non Evaporating Mathematical fluid, $\mathrm{G}=0.333$, Cosine initial condition, 


\begin{tabular}{|l|l|}
\hline$G$ & 0.333 \\
$S$ & 100 \\
$A$ & 0 \\
$E$ & 0 \\
$M$ & 35.1 \\
$\mathrm{Pr}$ & 7.02 \\
$R a$ & 0 \\
Domain size & 1 \\
Wave number & 1 \\
Amplitude of perturbation & 5 \\
Rupture time & 8706.7 \\
Initial condition & Cosine initial condition \\
\hline
\end{tabular}

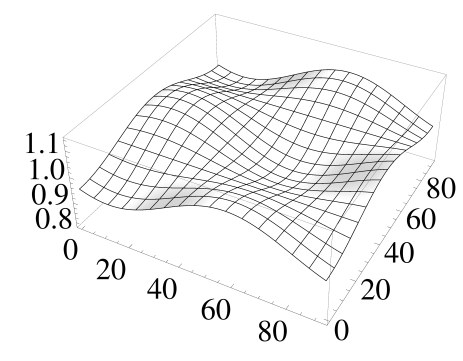

Figure 2.1. Initial condition

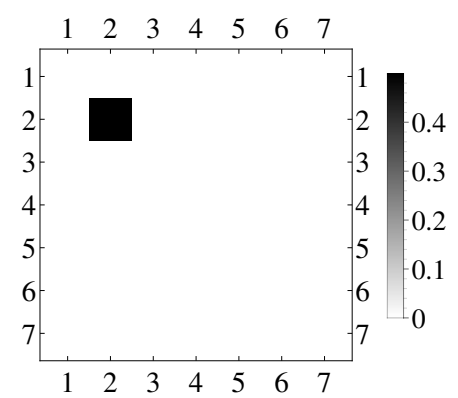

Figure 2.3. DFT, Initial condition

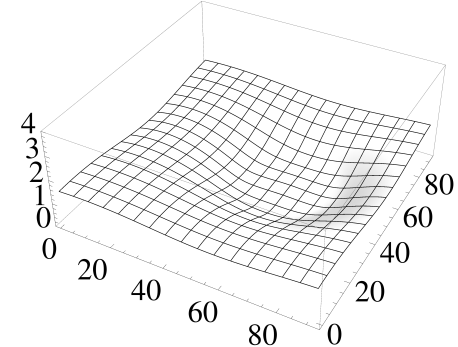

Figure 2.2. Rupture

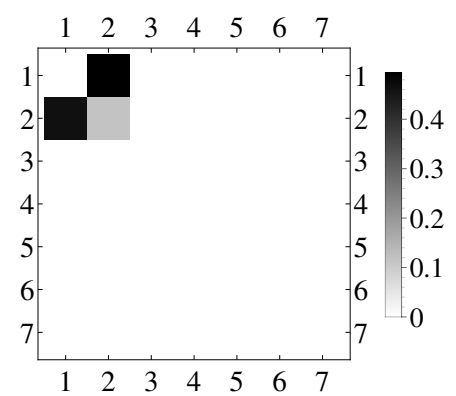

Figure 2.4. DFT, rupture 


\begin{tabular}{|l|l|}
\hline$G$ & 0.333 \\
$S$ & 100 \\
$A$ & 0 \\
$E$ & 0 \\
$M$ & 35.1 \\
$\mathrm{Pr}$ & 7.02 \\
$R a$ & 0 \\
Domain size & 1 \\
Wave number & 1 \\
Amplitude of perturbation & 5 \\
Rupture time & 8200. \\
Initial condition & Cosine initial condition \\
\hline
\end{tabular}

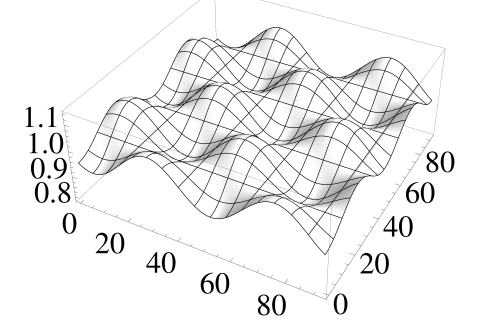

Figure 2.5. Initial condition

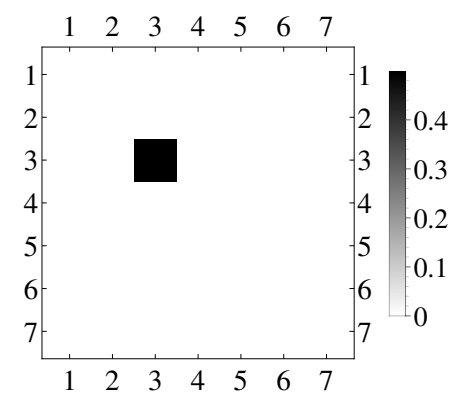

Figure 2.7. DFT, Initial condition

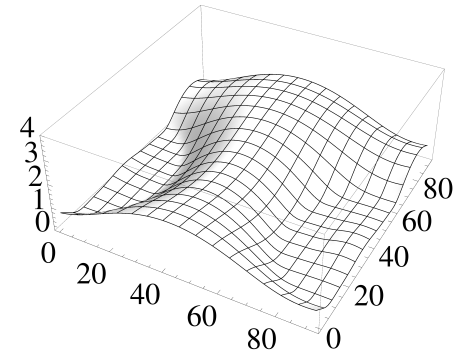

Figure 2.6. Rupture

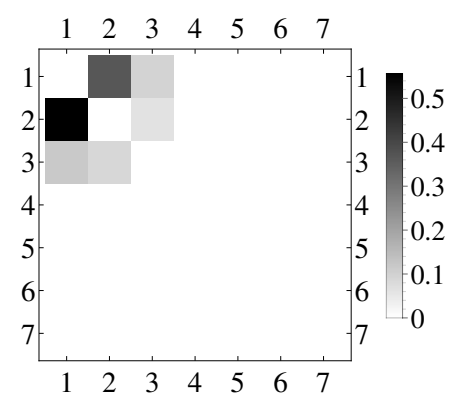

Figure 2.8. DFT, rupture 


\begin{tabular}{|l|l|}
\hline$G$ & 0.333 \\
$S$ & 100 \\
$A$ & 0 \\
$E$ & 0 \\
$M$ & 35.1 \\
$\mathrm{Pr}$ & 7.02 \\
$R a$ & 0 \\
Domain size & 1 \\
Wave number & 2 \\
Amplitude of perturbation & 5 \\
Rupture time & 8335.6 \\
Initial condition & Cosine initial condition \\
\hline
\end{tabular}

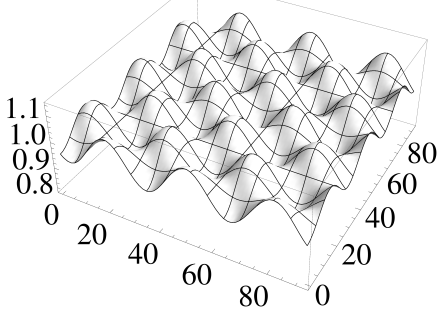

Figure 2.9. Initial condition

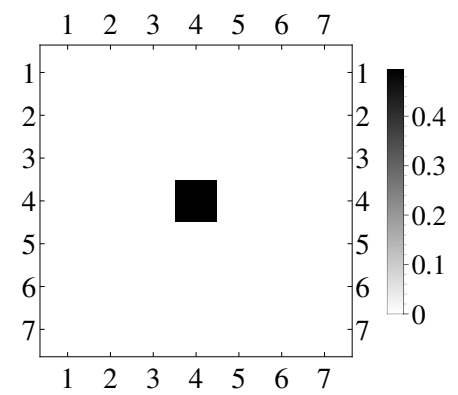

Figure 2.11. DFT, Initial condition

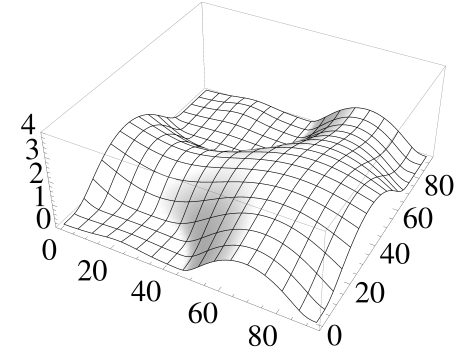

Figure 2.10. Rupture

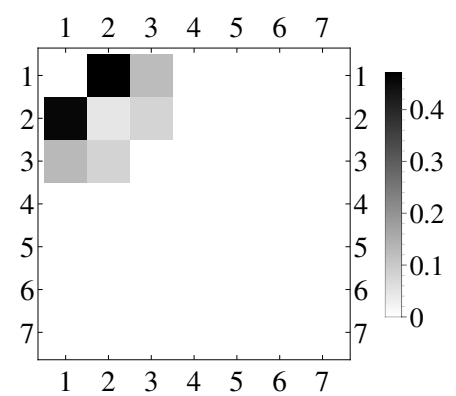

Figure 2.12. DFT, rupture 


\begin{tabular}{|l|l|}
\hline$G$ & 0 \\
$S$ & 100 \\
$A$ & 0 \\
$E$ & 0 \\
$M$ & 35.1 \\
$\mathrm{Pr}$ & 7.02 \\
$R a$ & 0 \\
Domain size & 2 \\
Wave number & 1 \\
Amplitude of perturbation & 5 \\
Rupture time & 1879.7 \\
Initial condition & Cosine initial condition \\
\hline
\end{tabular}

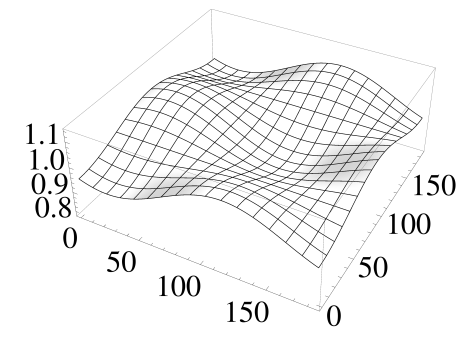

Figure 2.13. Initial condition

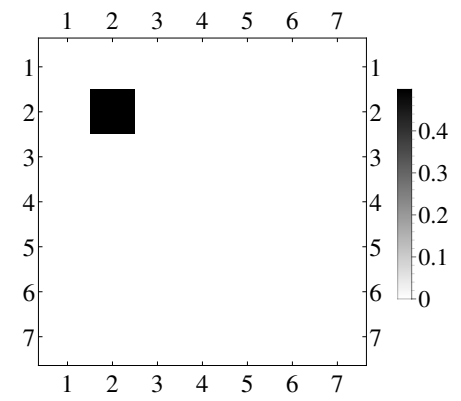

Figure 2.15. DFT, Initial condition

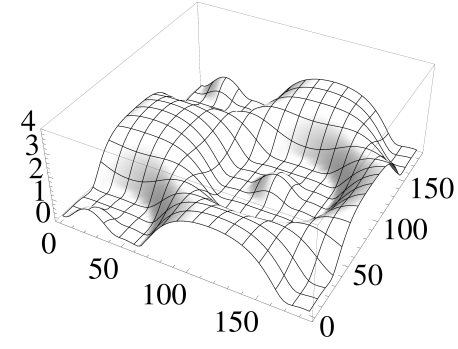

Figure 2.14. Rupture

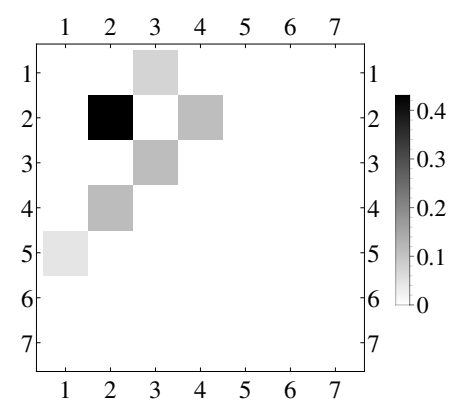

Figure 2.16. DFT, rupture 


\begin{tabular}{|l|l|}
\hline$G$ & 0.333 \\
$S$ & 100 \\
$A$ & 0 \\
$E$ & 0 \\
$M$ & 35.1 \\
$\mathrm{Pr}$ & 7.02 \\
$R a$ & 0 \\
Domain size & 2 \\
Wave number & 1 \\
Amplitude of perturbation & 5 \\
Rupture time & 1879.7 \\
Initial condition & Cosine initial condition \\
\hline
\end{tabular}

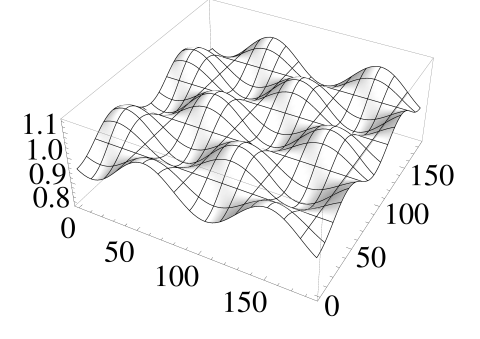

Figure 2.17. Initial condition

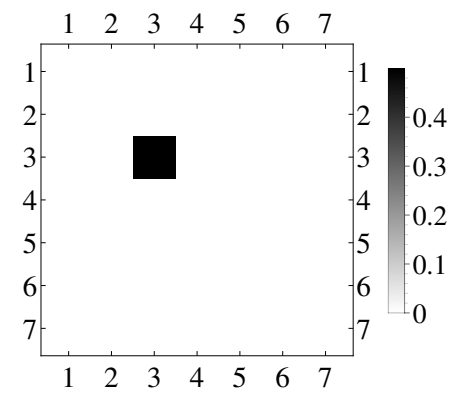

Figure 2.19. DFT, Initial condition

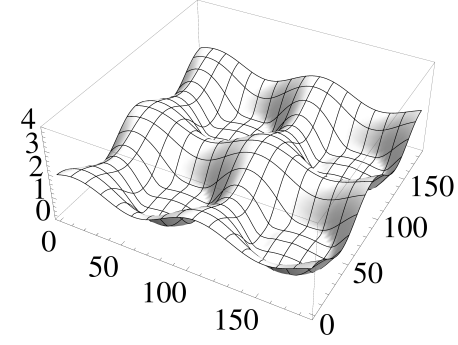

Figure 2.18. Rupture

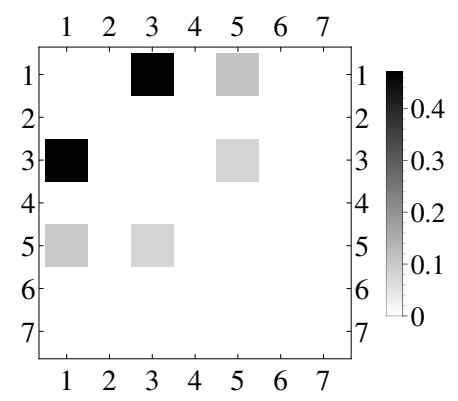

Figure 2.20. DFT, rupture 


\begin{tabular}{|l|l|}
\hline$G$ & 0.333 \\
$S$ & 100 \\
$A$ & 0 \\
$E$ & 0 \\
$M$ & 35.1 \\
$\mathrm{Pr}$ & 7.02 \\
$R a$ & 0 \\
Domain size & 2 \\
Wave number & 2 \\
Amplitude of perturbation & 5 \\
Rupture time & 8695.6 \\
Initial condition & Cosine initial condition \\
\hline
\end{tabular}

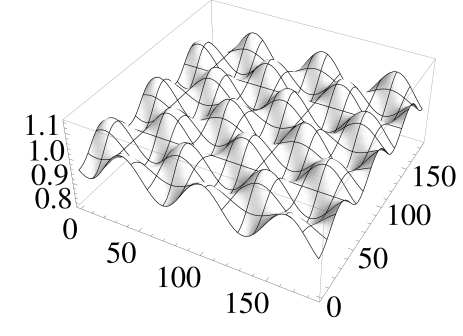

Figure 2.21. Initial condition

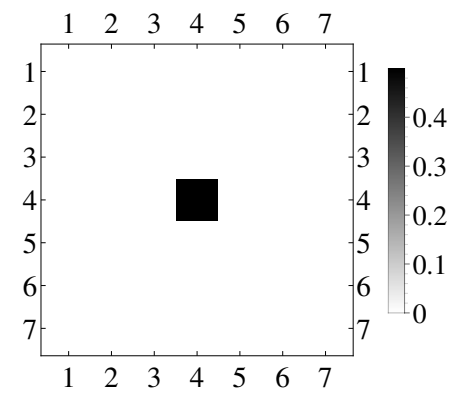

Figure 2.23. DFT, Initial condition

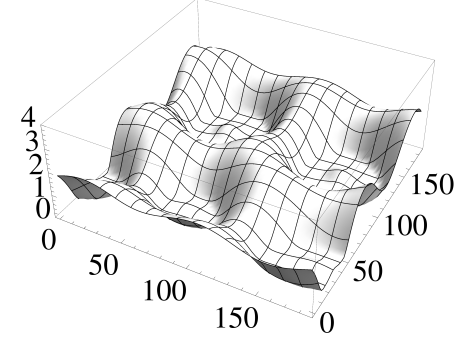

Figure 2.22. Rupture

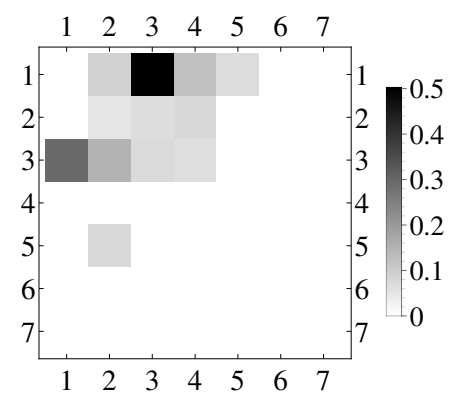

Figure 2.24. DFT, rupture 


\begin{tabular}{|l|l|}
\hline$G$ & 0.333 \\
$S$ & 100 \\
$A$ & 0 \\
$E$ & 0 \\
$M$ & 35.1 \\
$\mathrm{Pr}$ & 7.02 \\
$R a$ & 0 \\
Domain size & 2 \\
Wave number & 3 \\
Amplitude of perturbation & 5 \\
Rupture time & 9034.2 \\
Initial condition & Cosine initial condition \\
\hline
\end{tabular}

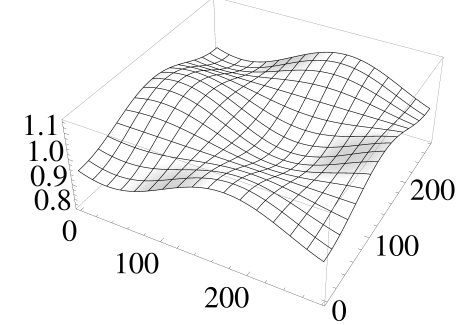

Figure 2.25. Initial condition

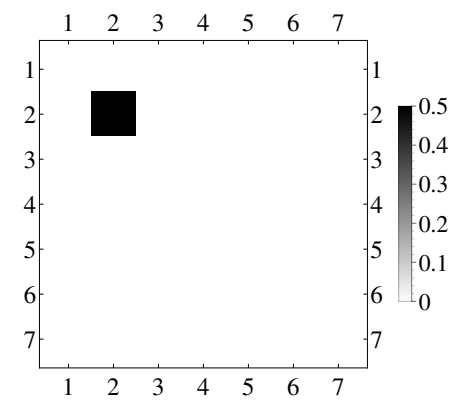

Figure 2.27. DFT, Initial condition

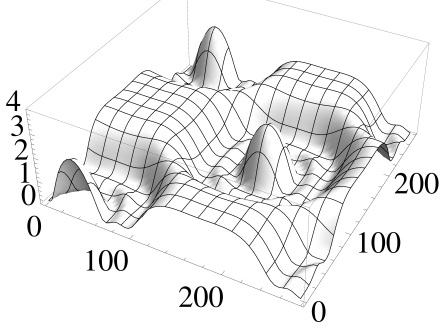

Figure 2.26. Rupture

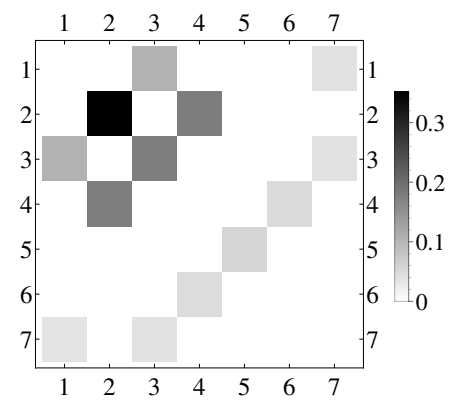

Figure 2.28. DFT, rupture 


\begin{tabular}{|l|l|}
\hline$G$ & 0.333 \\
$S$ & 100 \\
$A$ & 0 \\
$E$ & 0 \\
$M$ & 35.1 \\
$\mathrm{Pr}$ & 7.02 \\
$R a$ & 0 \\
Domain size & 3 \\
Wave number & 1 \\
Amplitude of perturbation & 5 \\
Rupture time & 3104.1 \\
Initial condition & Cosine initial condition \\
\hline
\end{tabular}

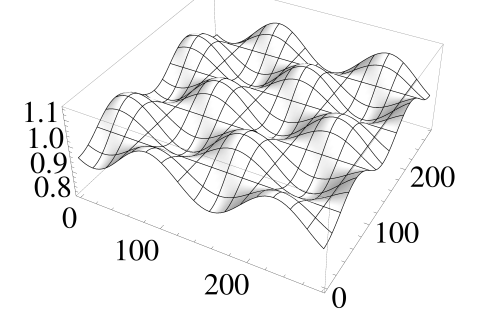

Figure 2.29. Initial condition

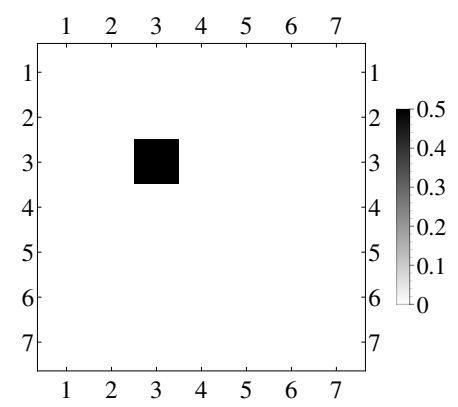

Figure 2.31. DFT, Initial condition

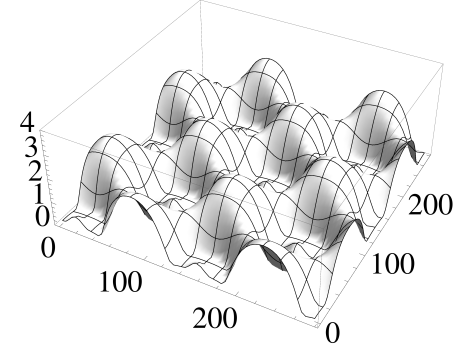

Figure 2.30. Rupture

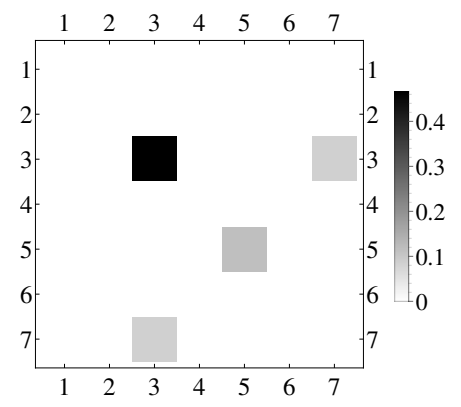

Figure 2.32. DFT, rupture 


\begin{tabular}{|l|l|}
\hline$G$ & 0.333 \\
$S$ & 100 \\
$A$ & 0 \\
$E$ & 0 \\
$M$ & 35.1 \\
$\mathrm{Pr}$ & 7.02 \\
$R a$ & 0 \\
Domain size & 3 \\
Wave number & 2 \\
Amplitude of perturbation & 5 \\
Rupture time & 1432.3 \\
Initial condition & Cosine initial condition \\
\hline
\end{tabular}

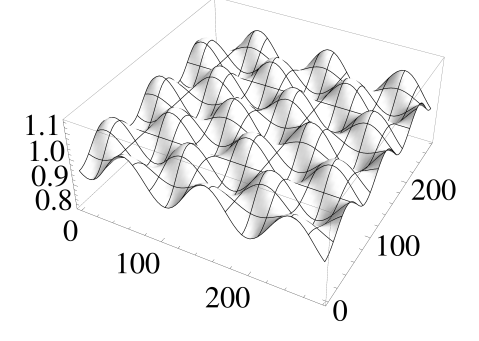

Figure 2.33. Initial condition

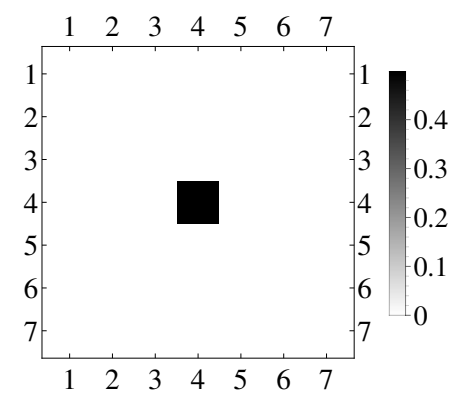

Figure 2.35. DFT, Initial condition

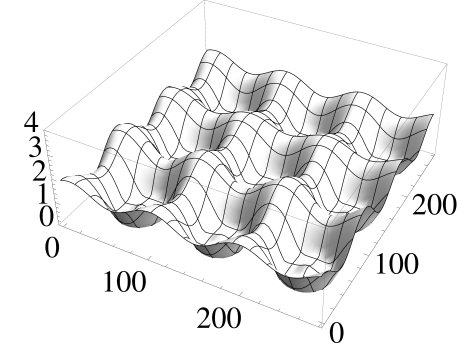

Figure 2.34. Rupture

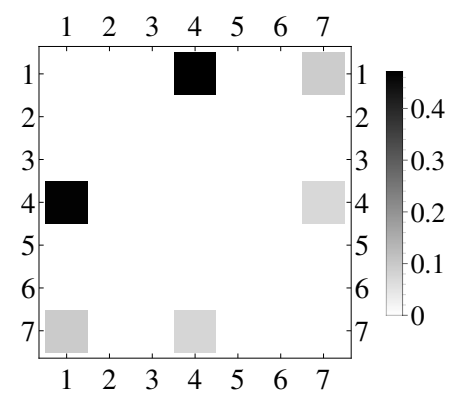

Figure 2.36. DFT, rupture 
Non Evaporating Mathematical fluid, $G=0.0$, Cosine initial condition 


\begin{tabular}{|l|l|}
\hline$G$ & 0.0 \\
$S$ & 100 \\
$A$ & 0 \\
$E$ & 0 \\
$M$ & 35.1 \\
$\mathrm{Pr}$ & 7.02 \\
$R a$ & 0 \\
Domain size & 2 \\
Wave number & 3 \\
Amplitude of perturbation & 5 \\
Rupture time & 8775. \\
Initial condition & Cosine initial condition \\
\hline
\end{tabular}

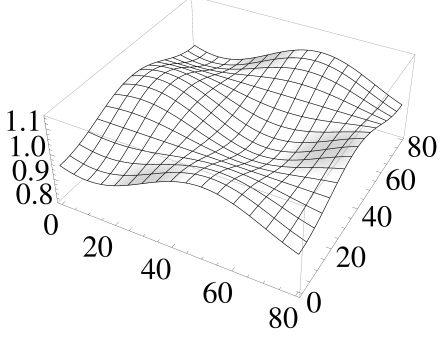

Figure 2.37. Initial condition

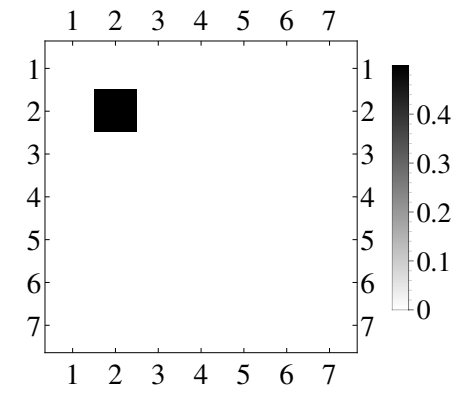

Figure 2.39. DFT, Initial condition

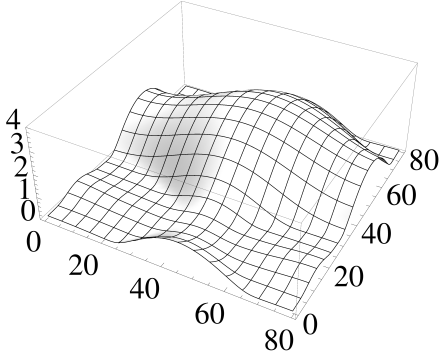

Figure 2.38. Rupture

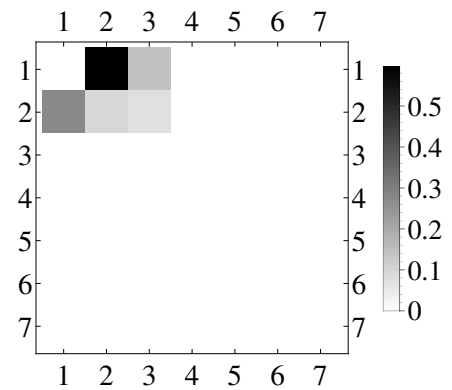

Figure 2.40. DFT, rupture 


\begin{tabular}{|l|l|}
\hline$G$ & 0 \\
$S$ & 100 \\
$A$ & 0 \\
$E$ & 0 \\
$M$ & 35.1 \\
$\operatorname{Pr}$ & 7.02 \\
$R a$ & 0 \\
Domain size & 1 \\
Wave number & 1 \\
Amplitude of perturbation & 5 \\
Rupture time & 3350.1 \\
Initial condition & Cosine initial condition \\
\hline
\end{tabular}

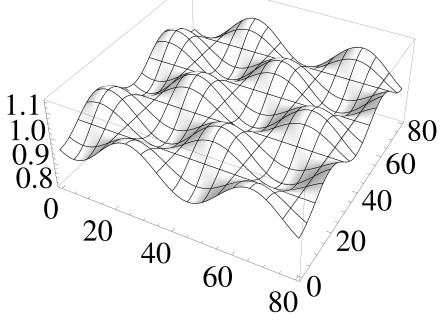

Figure 2.41. Initial condition

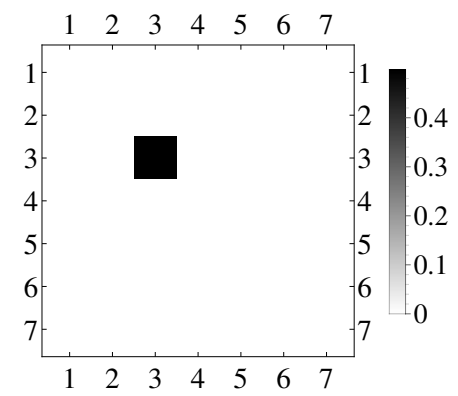

Figure 2.43. DFT, Initial condition

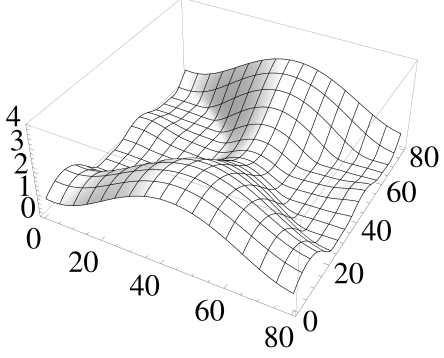

Figure 2.42. Rupture

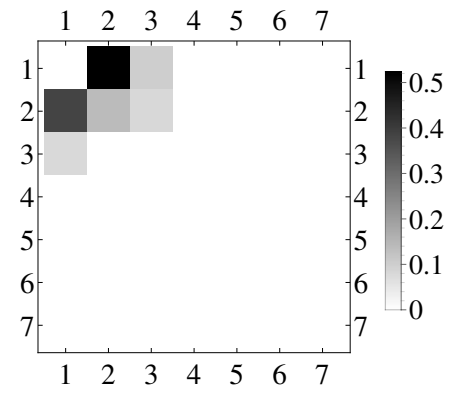

Figure 2.44. DFT, rupture 


\begin{tabular}{|l|l|}
\hline$G$ & 0.0 \\
$S$ & 100 \\
$A$ & 0 \\
$E$ & 0 \\
$M$ & 35.1 \\
$\mathrm{Pr}$ & 7.02 \\
$R a$ & 0 \\
Domain size & 2 \\
Wave number & 3 \\
Amplitude of perturbation & 5 \\
Rupture time & 8701.1 \\
Initial condition & Cosine initial condition \\
\hline
\end{tabular}

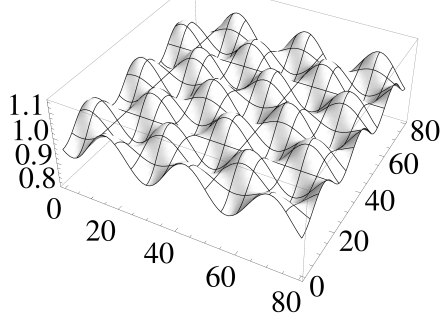

Figure 2.45. Initial condition

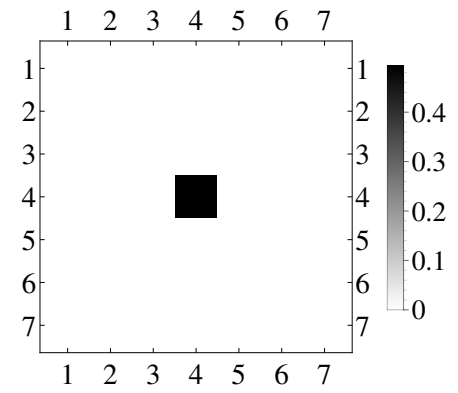

Figure 2.47. DFT, Initial condition

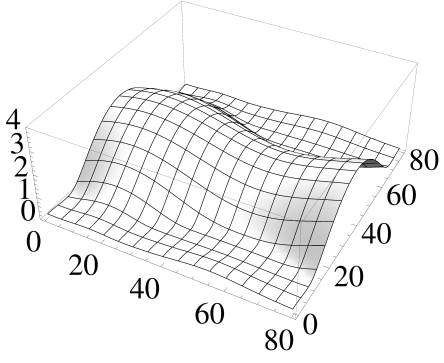

Figure 2.46. Rupture

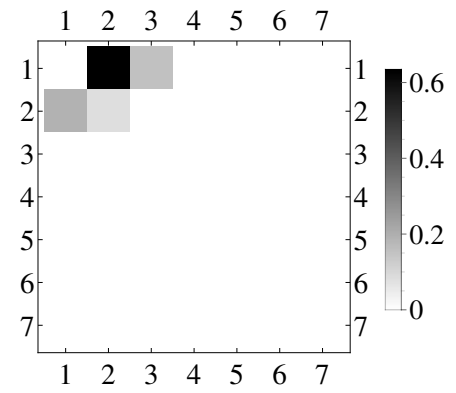

Figure 2.48. DFT, rupture 


\begin{tabular}{|l|l|}
\hline$G$ & 0. \\
$S$ & 100 \\
$A$ & 0 \\
$E$ & 0 \\
$M$ & 35.1 \\
$\mathrm{Pr}$ & 7.02 \\
$R a$ & 0 \\
Domain size & 2 \\
Wave number & 1 \\
Amplitude of perturbation & 5 \\
Rupture time & 1264.1 \\
Initial condition & Cosine initial condition \\
\hline
\end{tabular}
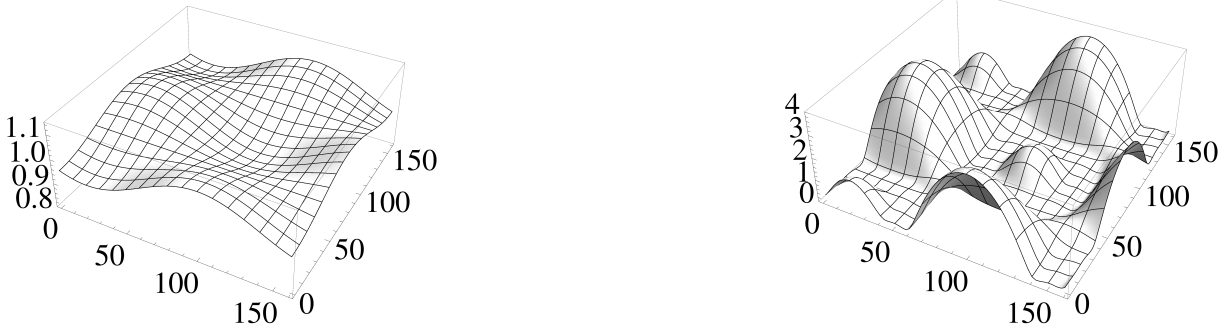

Figure 2.49. Initial condition

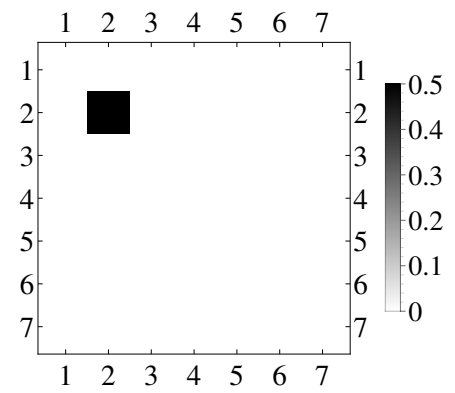

Figure 2.50. Rupture

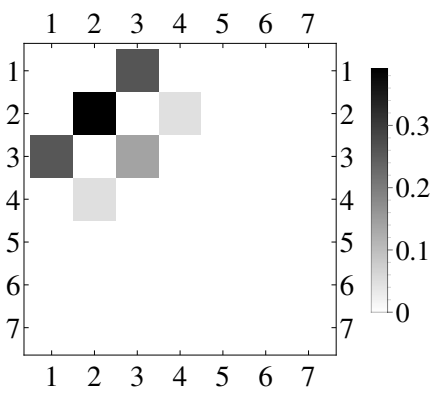

Figure 2.51. DFT, Initial condition

Figure 2.52. DFT, rupture 


\begin{tabular}{|l|l|}
\hline$G$ & 0. \\
$S$ & 100 \\
$A$ & 0 \\
$E$ & 0 \\
$M$ & 35.1 \\
$\mathrm{Pr}$ & 7.02 \\
$R a$ & 0 \\
Domain size & 2 \\
Wave number & 2 \\
Amplitude of perturbation & 5 \\
Rupture time & 4431. \\
Initial condition & Cosine initial condition \\
\hline
\end{tabular}

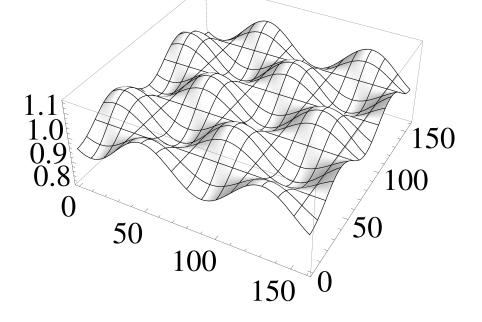

Figure 2.53. Initial condition

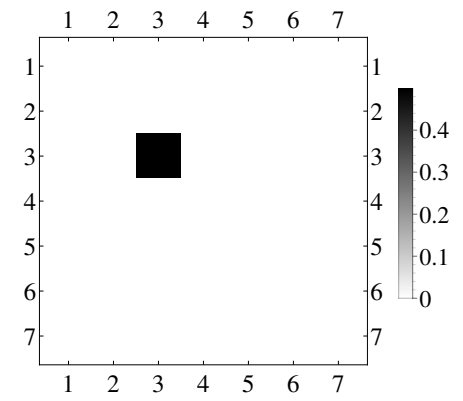

Figure 2.55. DFT, Initial condition

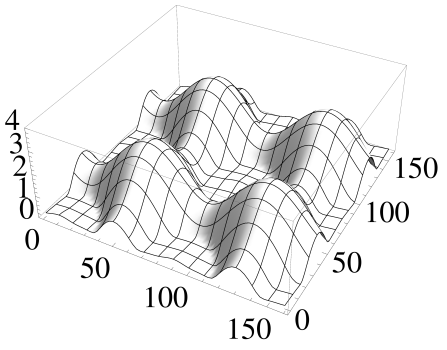

Figure 2.54. Rupture

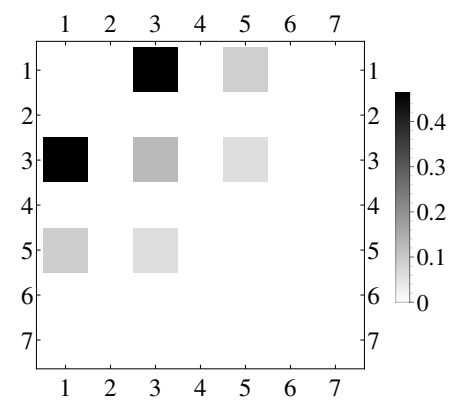

Figure 2.56. DFT, rupture 


\begin{tabular}{|l|l|}
\hline$G$ & 0. \\
$S$ & 100 \\
$A$ & 0 \\
$E$ & 0 \\
$M$ & 35.1 \\
$\mathrm{Pr}$ & 7.02 \\
$R a$ & 0 \\
Domain size & 2 \\
Wave number & 3 \\
Amplitude of perturbation & 5 \\
Rupture time & 4406.6 \\
Initial condition & Cosine initial condition \\
\hline
\end{tabular}

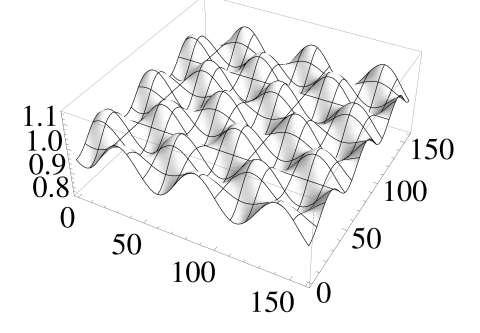

Figure 2.57. Initial condition

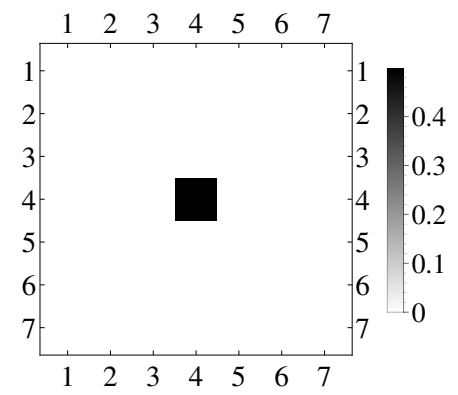

Figure 2.59. DFT, Initial condition

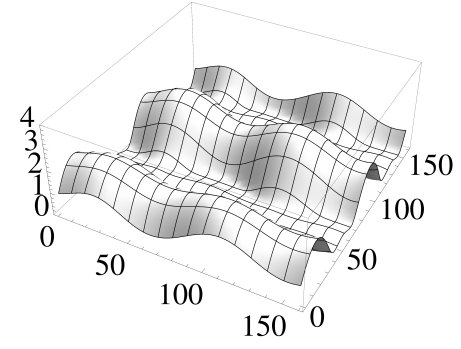

Figure 2.58. Rupture

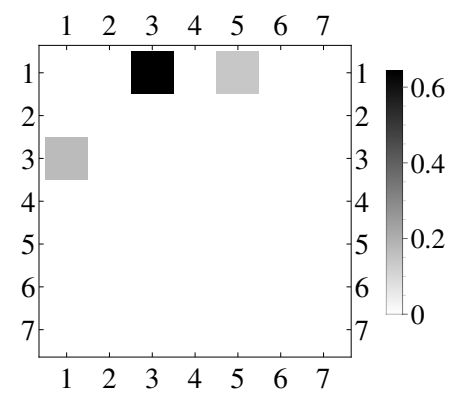

Figure 2.60. DFT, rupture 


\begin{tabular}{|l|l|}
\hline$G$ & 0 \\
$S$ & 100 \\
$A$ & 0 \\
$E$ & 0 \\
$M$ & 35.1 \\
$\mathrm{Pr}$ & 7.02 \\
$R a$ & 0 \\
Domain size & 3 \\
Wave number & 1 \\
Amplitude of perturbation & 5 \\
Rupture time & 1753.3 \\
Initial condition & Cosine initial condition \\
\hline
\end{tabular}
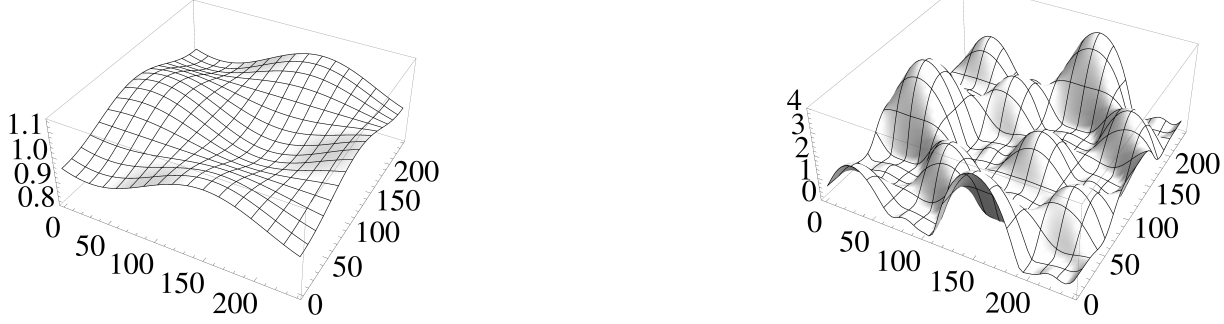

Figure 2.61. Initial condition

Figure 2.62. Rupture
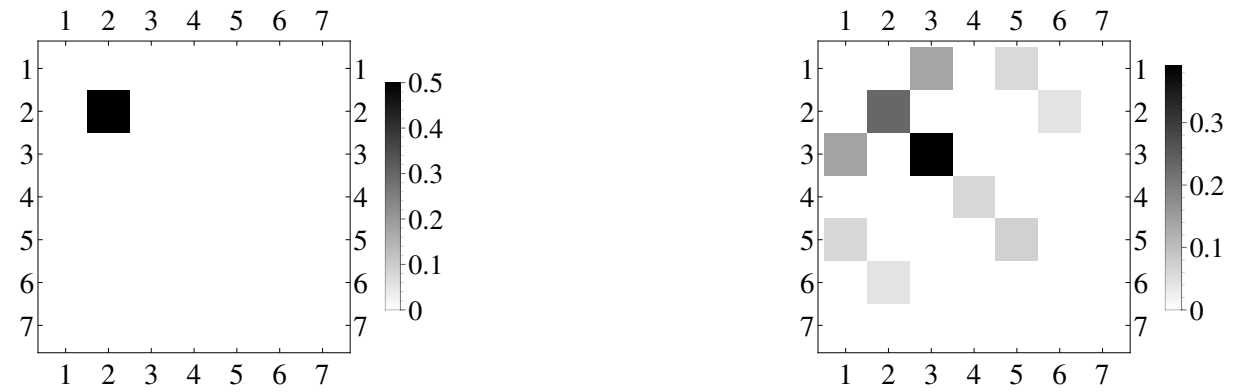

Figure 2.63. DFT, Initial condition

Figure 2.64. DFT, rupture 


\begin{tabular}{|l|l|}
\hline$G$ & 0 \\
$S$ & 100 \\
$A$ & 0 \\
$E$ & 0 \\
$M$ & 35.1 \\
$\mathrm{Pr}$ & 7.02 \\
$R a$ & 0 \\
Domain size & 3 \\
Wave number & 2 \\
Amplitude of perturbation & 5 \\
Rupture time & 945.87 \\
Initial condition & Cosine initial condition \\
\hline
\end{tabular}

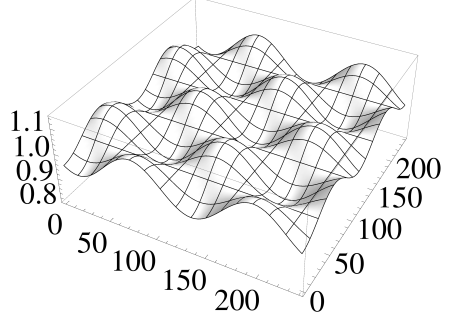

Figure 2.65. Initial condition

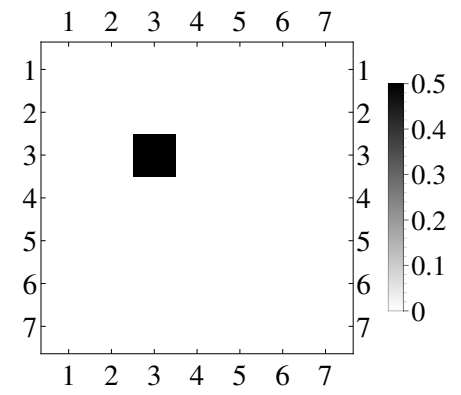

Figure 2.67. DFT, Initial condition

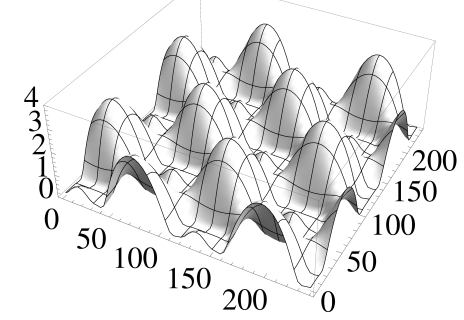

Figure 2.66. Rupture

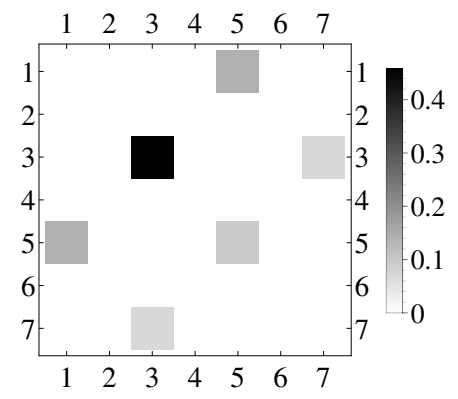

Figure 2.68. DFT, rupture 


\begin{tabular}{|l|l|}
\hline$G$ & 0 \\
$S$ & 100 \\
$A$ & 0 \\
$E$ & 0 \\
$M$ & 35.1 \\
$\mathrm{Pr}$ & 7.02 \\
$R a$ & 0 \\
Domain size & 3 \\
Wave number & 3 \\
Amplitude of perturbation & 5 \\
Rupture time & 4543.5 \\
Initial condition & Cosine initial condition \\
\hline
\end{tabular}

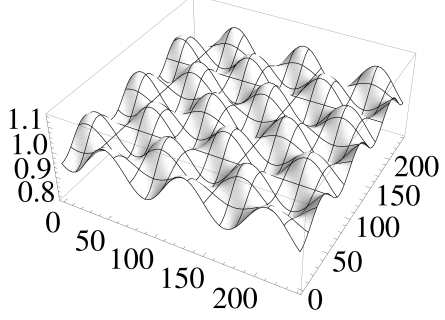

Figure 2.69. Initial condition

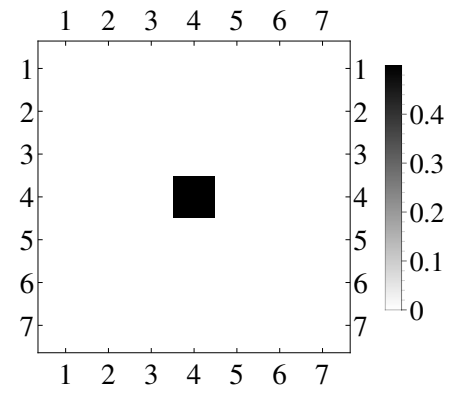

Figure 2.71. DFT, Initial condition

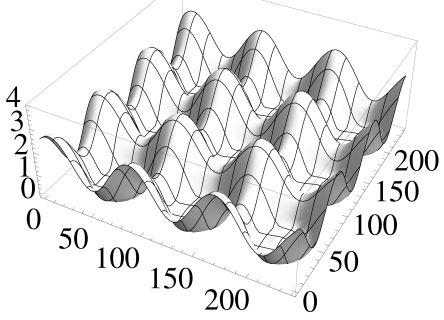

Figure 2.70. Rupture

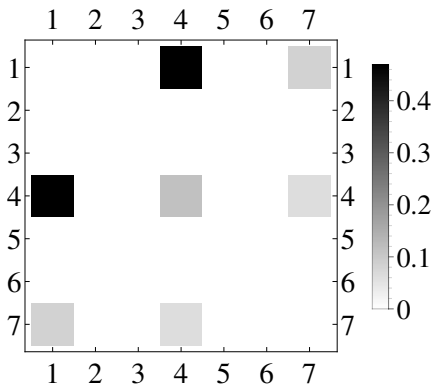

Figure 2.72. DFT, rupture 
Slowly Evaporating Mathematical fluid, $\mathrm{E}=0.0001, \mathrm{G}=0.0$, Cosine initial condition 


\begin{tabular}{|l|l|}
\hline$G$ & 0 \\
$S$ & 100 \\
$A$ & 0 \\
$E$ & 0.0001 \\
$M$ & 35.1 \\
$\mathrm{Pr}$ & 7.02 \\
$R a$ & 0 \\
Domain size & 1 \\
Wave number & 1 \\
Amplitude of perturbation & 5 \\
Rupture time & 1565.3 \\
Initial condition & Cosine initial condition \\
\hline
\end{tabular}

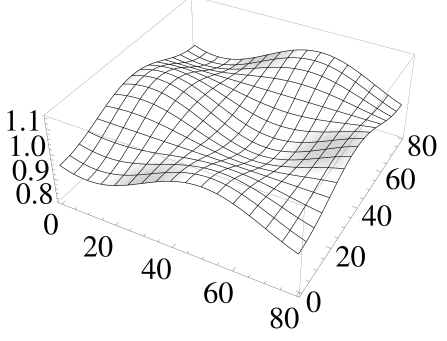

Figure 2.73. Initial condition

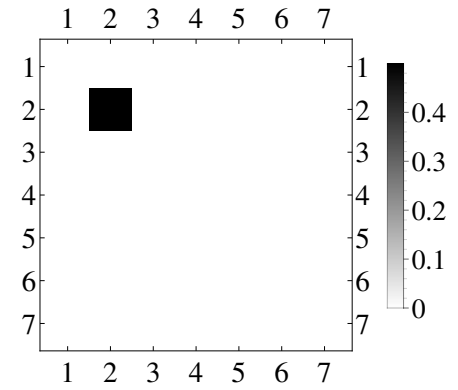

Figure 2.75. DFT, Initial condition

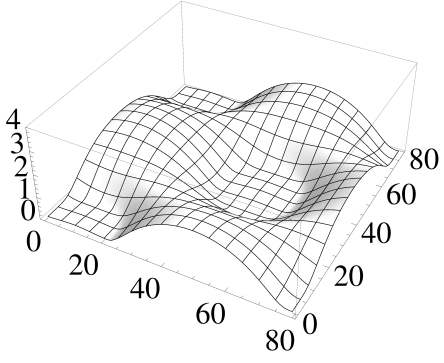

Figure 2.74. Rupture

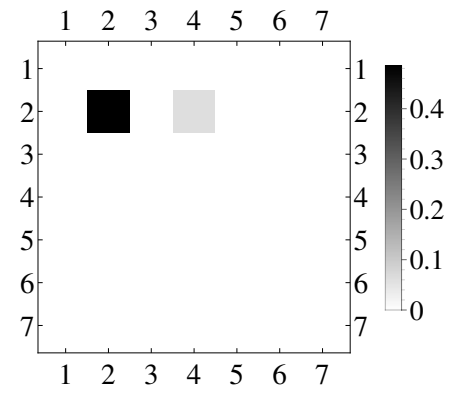

Figure 2.76. DFT, rupture 


\begin{tabular}{|l|l|}
\hline$G$ & 0 \\
$S$ & 100 \\
$A$ & 0 \\
$E$ & 0.0001 \\
$M$ & 35.1 \\
$\mathrm{Pr}$ & 7.02 \\
$R a$ & 0 \\
Domain size & 1 \\
Wave number & 2 \\
Amplitude of perturbation & 5 \\
Rupture time & 2912.5 \\
Initial condition & Cosine initial condition \\
\hline
\end{tabular}

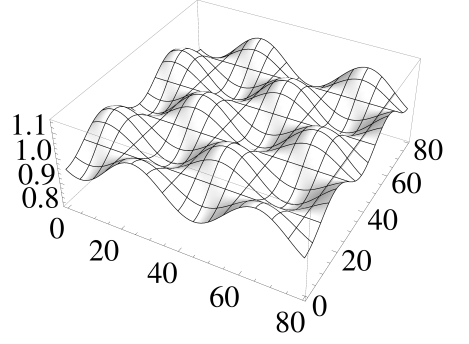

Figure 2.77. Initial condition

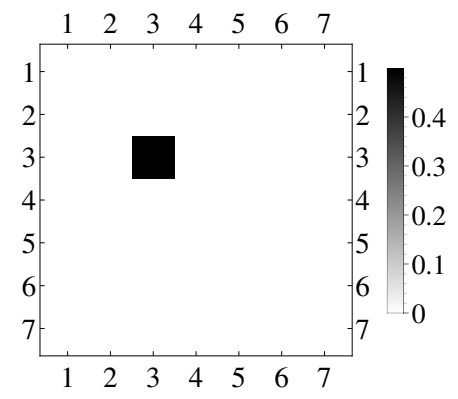

Figure 2.79. DFT, Initial condition

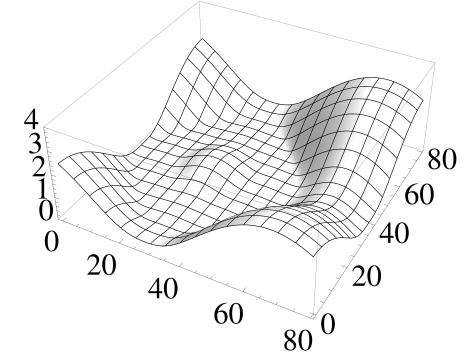

Figure 2.78. Rupture

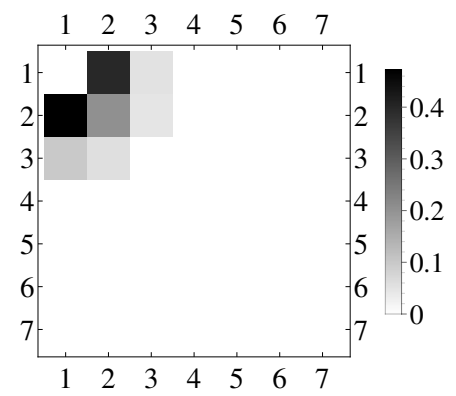

Figure 2.80. DFT, rupture 


\begin{tabular}{|l|l|}
\hline$G$ & 0 \\
$S$ & 100 \\
$A$ & 0 \\
$E$ & 0.0001 \\
$M$ & 35.1 \\
$\mathrm{Pr}$ & 7.02 \\
$R a$ & 0 \\
Domain size & 1 \\
Wave number & 3 \\
Amplitude of perturbation & 5 \\
Rupture time & 3041.2 \\
Initial condition & Cosine initial condition \\
\hline
\end{tabular}

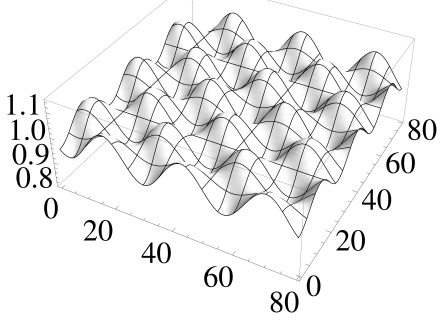

Figure 2.81. Initial condition

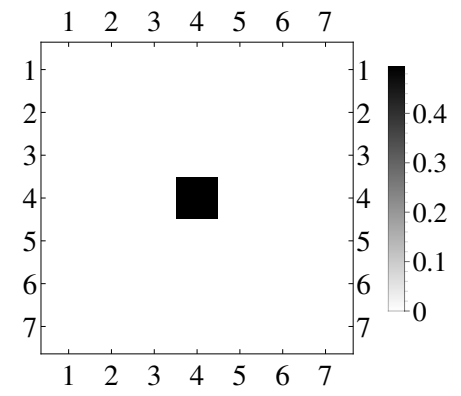

Figure 2.83. DFT, Initial condition

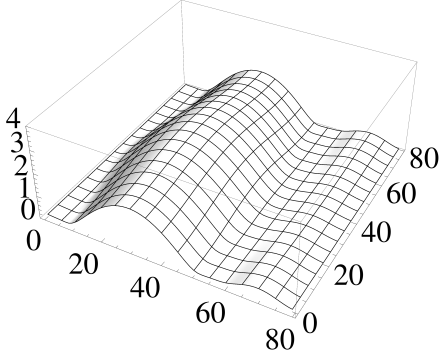

Figure 2.82. Rupture

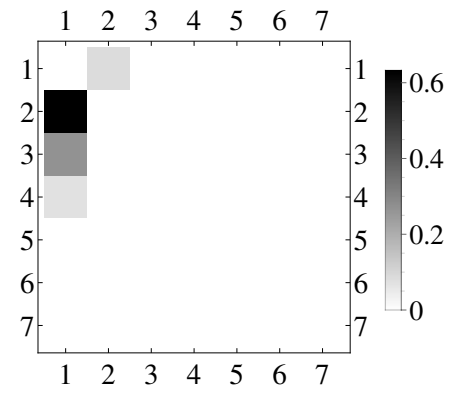

Figure 2.84. DFT, rupture 


\begin{tabular}{|l|l|}
\hline$G$ & 0. \\
$S$ & 100 \\
$A$ & 0 \\
$E$ & 0.0001 \\
$M$ & 35.1 \\
$\mathrm{Pr}$ & 7.02 \\
$R a$ & 0 \\
Domain size & 2 \\
Wave number & 1 \\
Amplitude of perturbation & 5 \\
Rupture time & 1207. \\
Initial condition & Cosine initial condition \\
\hline
\end{tabular}
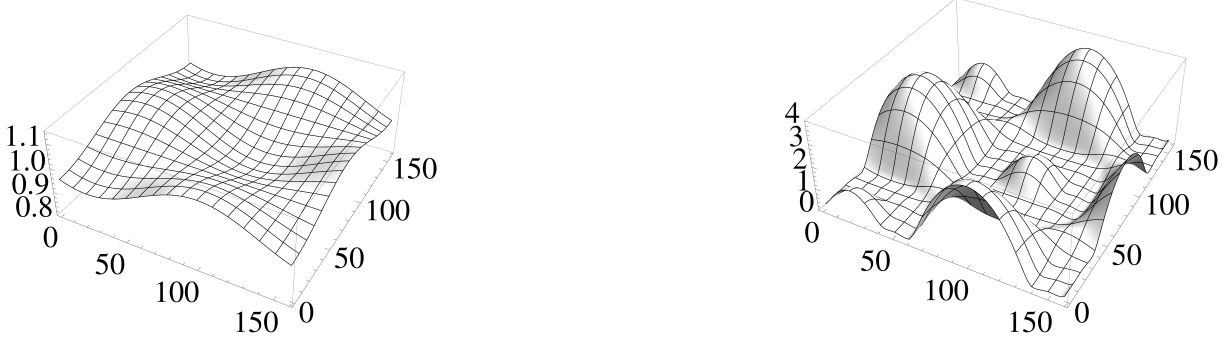

Figure 2.85. Initial condition

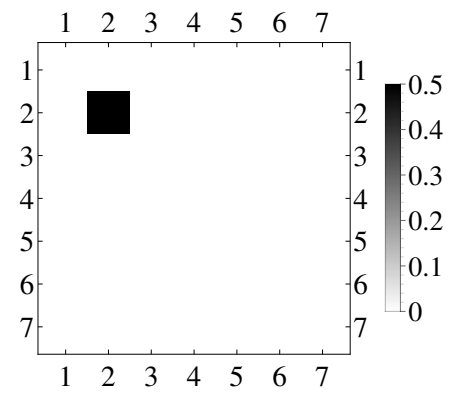

Figure 2.86. Rupture

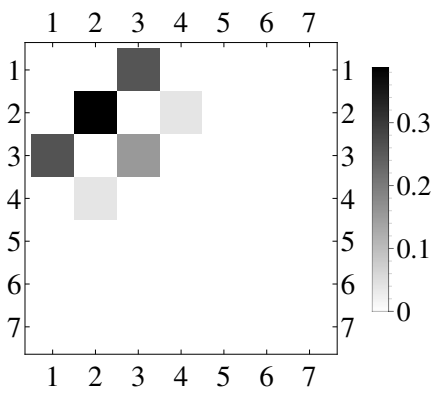

Figure 2.87. DFT, Initial condition

Figure 2.88. DFT, rupture 


\begin{tabular}{|l|l|}
\hline$G$ & 0. \\
$S$ & 100 \\
$A$ & 0 \\
$E$ & 0.0001 \\
$M$ & 35.1 \\
$\mathrm{Pr}$ & 7.02 \\
$R a$ & 0 \\
Domain size & 2 \\
Wave number & 2 \\
Amplitude of perturbation & 5 \\
Rupture time & 1581.2 \\
Initial condition & Cosine initial condition \\
\hline
\end{tabular}

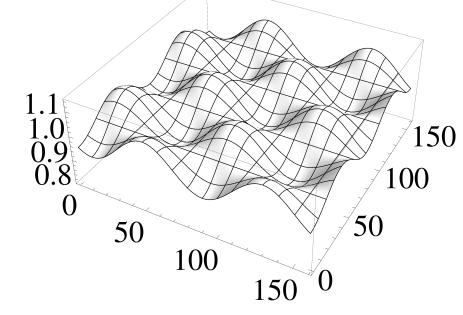

Figure 2.89. Initial condition

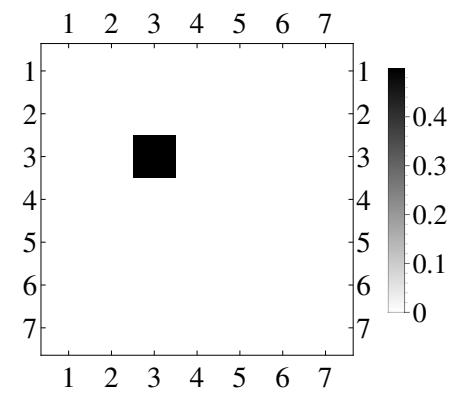

Figure 2.91. DFT, Initial condition

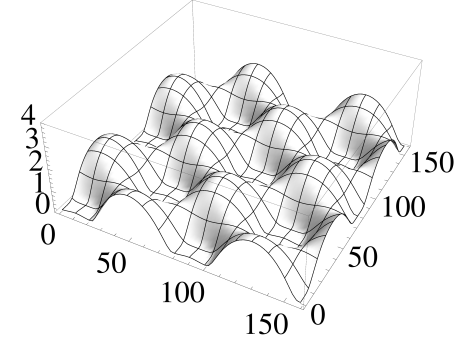

Figure 2.90. Rupture

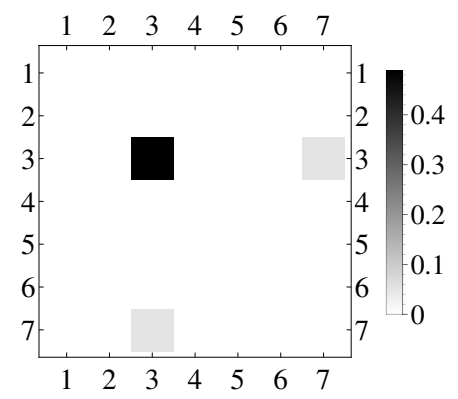

Figure 2.92. DFT, rupture 


\begin{tabular}{|l|l|}
\hline$G$ & 0. \\
$S$ & 100 \\
$A$ & 0 \\
$E$ & 0.0001 \\
$M$ & 35.1 \\
$\mathrm{Pr}$ & 7.02 \\
$R a$ & 0 \\
Domain size & 2 \\
Wave number & 3 \\
Amplitude of perturbation & 5 \\
Rupture time & 4362.8 \\
Initial condition & Cosine initial condition \\
\hline
\end{tabular}

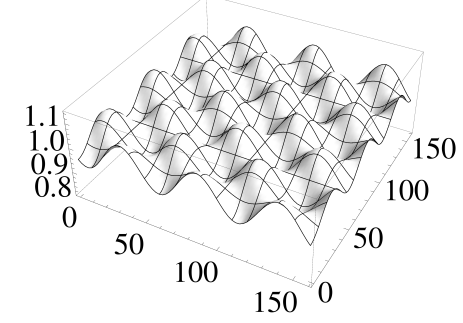

Figure 2.93. Initial condition

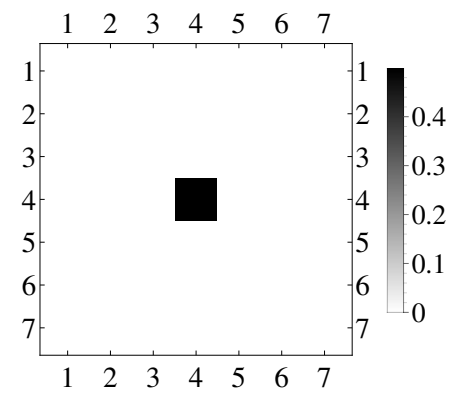

Figure 2.95. DFT, Initial condition

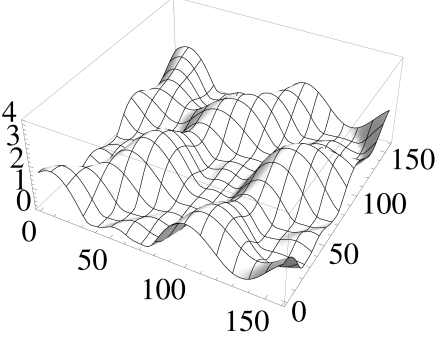

Figure 2.94. Rupture

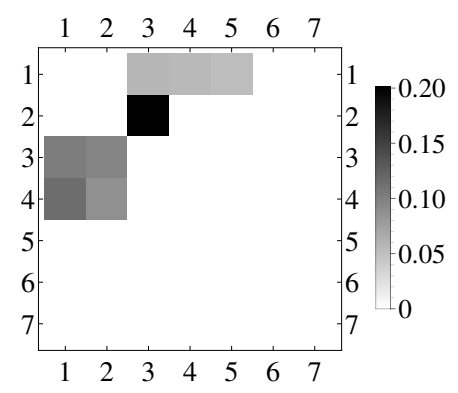

Figure 2.96. DFT, rupture 


\begin{tabular}{|l|l|}
\hline$G$ & 0 \\
$S$ & 100 \\
$A$ & 0 \\
$E$ & 0.0001 \\
$M$ & 35.1 \\
$\mathrm{Pr}$ & 7.02 \\
$R a$ & 0 \\
Domain size & 3 \\
Wave number & 1 \\
Amplitude of perturbation & 5 \\
Rupture time & 1663. \\
Initial condition & Cosine initial condition \\
\hline
\end{tabular}

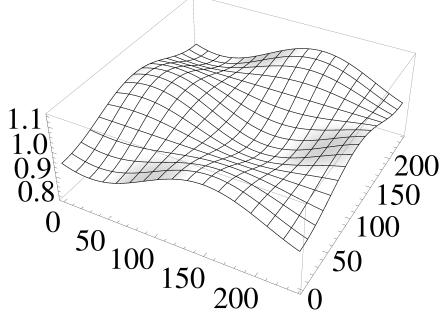

Figure 2.97. Initial condition

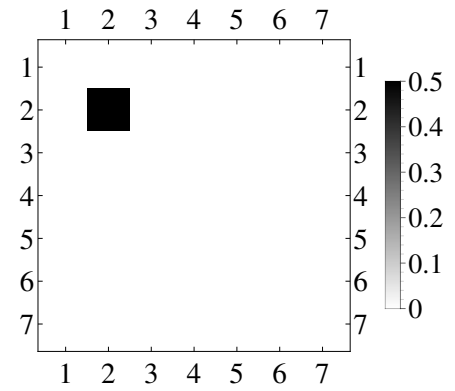

Figure 2.99. DFT, Initial condition

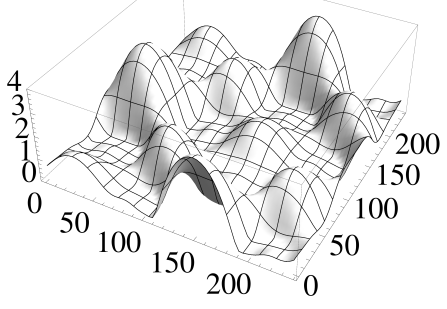

Figure 2.98. Rupture

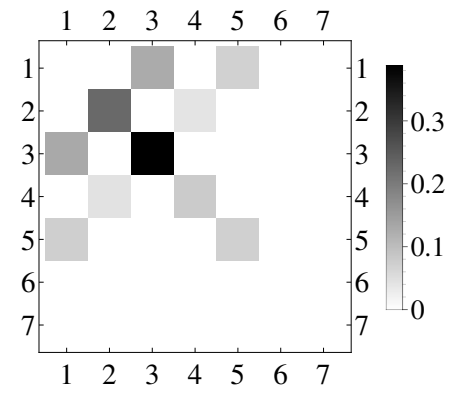

Figure 2.100. DFT, rupture 


\begin{tabular}{|l|l|}
\hline$G$ & 0 \\
$S$ & 100 \\
$A$ & 0 \\
$E$ & 0.0001 \\
$M$ & 35.1 \\
$\mathrm{Pr}$ & 7.02 \\
$R a$ & 0 \\
Domain size & 3 \\
Wave number & 2 \\
Amplitude of perturbation & 5 \\
Rupture time & 909.27 \\
Initial condition & Cosine initial condition \\
\hline
\end{tabular}

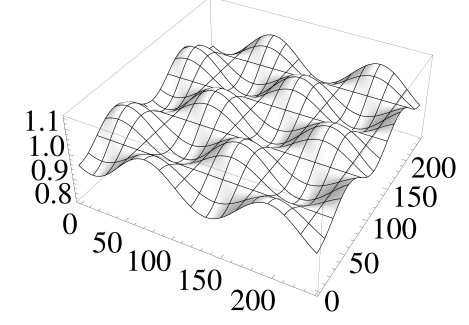

Figure 2.101. Initial condition

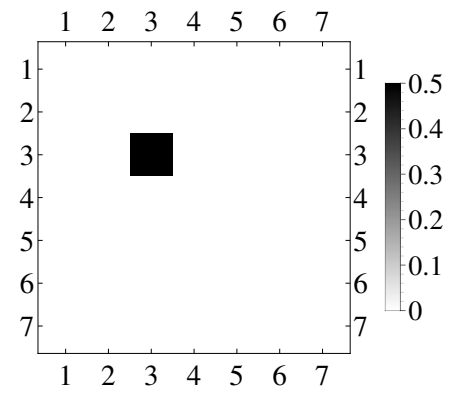

Figure 2.103. DFT, Initial condition

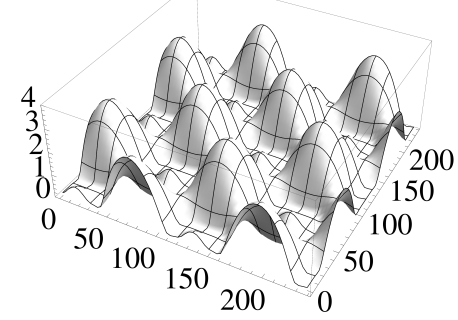

Figure 2.102. Rupture

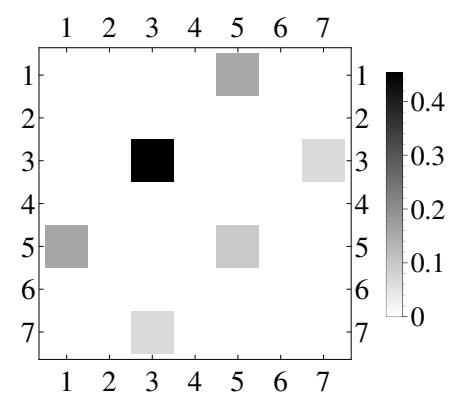

Figure 2.104. DFT, rupture 


\begin{tabular}{|l|l|}
\hline$G$ & 0 \\
$S$ & 100 \\
$A$ & 0 \\
$E$ & 0.0001 \\
$M$ & 35.1 \\
$\mathrm{Pr}$ & 7.02 \\
$R a$ & 0 \\
Domain size & 3 \\
Wave number & 3 \\
Amplitude of perturbation & 5 \\
Rupture time & 1583. \\
Initial condition & Cosine initial condition \\
\hline
\end{tabular}

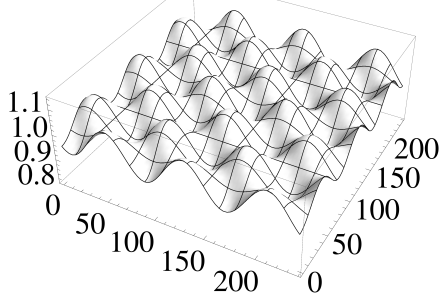

Figure 2.105. Initial condition

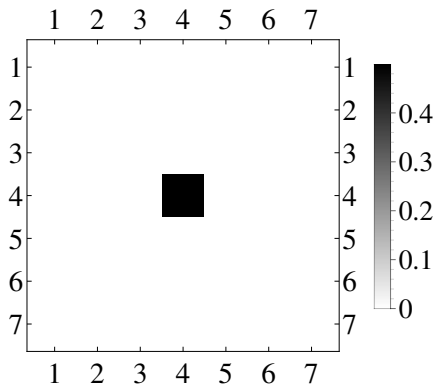

Figure 2.107. DFT, Initial condition

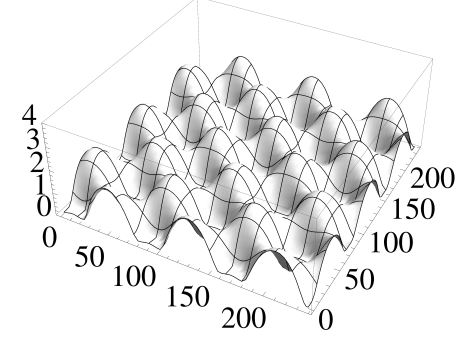

Figure 2.106. Rupture

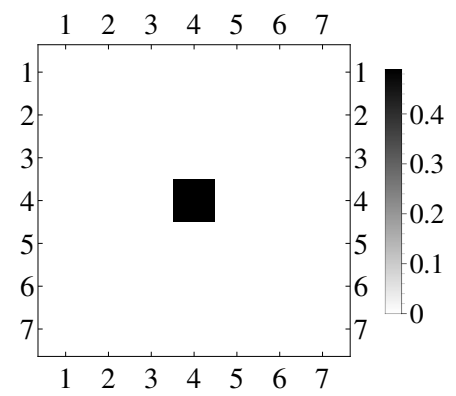

Figure 2.108. DFT, rupture 


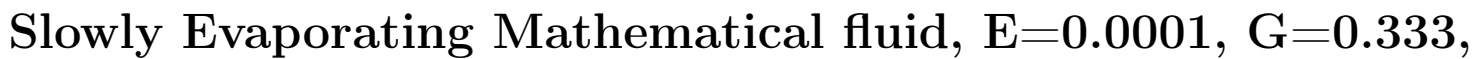
Cosine initial condition 


\begin{tabular}{|l|l|}
\hline$G$ & 0.333 \\
$S$ & 100 \\
$A$ & 0 \\
$E$ & 0.0001 \\
$M$ & 35.1 \\
$\mathrm{Pr}$ & 7.02 \\
$R a$ & 0 \\
Domain size & 1 \\
Wave number & 1 \\
Amplitude of perturbation & 5 \\
Rupture time & 1849.1 \\
Initial condition & Cosine initial condition \\
\hline
\end{tabular}

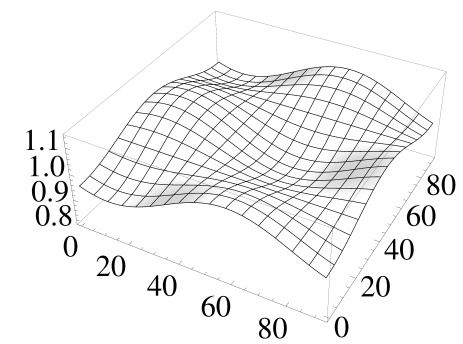

Figure 2.109. Initial condition

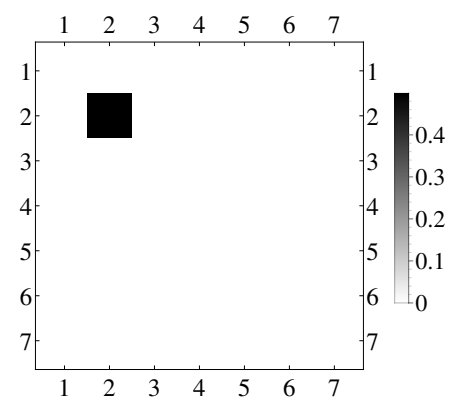

Figure 2.111. DFT, Initial condition

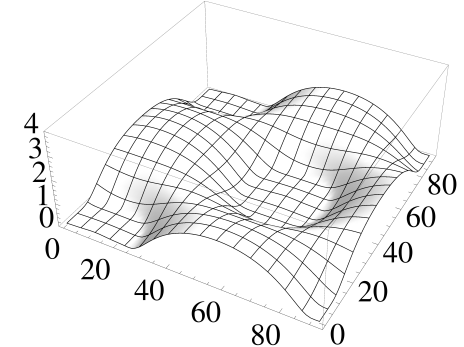

Figure 2.110. Rupture

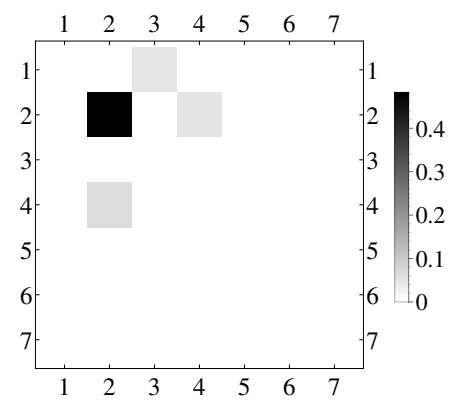

Figure 2.112. DFT, rupture 


\begin{tabular}{|l|l|}
\hline$G$ & 0.333 \\
$S$ & 100 \\
$A$ & 0 \\
$E$ & 0.0001 \\
$M$ & 35.1 \\
$\mathrm{Pr}$ & 7.02 \\
$R a$ & 0 \\
Domain size & 1 \\
Wave number & 2 \\
Amplitude of perturbation & 5 \\
Rupture time & 6798.8 \\
Initial condition & Cosine initial condition \\
\hline
\end{tabular}

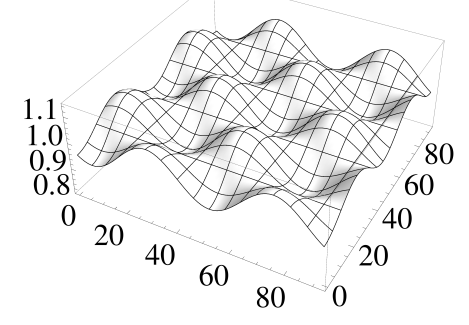

Figure 2.113. Initial condition

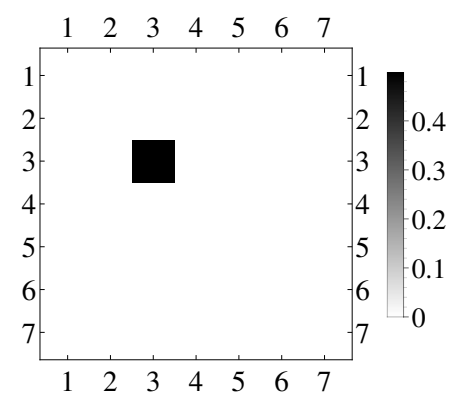

Figure 2.115. DFT, Initial condition

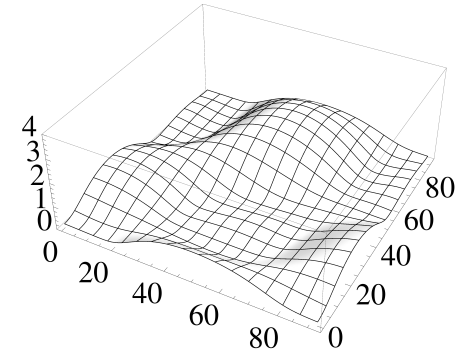

Figure 2.114. Rupture

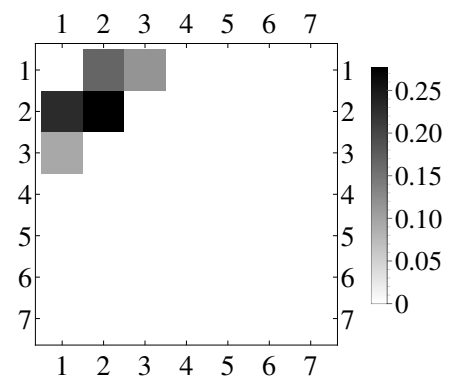

Figure 2.116. DFT, rupture 


\begin{tabular}{|l|l|}
\hline$G$ & 0.333 \\
$S$ & 100 \\
$A$ & 0 \\
$E$ & 0.0001 \\
$M$ & 35.1 \\
$\mathrm{Pr}$ & 7.02 \\
$R a$ & 0 \\
Domain size & 1 \\
Wave number & 3 \\
Amplitude of perturbation & 5 \\
Rupture time & 6774.1 \\
Initial condition & Cosine initial condition \\
\hline
\end{tabular}

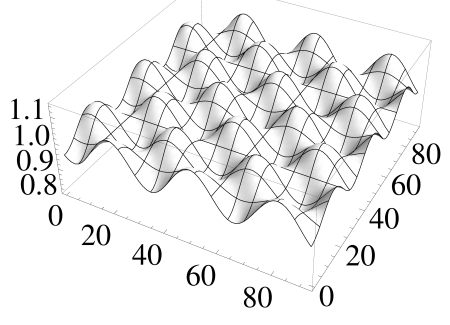

Figure 2.117. Initial condition

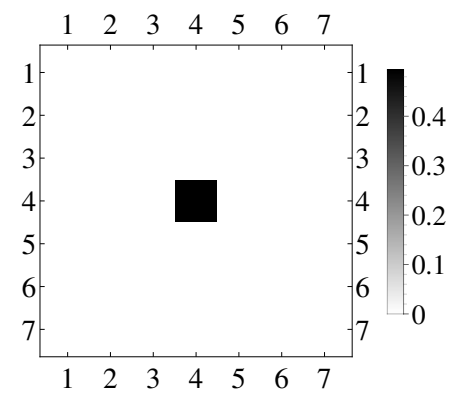

Figure 2.119. DFT, Initial condition

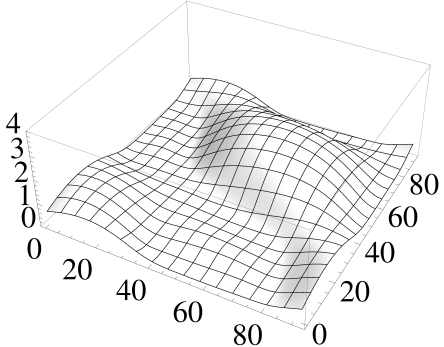

Figure 2.118. Rupture

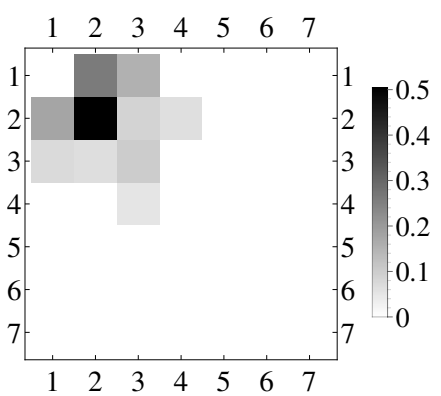

Figure 2.120. DFT, rupture 


\begin{tabular}{|l|l|}
\hline$G$ & 0.333 \\
$S$ & 100 \\
$A$ & 0 \\
$E$ & 0.0001 \\
$M$ & 35.1 \\
$\mathrm{Pr}$ & 7.02 \\
$R a$ & 0 \\
Domain size & 2 \\
Wave number & 1 \\
Amplitude of perturbation & 5 \\
Rupture time & 1906. \\
Initial condition & Cosine initial condition \\
\hline
\end{tabular}

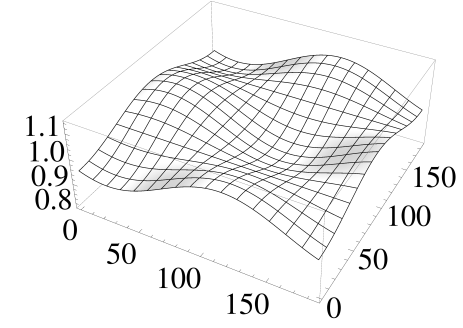

Figure 2.121. Initial condition

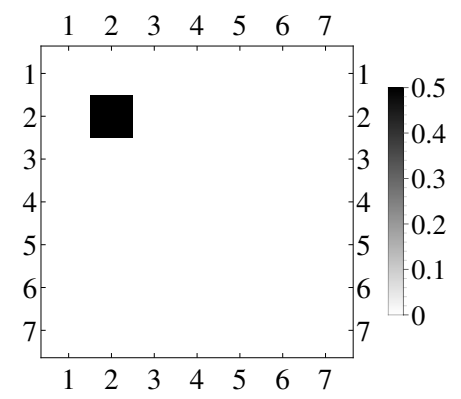

Figure 2.123. DFT, Initial condition

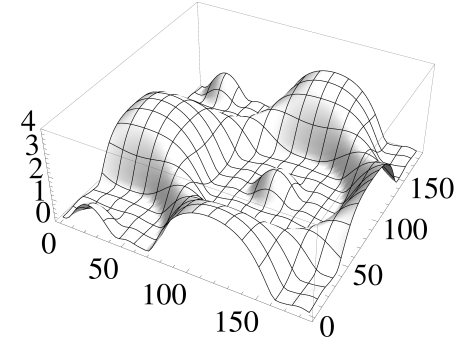

Figure 2.122. Rupture

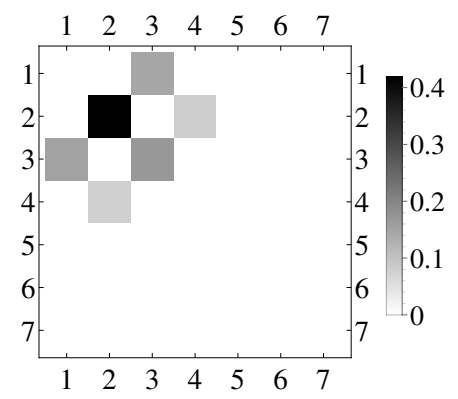

Figure 2.124. DFT, rupture 


\begin{tabular}{|l|l|}
\hline$G$ & 0.333 \\
$S$ & 100 \\
$A$ & 0 \\
$E$ & 0.0001 \\
$M$ & 35.1 \\
$\mathrm{Pr}$ & 7.02 \\
$R a$ & 0 \\
Domain size & 2 \\
Wave number & 2 \\
Amplitude of perturbation & 5 \\
Rupture time & 1846.6 \\
Initial condition & Cosine initial condition \\
\hline
\end{tabular}

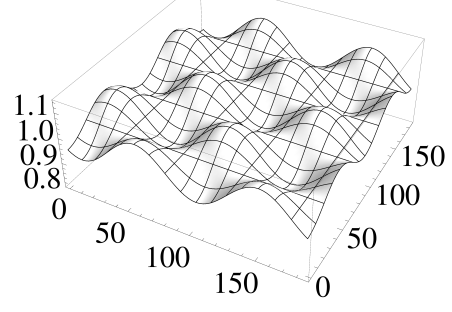

Figure 2.125. Initial condition

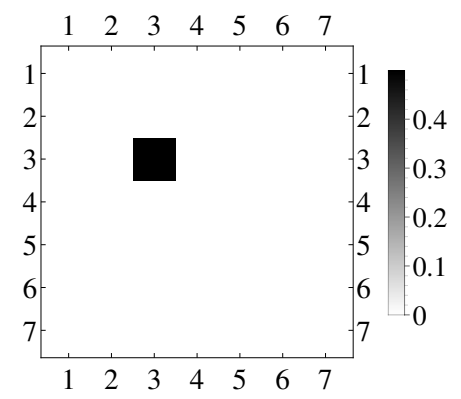

Figure 2.127. DFT, Initial condition

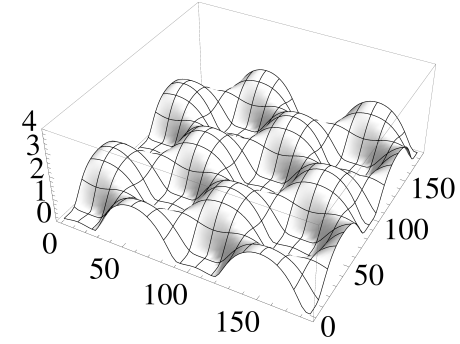

Figure 2.126. Rupture

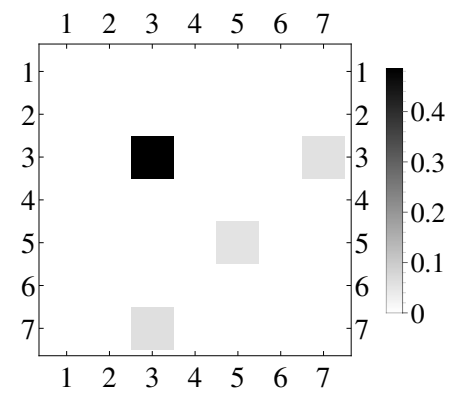

Figure 2.128. DFT, rupture 


\begin{tabular}{|l|l|}
\hline$G$ & 0.333 \\
$S$ & 100 \\
$A$ & 0 \\
$E$ & 0.0001 \\
$M$ & 35.1 \\
$\mathrm{Pr}$ & 7.02 \\
$R a$ & 0 \\
Domain size & 2 \\
Wave number & 3 \\
Amplitude of perturbation & 5 \\
Rupture time & 6696.3 \\
Initial condition & Cosine initial condition \\
\hline
\end{tabular}

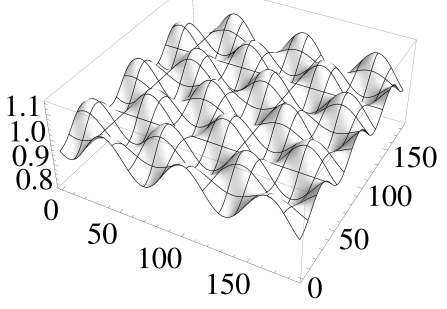

Figure 2.129. Initial condition

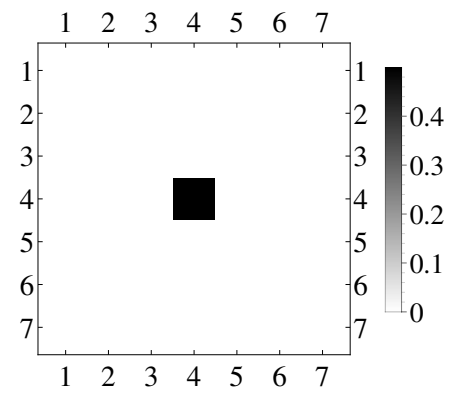

Figure 2.131. DFT, Initial condition

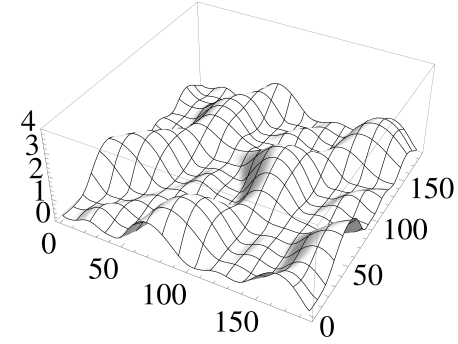

Figure 2.130. Rupture

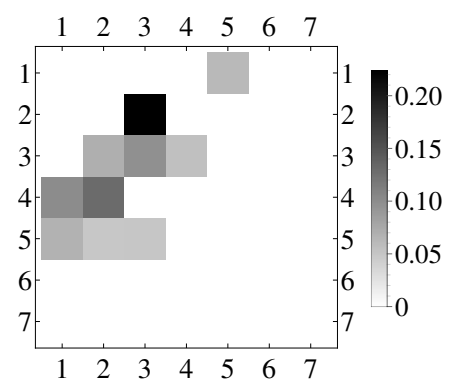

Figure 2.132. DFT, rupture 


\begin{tabular}{|l|l|}
\hline$G$ & 0.333 \\
$S$ & 100 \\
$A$ & 0 \\
$E$ & 0.0001 \\
$M$ & 35.1 \\
$\mathrm{Pr}$ & 7.02 \\
$R a$ & 0 \\
Domain size & 3 \\
Wave number & 1 \\
Amplitude of perturbation & 5 \\
Rupture time & 2785.5 \\
Initial condition & Cosine initial condition \\
\hline
\end{tabular}

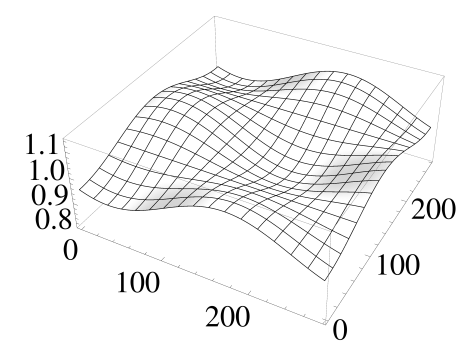

Figure 2.133. Initial condition

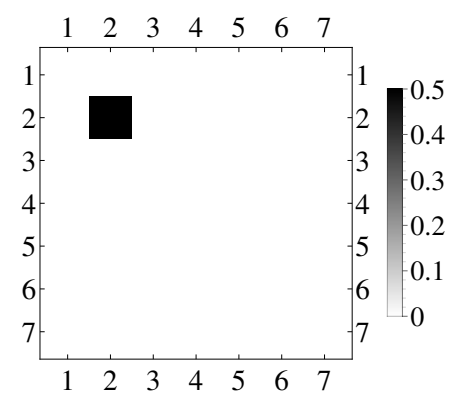

Figure 2.135. DFT, Initial condition

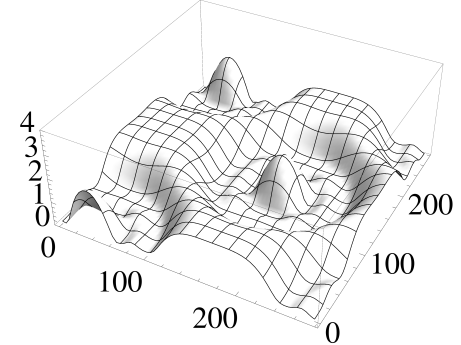

Figure 2.134. Rupture

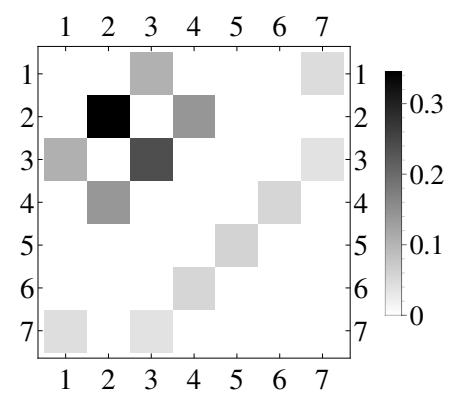

Figure 2.136. DFT, rupture 


\begin{tabular}{|l|l|}
\hline$G$ & 0.333 \\
$S$ & 100 \\
$A$ & 0 \\
$E$ & 0.0001 \\
$M$ & 35.1 \\
$\mathrm{Pr}$ & 7.02 \\
$R a$ & 0 \\
Domain size & 3 \\
Wave number & 2 \\
Amplitude of perturbation & 5 \\
Rupture time & 1347.8 \\
Initial condition & Cosine initial condition \\
\hline
\end{tabular}

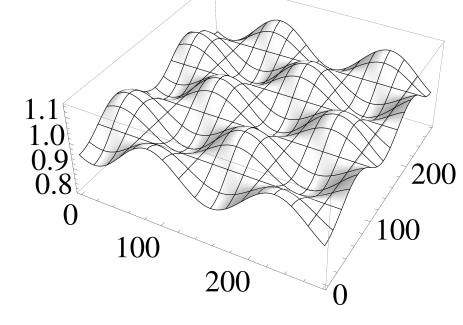

Figure 2.137. Initial condition

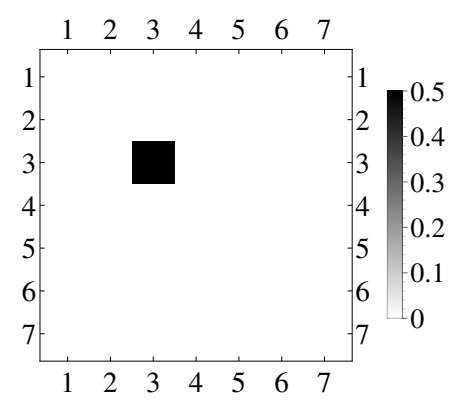

Figure 2.139. DFT, Initial condition

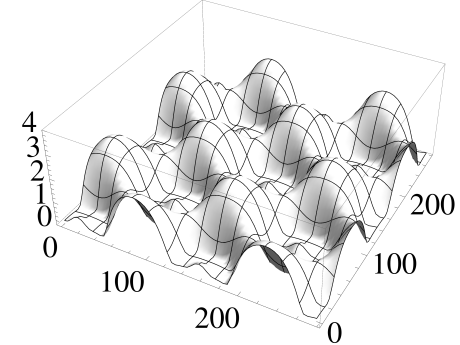

Figure 2.138. Rupture

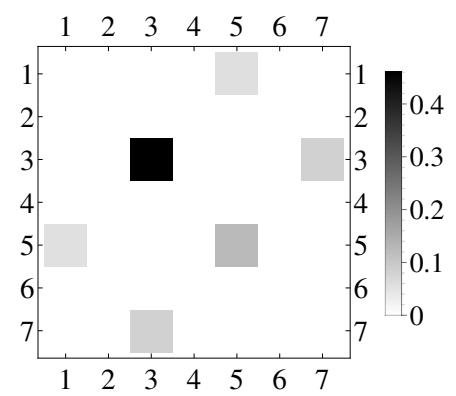

Figure 2.140. DFT, rupture 


\begin{tabular}{|l|l|}
\hline$G$ & 0.333 \\
$S$ & 100 \\
$A$ & 0 \\
$E$ & 0.0001 \\
$M$ & 35.1 \\
$\mathrm{Pr}$ & 7.02 \\
$R a$ & 0 \\
Domain size & 3 \\
Wave number & 3 \\
Amplitude of perturbation & 5 \\
Rupture time & 1850.4 \\
Initial condition & Cosine initial condition \\
\hline
\end{tabular}

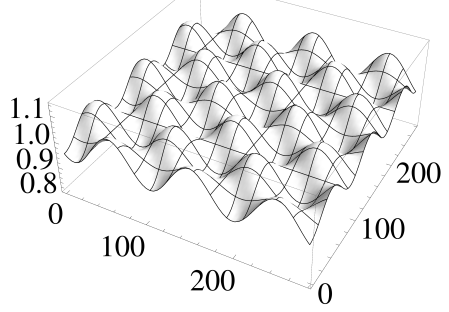

Figure 2.141. Initial condition

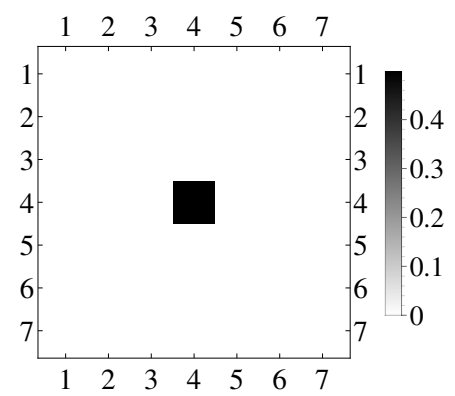

Figure 2.143. DFT, Initial condition

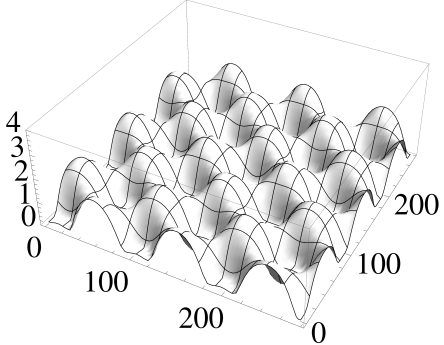

Figure 2.142. Rupture

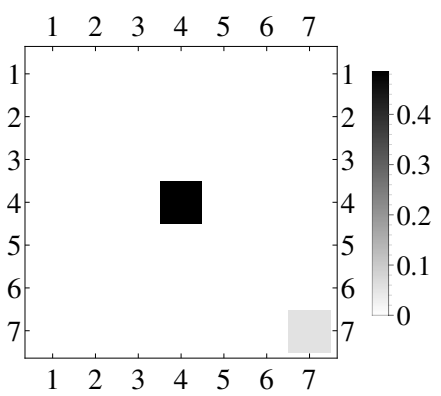

Figure 2.144. DFT, rupture 
Effect of initial conditions: Slowly Evaporating Mathematical fluid, $\mathbf{E}=0.0001, \mathbf{G}=0.0, L=\lambda_{\max }$ 


\begin{tabular}{|l|l|}
\hline$G$ & 0 \\
$S$ & 100 \\
$A$ & 0 \\
$E$ & 0.0001 \\
$M$ & 35.1 \\
$\mathrm{Pr}$ & 7.02 \\
$R a$ & 0 \\
Domain size & 3 \\
Wave number & 1 \\
Amplitude of perturbation & 5 \\
Rupture time & 1501.4 \\
Initial condition & Cosine initial condition \\
\hline
\end{tabular}

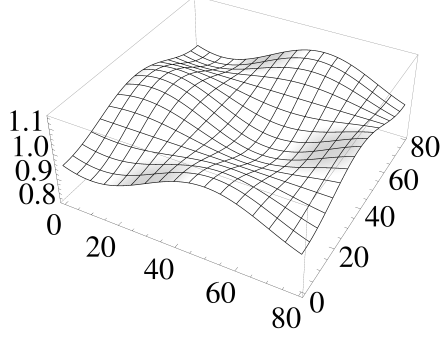

Figure 2.145. Initial condition

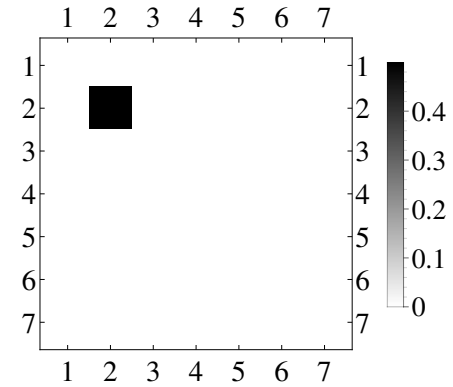

Figure 2.147. DFT, Initial condition

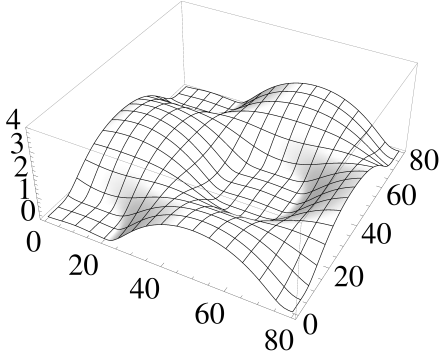

Figure 2.146. Rupture

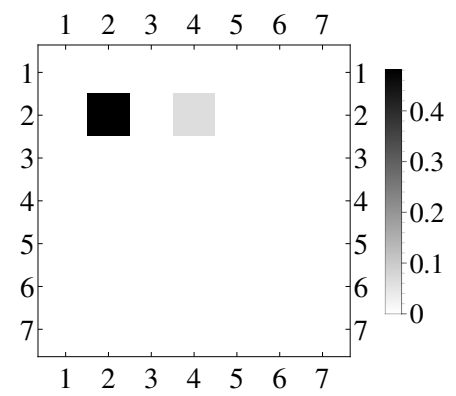

Figure 2.148. DFT, rupture 


\begin{tabular}{|l|l|}
\hline$G$ & 0 \\
$S$ & 100 \\
$A$ & 0 \\
$E$ & 0.0001 \\
$M$ & 35.1 \\
$\mathrm{Pr}$ & 7.02 \\
$R a$ & 0 \\
Domain size & 3 \\
Wave number & 1 \\
Amplitude of perturbation & 5 \\
Rupture time & 1084.7 \\
Initial condition & Cosine-rotated \\
\hline
\end{tabular}

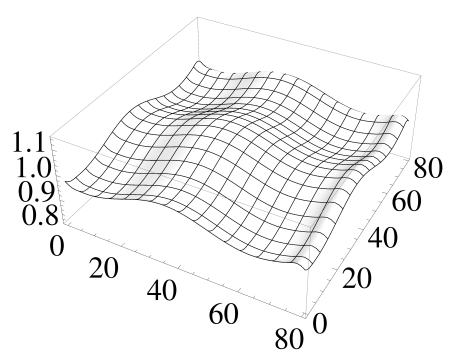

Figure 2.149. Initial condition

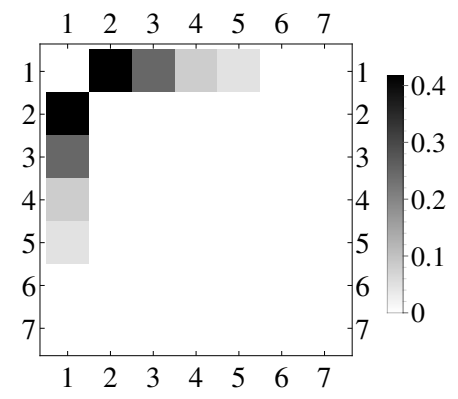

Figure 2.151. DFT, Initial condition

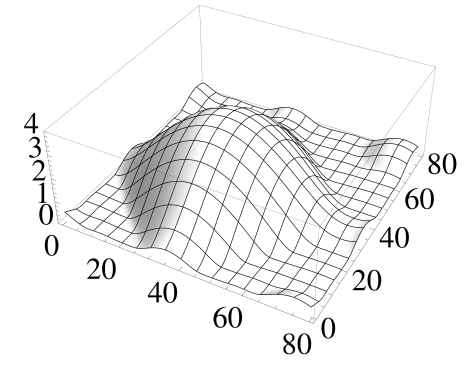

Figure 2.150. Rupture

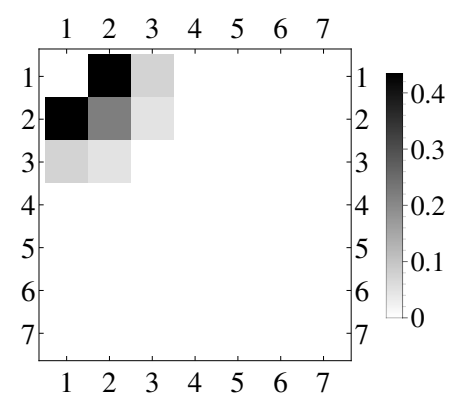

Figure 2.152. DFT, rupture 


\begin{tabular}{|l|l|}
\hline$G$ & 0 \\
$S$ & 100 \\
$A$ & 0 \\
$E$ & 0.0001 \\
$M$ & 35.1 \\
$\mathrm{Pr}$ & 7.02 \\
$R a$ & 0 \\
Domain size & 3 \\
Wave number & 1 \\
Amplitude of perturbation & 5 \\
Rupture time & 2124.4 \\
Initial condition & Cosine $\times$ Sine \\
\hline
\end{tabular}

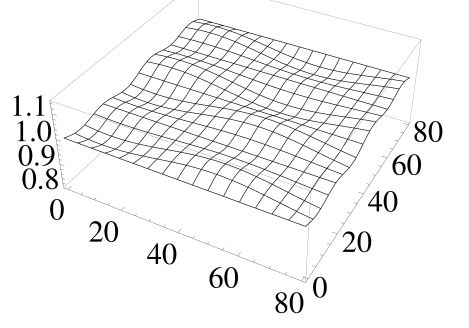

Figure 2.153. Initial condition

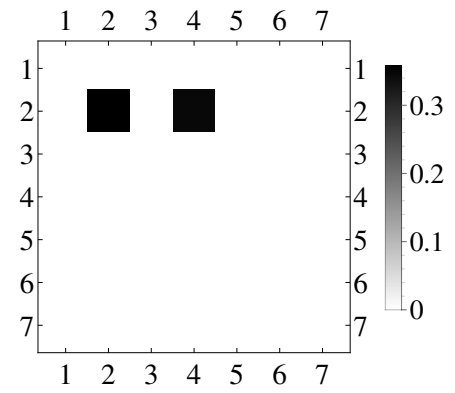

Figure 2.155. DFT, Initial condition

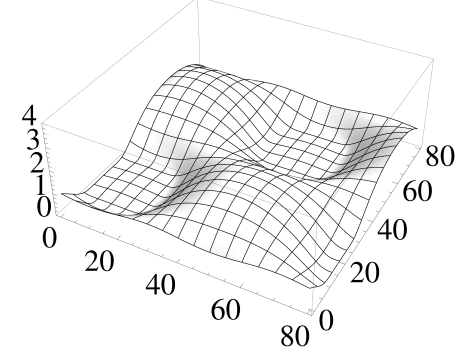

Figure 2.154. Rupture

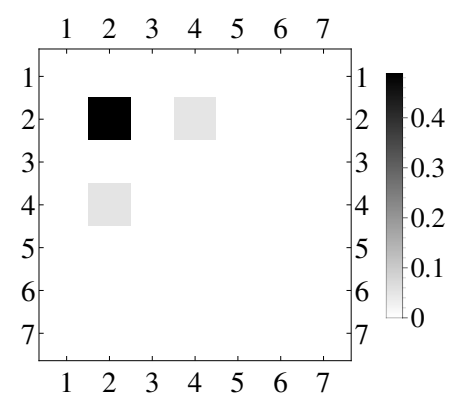

Figure 2.156. DFT, rupture 


\begin{tabular}{|l|l|}
\hline$G$ & 0 \\
$S$ & 100 \\
$A$ & 0 \\
$E$ & 0.0001 \\
$M$ & 35.1 \\
$\mathrm{Pr}$ & 7.02 \\
$R a$ & 0 \\
Domain size & 3 \\
Wave number & 1 \\
Amplitude of perturbation & 5 \\
Rupture time & 1013.3 \\
Initial condition & Cosine-squared \\
\hline
\end{tabular}

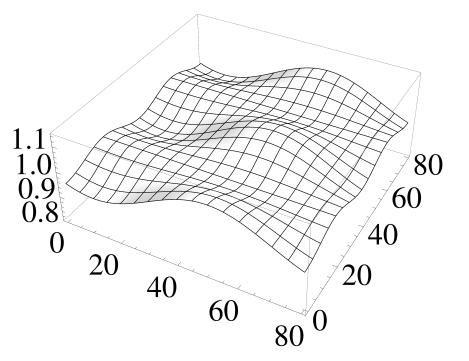

Figure 2.157. Initial condition

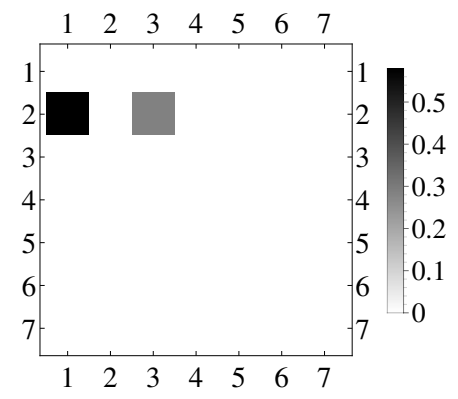

Figure 2.159. DFT, Initial condition

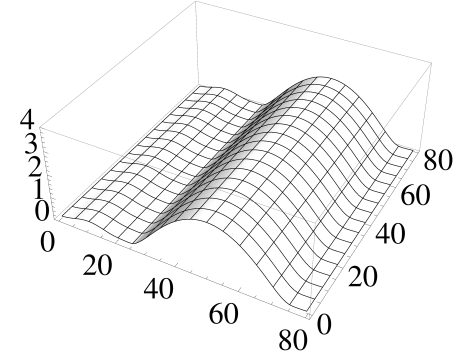

Figure 2.158. Rupture

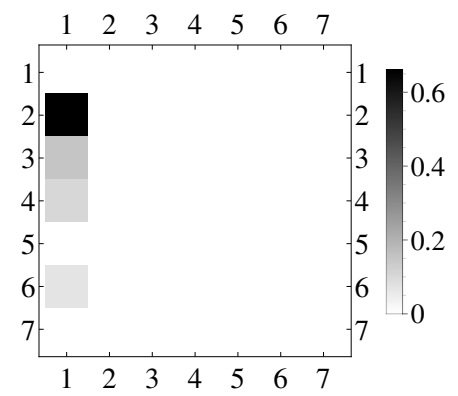

Figure 2.160. DFT, rupture 


\begin{tabular}{|l|l|}
\hline$G$ & 0 \\
$S$ & 100 \\
$A$ & 0 \\
$E$ & 0.0001 \\
$M$ & 35.1 \\
$\mathrm{Pr}$ & 7.02 \\
$R a$ & 0 \\
Domain size & 3 \\
Wave number & 1 \\
Amplitude of perturbation & 5 \\
Rupture time & 942.85 \\
Initial condition & Cosine-Y \\
\hline
\end{tabular}

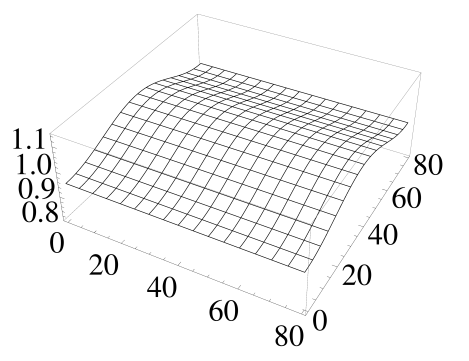

Figure 2.161. Initial condition

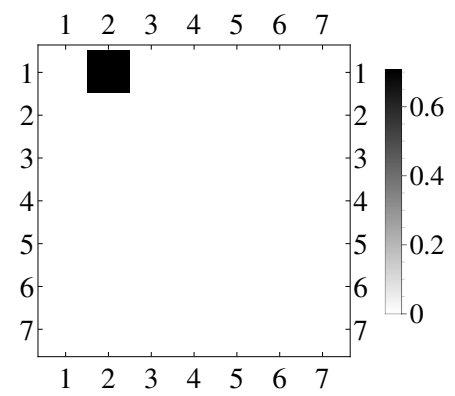

Figure 2.163. DFT, Initial condition

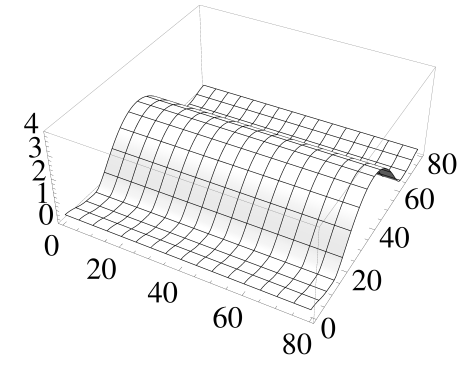

Figure 2.162. Rupture

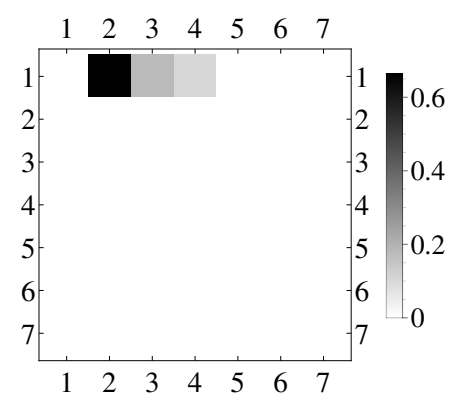

Figure 2.164. DFT, rupture 


\begin{tabular}{|l|l|}
\hline$G$ & 0 \\
$S$ & 100 \\
$A$ & 0 \\
$E$ & 0.0001 \\
$M$ & 35.1 \\
$\mathrm{Pr}$ & 7.02 \\
$R a$ & 0 \\
Domain size & 3 \\
Wave number & 1 \\
Amplitude of perturbation & 5 \\
Rupture time & 1501.8 \\
Initial condition & Sine initial condition \\
\hline
\end{tabular}

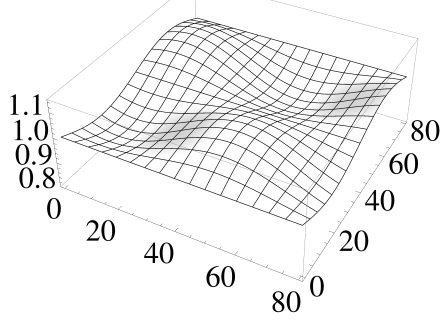

Figure 2.165. Initial condition

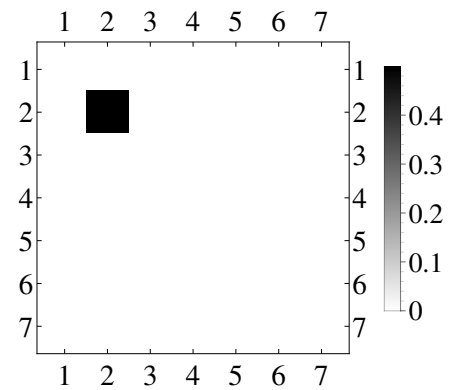

Figure 2.167. DFT, Initial condition

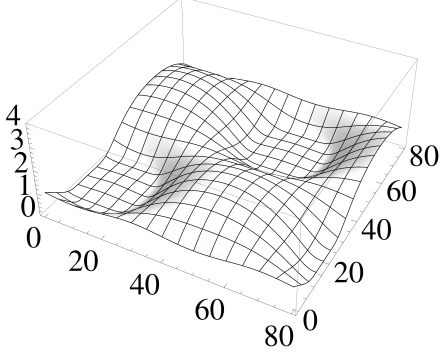

Figure 2.166. Rupture

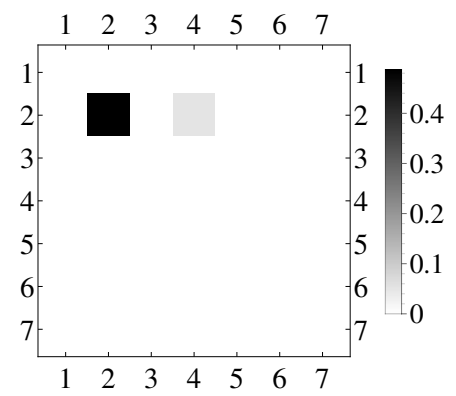

Figure 2.168. DFT, rupture 
Effect of initial conditions: Slowly Evaporating Mathematical fluid, $\mathrm{E}=\mathbf{0 . 0 0 0 1}, \mathrm{G}=\mathbf{0 . 0}, L=2 \lambda_{\max }$ 


\begin{tabular}{|l|l|}
\hline$G$ & 0 \\
$S$ & 100 \\
$A$ & 0 \\
$E$ & 0.0001 \\
$M$ & 35.1 \\
$\mathrm{Pr}$ & 7.02 \\
$R a$ & 0 \\
Domain size & 3 \\
Wave number & 1 \\
Amplitude of perturbation & 5 \\
Rupture time & 1206.4 \\
Initial condition & Cosine initial condition \\
\hline
\end{tabular}
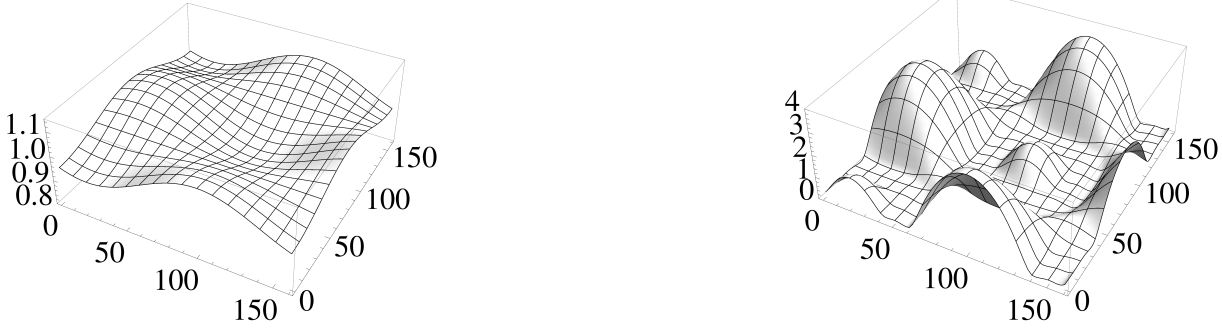

Figure 2.169. Initial condition

Figure 2.170. Rupture
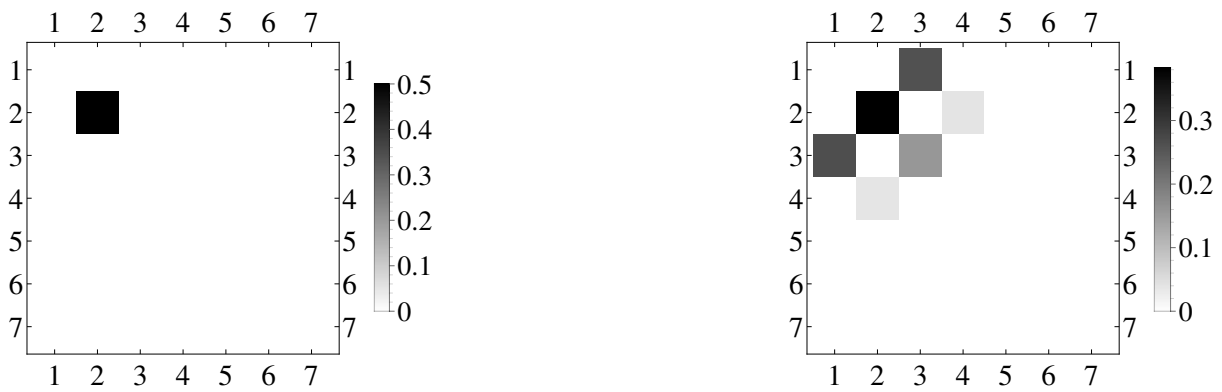

Figure 2.171. DFT, Initial condition

Figure 2.172. DFT, rupture 


\begin{tabular}{|l|l|}
\hline$G$ & 0 \\
$S$ & 100 \\
$A$ & 0 \\
$E$ & 0.0001 \\
$M$ & 35.1 \\
$\mathrm{Pr}$ & 7.02 \\
$R a$ & 0 \\
Domain size & 3 \\
Wave number & 1 \\
Amplitude of perturbation & 5 \\
Rupture time & 1102.5 \\
Initial condition & Cosine-rotated \\
\hline
\end{tabular}

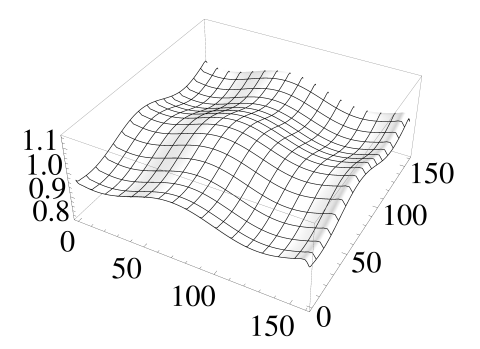

Figure 2.173. Initial condition

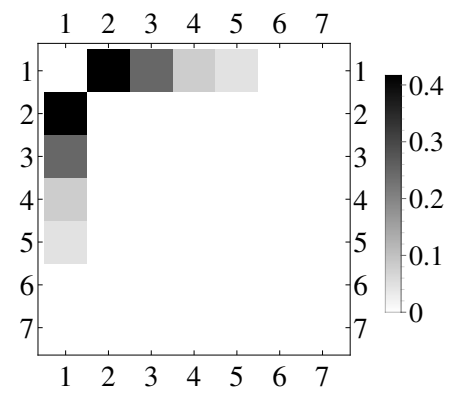

Figure 2.175. DFT, Initial condition

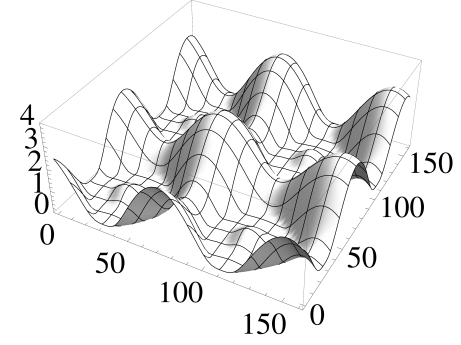

Figure 2.174. Rupture

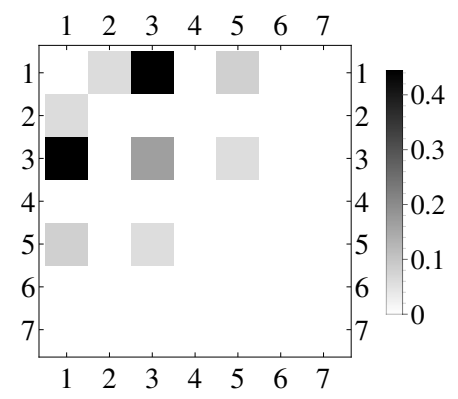

Figure 2.176. DFT, rupture 


\begin{tabular}{|l|l|}
\hline$G$ & 0 \\
$S$ & 100 \\
$A$ & 0 \\
$E$ & 0.0001 \\
$M$ & 35.1 \\
$\mathrm{Pr}$ & 7.02 \\
$R a$ & 0 \\
Domain size & 3 \\
Wave number & 1 \\
Amplitude of perturbation & 5 \\
Rupture time & 1628.8 \\
Initial condition & Cosine $\times$ Sine \\
\hline
\end{tabular}

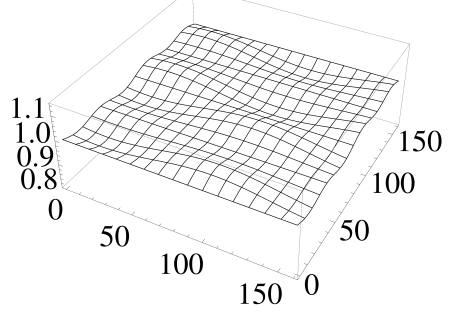

Figure 2.177. Initial condition

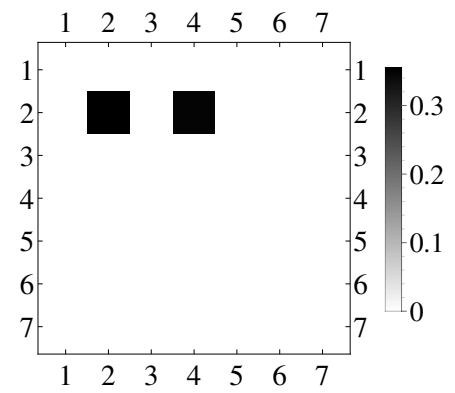

Figure 2.178. Rupture

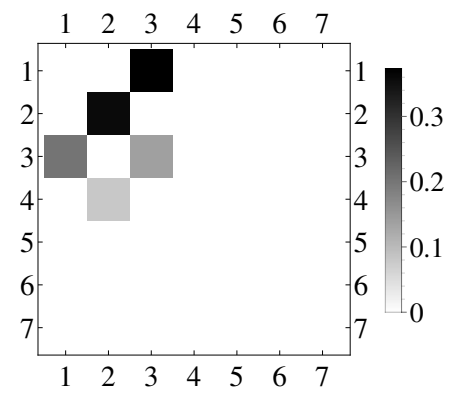

Figure 2.179. DFT, Initial condition

Figure 2.180. DFT, rupture 


\begin{tabular}{|l|l|}
\hline$G$ & 0 \\
$S$ & 100 \\
$A$ & 0 \\
$E$ & 0.0001 \\
$M$ & 35.1 \\
$\mathrm{Pr}$ & 7.02 \\
$R a$ & 0 \\
Domain size & 3 \\
Wave number & 1 \\
Amplitude of perturbation & 5 \\
Rupture time & 1021.7 \\
Initial condition & Cosine-squared \\
\hline
\end{tabular}

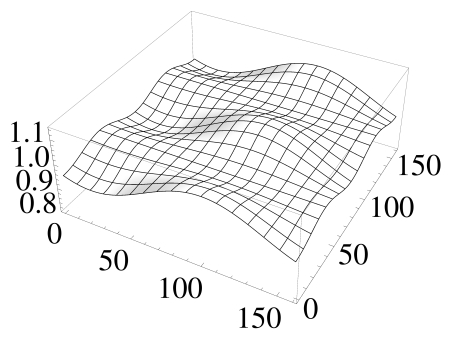

Figure 2.181. Initial condition

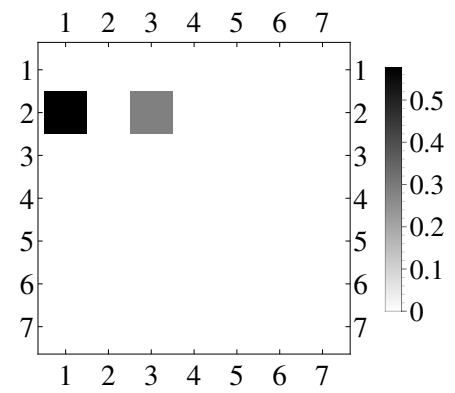

Figure 2.182. Rupture

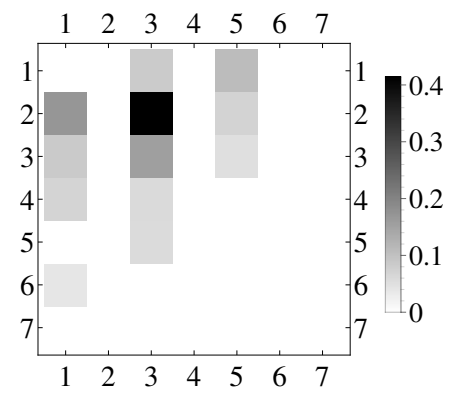

Figure 2.183. DFT, Initial condition

Figure 2.184. DFT, rupture 


\begin{tabular}{|l|l|}
\hline$G$ & 0 \\
$S$ & 100 \\
$A$ & 0 \\
$E$ & 0.0001 \\
$M$ & 35.1 \\
$\mathrm{Pr}$ & 7.02 \\
$R a$ & 0 \\
Domain size & 3 \\
Wave number & 1 \\
Amplitude of perturbation & 5 \\
Rupture time & 1208.4 \\
Initial condition & Sine initial condition \\
\hline
\end{tabular}

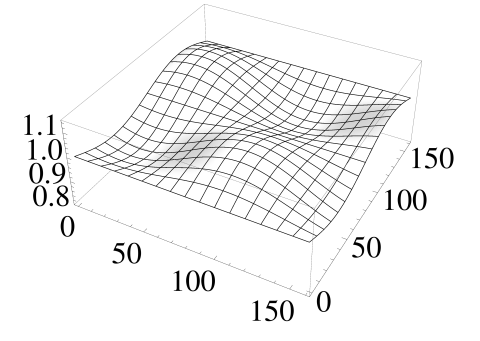

Figure 2.185. Initial condition

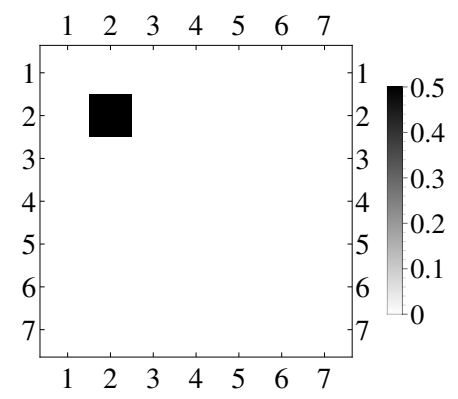

Figure 2.186. Rupture

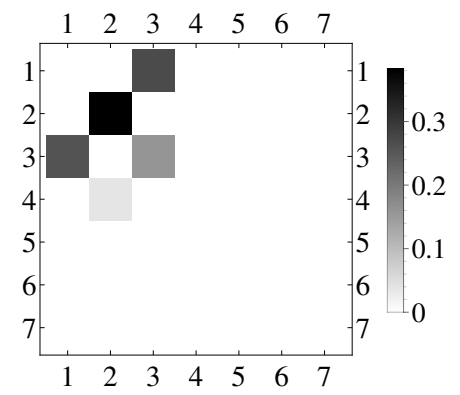

Figure 2.187. DFT, Initial condition

Figure 2.188. DFT, rupture 
Effect of initial conditions: Slowly Evaporating Mathematical fluid, $\mathrm{E}=\mathbf{0 . 0 0 0 1}, \mathrm{G}=\mathbf{0 . 0}, L=3 \lambda_{\max }$ 


\begin{tabular}{|l|l|}
\hline$G$ & 0 \\
$S$ & 100 \\
$A$ & 0 \\
$E$ & 0.0001 \\
$M$ & 35.1 \\
$\mathrm{Pr}$ & 7.02 \\
$R a$ & 0 \\
Domain size & 3 \\
Wave number & 1 \\
Amplitude of perturbation & 5 \\
Rupture time & 1674. \\
Initial condition & Cosine initial condition \\
\hline
\end{tabular}

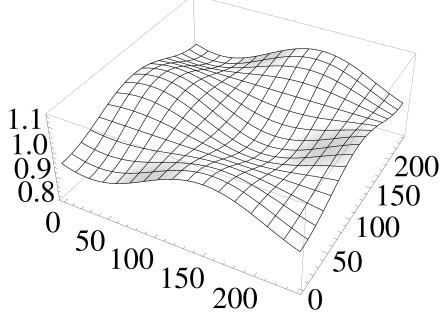

Figure 2.189. Initial condition

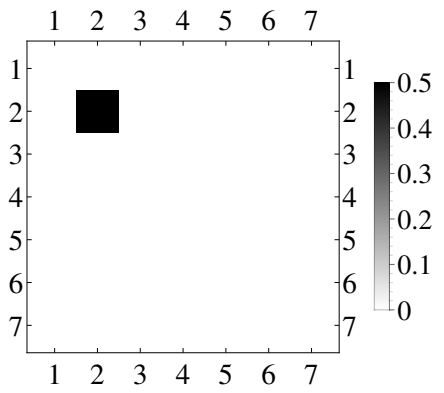

Figure 2.191. DFT, Initial condition

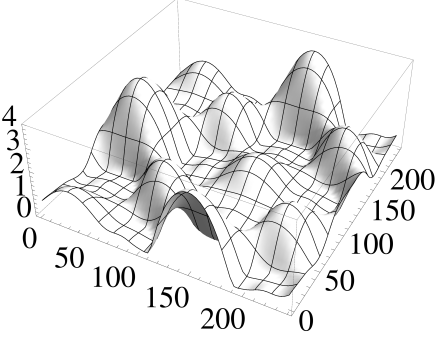

Figure 2.190. Rupture

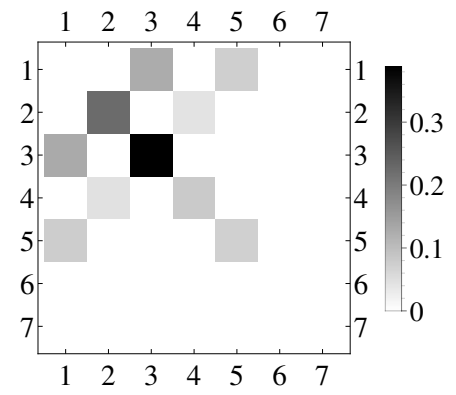

Figure 2.192. DFT, rupture 


\begin{tabular}{|l|l|}
\hline$G$ & 0 \\
$S$ & 100 \\
$A$ & 0 \\
$E$ & 0.0001 \\
$M$ & 35.1 \\
$\mathrm{Pr}$ & 7.02 \\
$R a$ & 0 \\
Domain size & 3 \\
Wave number & 1 \\
Amplitude of perturbation & 5 \\
Rupture time & 1191. \\
Initial condition & Cosine-rotated \\
\hline
\end{tabular}

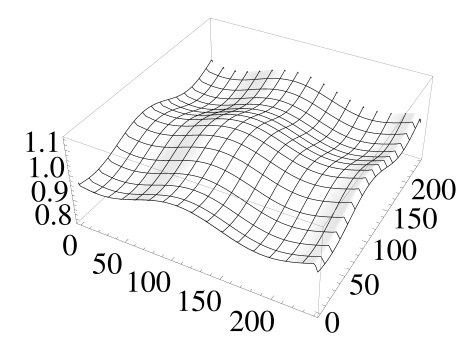

Figure 2.193. Initial condition

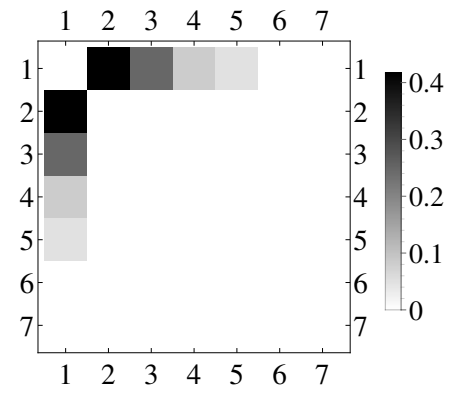

Figure 2.195. DFT, Initial condition

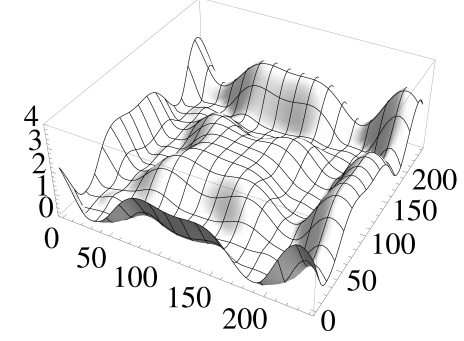

Figure 2.194. Rupture

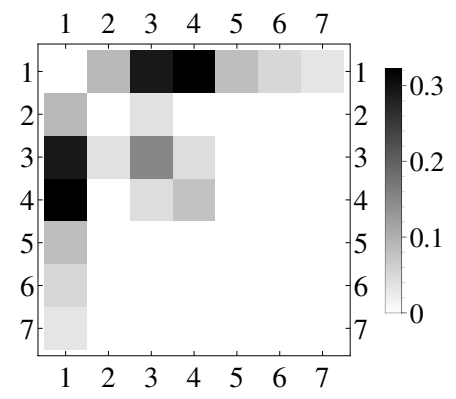

Figure 2.196. DFT, rupture 


\begin{tabular}{|l|l|}
\hline$G$ & 0 \\
$S$ & 100 \\
$A$ & 0 \\
$E$ & 0.0001 \\
$M$ & 35.1 \\
$\mathrm{Pr}$ & 7.02 \\
$R a$ & 0 \\
Domain size & 3 \\
Wave number & 1 \\
Amplitude of perturbation & 5 \\
Rupture time & 1163.1 \\
Initial condition & Cosine $\times$ Sine \\
\hline
\end{tabular}

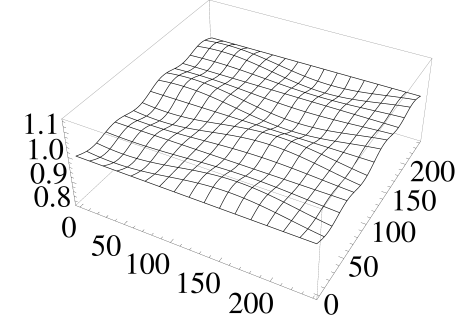

Figure 2.197. Initial condition

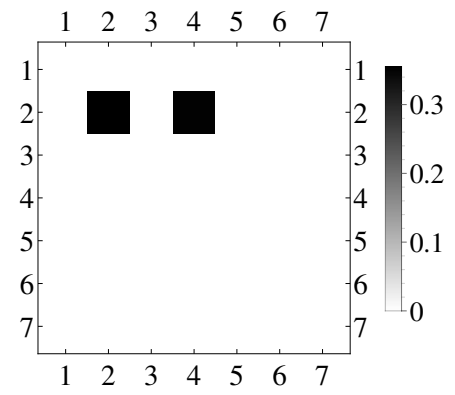

Figure 2.199. DFT, Initial condition

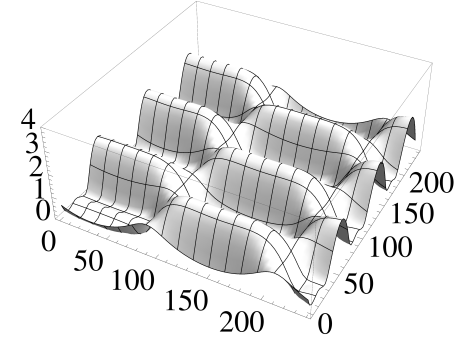

Figure 2.198. Rupture

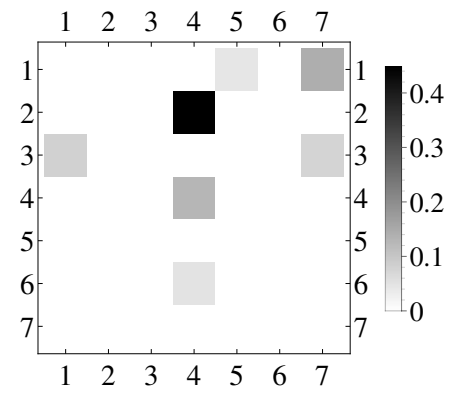

Figure 2.200. DFT, rupture 


\begin{tabular}{|l|l|}
\hline$G$ & 0 \\
$S$ & 100 \\
$A$ & 0 \\
$E$ & 0.0001 \\
$M$ & 35.1 \\
$\mathrm{Pr}$ & 7.02 \\
$R a$ & 0 \\
Domain size & 3 \\
Wave number & 1 \\
Amplitude of perturbation & 5 \\
Rupture time & 1163.1 \\
Initial condition & Cosine-squared \\
\hline
\end{tabular}

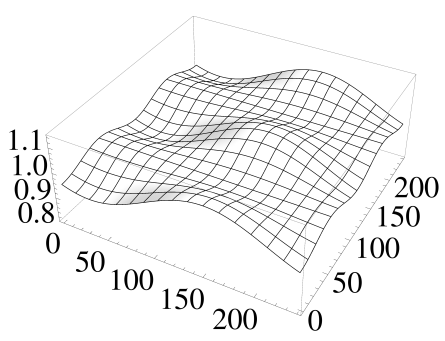

Figure 2.201. Initial condition

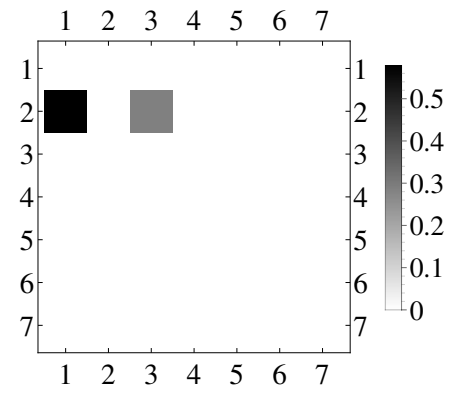

Figure 2.203. DFT, Initial condition

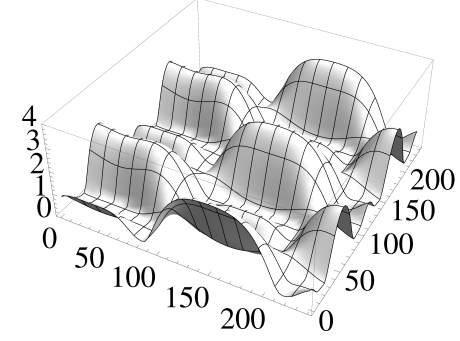

Figure 2.202. Rupture

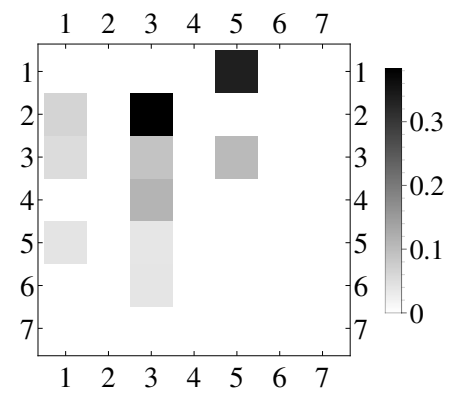

Figure 2.204. DFT, rupture 


\begin{tabular}{|l|l|}
\hline$G$ & 0 \\
$S$ & 100 \\
$A$ & 0 \\
$E$ & 0.0001 \\
$M$ & 35.1 \\
$\mathrm{Pr}$ & 7.02 \\
$R a$ & 0 \\
Domain size & 3 \\
Wave number & 1 \\
Amplitude of perturbation & 5 \\
Rupture time & 1319. \\
Initial condition & Cosine-Y \\
\hline
\end{tabular}

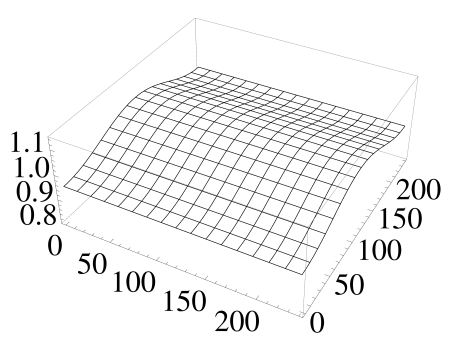

Figure 2.205. Initial condition

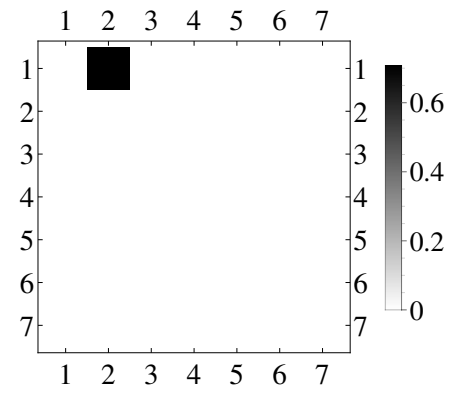

Figure 2.207. DFT, Initial condition

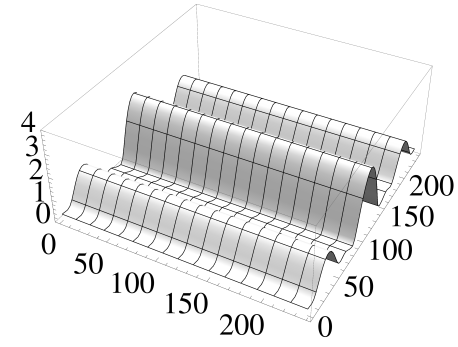

Figure 2.206. Rupture

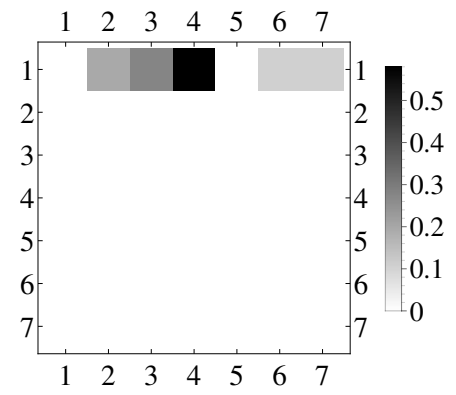

Figure 2.208. DFT, rupture 


\begin{tabular}{|l|l|}
\hline$G$ & 0 \\
$S$ & 100 \\
$A$ & 0 \\
$E$ & 0.0001 \\
$M$ & 35.1 \\
$\mathrm{Pr}$ & 7.02 \\
$R a$ & 0 \\
Domain size & 3 \\
Wave number & 1 \\
Amplitude of perturbation & 5 \\
Rupture time & 2346.6 \\
Initial condition & Sine initial condition \\
\hline
\end{tabular}

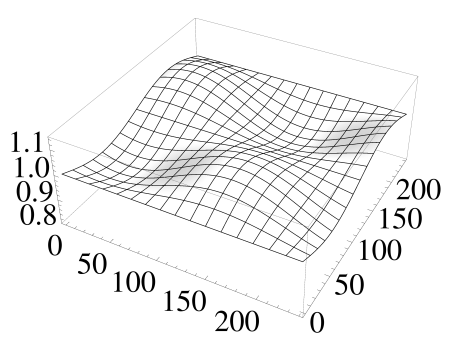

Figure 2.209. Initial condition

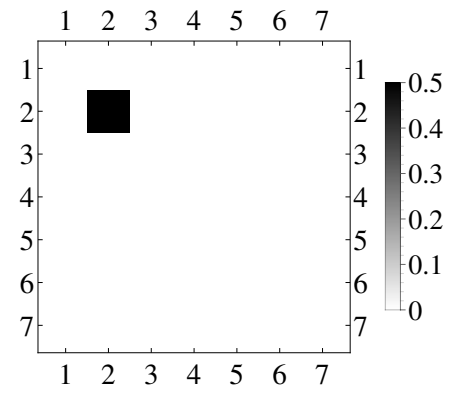

Figure 2.211. DFT, Initial condition

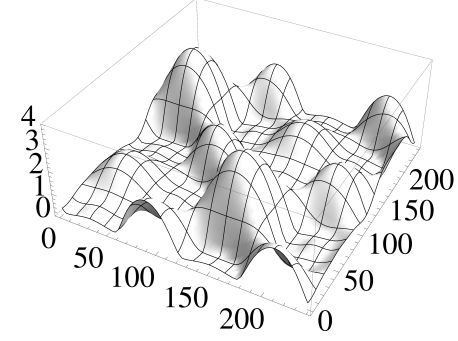

Figure 2.210. Rupture

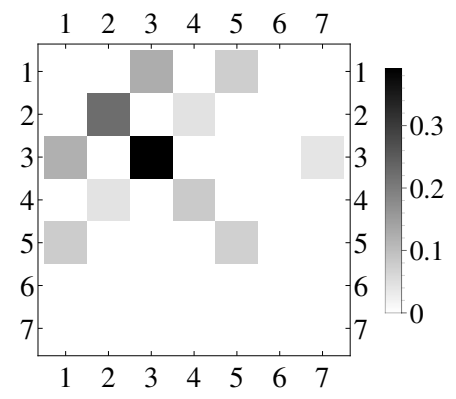

Figure 2.212. DFT, rupture 
Evaporating 2.35mm thick Dicholormethane film in zero gravity 


\begin{tabular}{|l|l|}
\hline$G$ & 0 \\
$S$ & 143393 \\
$A$ & 0 \\
$E$ & 0.0086 \\
$M$ & 122363 \\
$\mathrm{Pr}$ & 3.9 \\
$R a$ & 0 \\
Domain size & 1 \\
Wave number & 1 \\
Amplitude of perturbation & 5 \\
Rupture time & 36693. or 4 seconds \\
Initial condition & Cosine initial condition \\
\hline
\end{tabular}

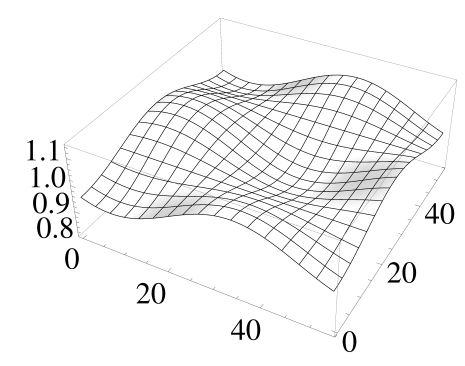

Figure 2.213. Initial condition

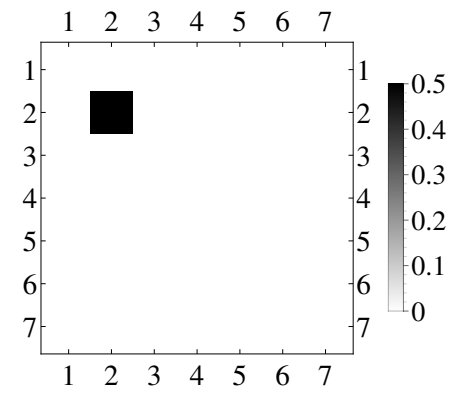

Figure 2.215. DFT, Initial condition

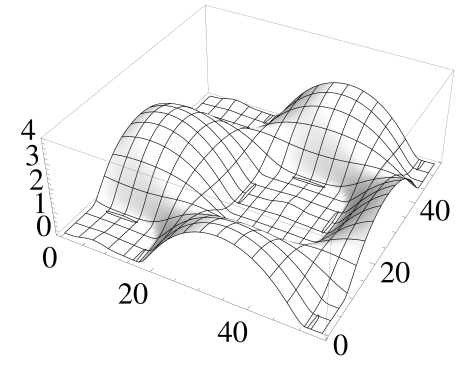

Figure 2.214. Rupture

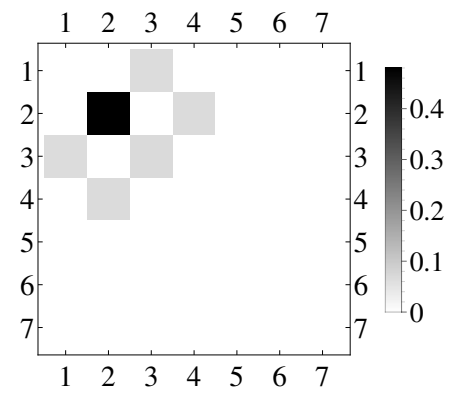

Figure 2.216. DFT, rupture 
Evaporating 2.35mm thick Dicholormethane film in Earth gravity 


\begin{tabular}{|l|l|}
\hline$G$ & $4.113 \mathrm{e} 6$ \\
$S$ & 143393 \\
$A$ & 0 \\
$E$ & 0.0086 \\
$M$ & 122363 \\
$\mathrm{Pr}$ & 3.9 \\
$R a$ & 0 \\
Domain size & 1 \\
Wave number & 1 \\
Amplitude of perturbation & 5 \\
Rupture time & $3.3122 \mathrm{e} 5$ or 57 seconds \\
Initial condition & Cosine initial condition \\
\hline
\end{tabular}

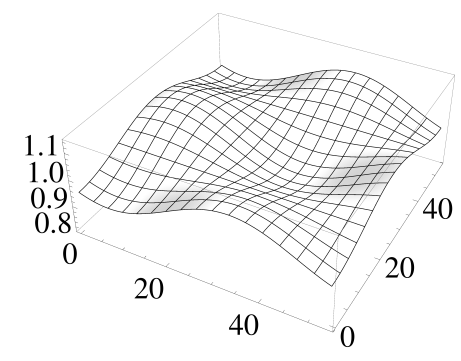

Figure 2.217. Initial condition

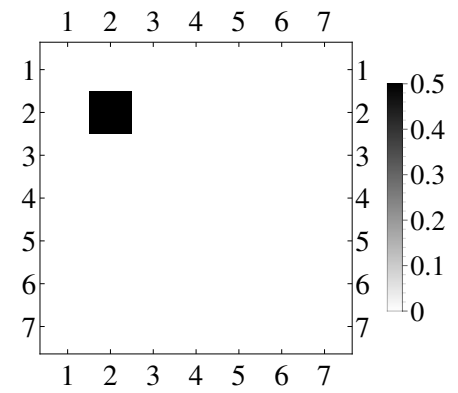

Figure 2.219. DFT, Initial condition

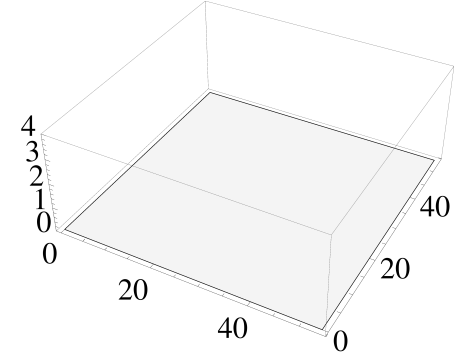

Figure 2.218. Rupture

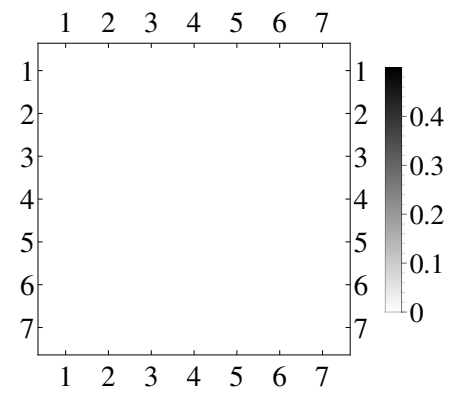

Figure 2.220. DFT, rupture 
Random perturbations, $2.35 \mathrm{~mm}$ DCM, $\mathbf{g}=0.0 \mathrm{~m} / \mathrm{s}^{2}$ in a domain size where $L=n \lambda_{\max }, n=$ non whole number 


\begin{tabular}{|l|l|}
\hline$G$ & 0.0 \\
$S$ & 143393 \\
$A$ & 0 \\
$E$ & 0.0086 \\
$M$ & 122363 \\
$\mathrm{Pr}$ & 3.9 \\
$R a$ & 0 \\
Domain size & 2.318 \\
Wave number & $\mathrm{n} / \mathrm{a}$ \\
Amplitude of perturbation & 5 \\
Rupture time & 391820 or 4.7 seconds \\
Initial condition & Uniform random perturbation \\
\hline
\end{tabular}

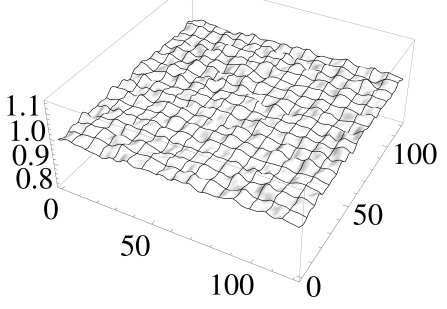

Figure 2.221. Initial condition

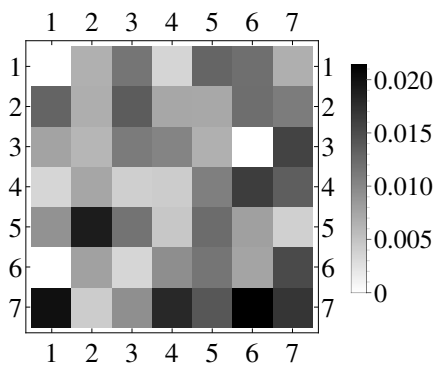

Figure 2.223. DFT, Initial condition

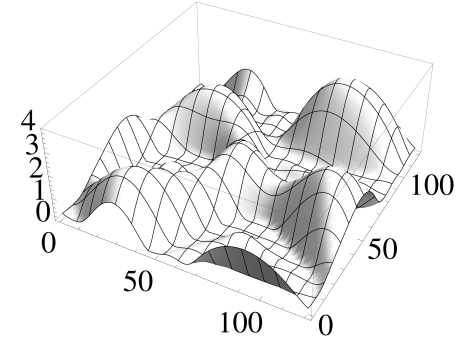

Figure 2.222. Rupture

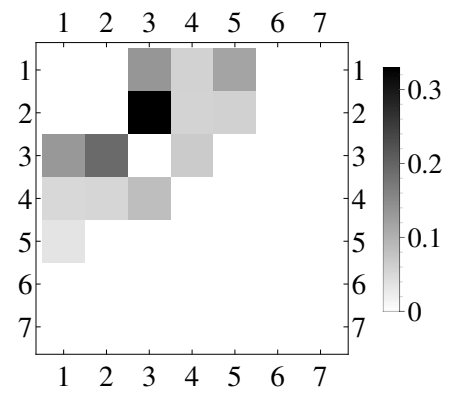

Figure 2.224. DFT, rupture 
Random perturbations, $2.35 \mathrm{~mm}$ DCM, $g=0.0 \mathrm{~m} / \mathrm{s}^{2}$ in a rectangular domain where $L x=2.238 \lambda_{\max }, L y=1.641 \lambda_{\max }$ 


\begin{tabular}{|l|l|}
\hline$G$ & 0.0 \\
$S$ & 143393 \\
$A$ & 0 \\
$E$ & 0.0086 \\
$M$ & 122363 \\
$\mathrm{Pr}$ & 3.9 \\
$R a$ & 0 \\
Domain size & 2.318 \\
Wave number & $\mathrm{n} / \mathrm{a}$ \\
Amplitude of perturbation & 5 \\
Rupture time & 378970 or 4.54 seconds \\
Initial condition & Uniform random perturbation \\
\hline
\end{tabular}

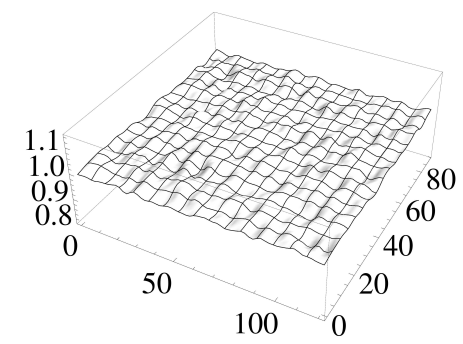

Figure 2.225. Initial condition

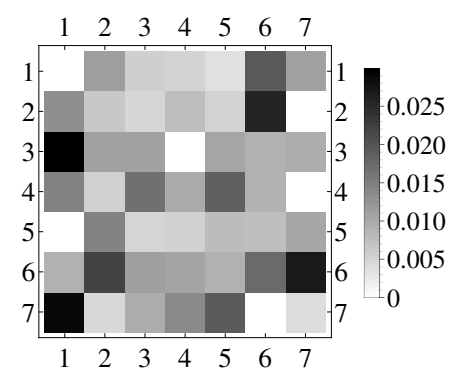

Figure 2.227. DFT, Initial condition

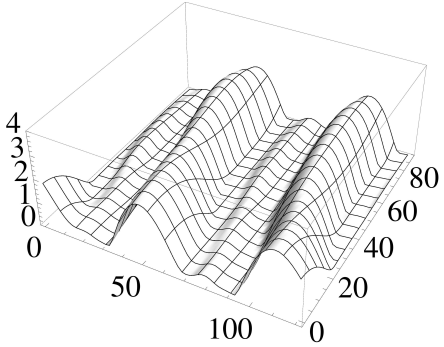

Figure 2.226. Rupture

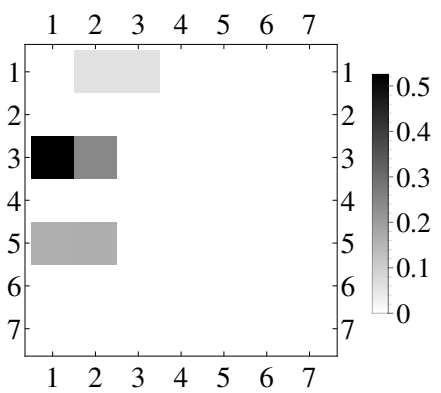

Figure 2.228. DFT, rupture 


\section{REFERENCES}

[1] S. Krishnamoorthy, B. Ramaswamy, and S. W. Joo. Spontaneous rupture of thin liquid films due to thermocapillarity: A full-scale direct numerical simulation. Physics of Fluids, 7(9):2291-2293, 1995.

[2] Alexander Oron. Nonlinear dynamics of three-dimensional long-wave Marangoni instability in thin liquid films. Physics of Fluids, 12(7):1633-1645, 2000.

[3] R. V. Craster and O. K. Matar. Dynamics and stability of thin liquid films. Reviews of Modern Physics, 81(3):1131-1198, August 2009.

[4] Nicholas P. Cheremisinoff. Industrial Solvents Handbook. Marcel Decker, second edition, 2003.

[5] Hüseyin Pehlivan and Mustafa Özdemir. Experimental and theoretical investigations of falling film evaporation. Heat and Mass Transfer, pages 1-9, January 2012.

[6] M. Moresi. Apple juice concentration by reverse osmosis and falling film evaporation. Preconcentration and drying of food materials, pages 61-76, 1988.

[7] Bruno Agostini, Matteo Fabbri, Jung E. Park, Leszek Wojtan, John R. Thome, and Bruno Michel. State of the Art of High Heat Flux Cooling Technologies. Heat Transfer Engineering, 28(4):258-281, 2007.

[8] J. Darabi. Development of a chip-integrated micro cooling device. Microelectronics Journal, 34(11):1067-1074, November 2003.

[9] Jackson B. Marcinichen, John R. Thome, and Bruno Michel. Cooling of microprocessors with micro-evaporation: A novel two-phase cooling cycle. International Journal of Refrigeration, 33(7):1264-1276, November 2010.

[10] Laurier L. Schramm and Jerry J. Novosad. The destabilization of foams for improved oil recovery by crude oils: Effect of the nature of the oil. Journal of Petroleum Science and Engineering, 7(1-2):77-90, April 1992. 
[11] V. Bergeron, M. E. Fagan, and C. J. Radke. Generalized entering coefficients: a criterion for foam stability against oil in porous media. Langmuir, 9(7):17041713, July 1993.

[12] H. Benard. Les tourbillons cellulaires dans une nappe liquide. Rev. Gen. Sci. Pure Appl., 11:679-686, 1900.

[13] James Thomson. On certain curious motions observable at the surfaces of wine and other alcoholic liquors. Phil. Mag. Ser., 10(67):330-333, 1855.

[14] Rayleigh. On convective currents in a horizontal layer of fluid when the higher temperature is on the under side. Philo. Mag. Series, 32(6):529-546, 1916.

[15] H. Jeffreys. The stability of a layer of fluid heated below. Philo. Mag. Series, 2 (10):833-844, 1926.

[16] Anne Pellew and R. V. Southwell. On Maintained Convective Motion in a Fluid Heated from below. Proceedings of the Royal Society of London. Series A, Mathematical and Physical Sciences, 176(966):312-343, 1940.

[17] J. R. A. Pearson. On convection cells induced by surface tension. Journal of Fluid Mechanics Digital Archive, 4(05):489-500, 1958.

[18] Harvey J. Palmer. The hydrodynamic stability of rapidly evaporating liquids at reduced pressure. Journal of Fluid Mechanics Digital Archive, 75(03):487-511, 1976 .

[19] M. R. E. Proctor. Planform selection by finite-amplitude thermal convection between poorly conducting slabs. Journal of Fluid Mechanics Digital Archive, 113(-1):469-485, 1981.

[20] D. A. Nield. Surface tension and buoyancy effects in cellular convection. Journal of Fluid Mechanics, 19(03):341-352, 1964.

[21] L. E. Scriven and C. V. Sternling. On cellular convection driven by surfacetension gradients - Effects of mean surface tension and surface viscosity. Journal of Fluid Mechanics, 19(Part 3), 1964.

[22] M. B. Williams and S. H. Davis. Nonlinear theory of film rupture. Journal of Colloid and Interface Science, 90(2):220-228, 1982.

[23] D. J. Benny. Long waves on liquid films. Journal of Mathematics and Physics, 45(2):150-155, 1966. 
[24] D. J. Benny. Long non-linear waves in fluid flows (Long finite amplitude wave motions and interactions in inviscid fluid flows). Journal of Mathematics and Physics, 45:52-63, 1966.

[25] J. P. Burelbach, S. G. Bankoff, and S. H. Davis. Nonlinear stability of evaporating/condensing films. Journal of Fluid Mechanics, 195:463-494, 1988.

[26] A. Sharma and E. Ruckenstein. An analytical nonlinear theory of thin film rupture and its application to wetting films. Journal of Colloid and Interface Science, 113(2), 1986.

[27] J. P. Burelbach, S. G. Bankoff, and S. H. Davis. Steady thermocapillary flows of thin liquid layers. II. Experiment. Physics of Fluids A: Fluid Dynamics, 2(3): 322-333, 1990.

[28] M. J. Tan, S. G. Bankoff, and S. H. Davis. Steady thermocapillary flows of thin liquid layers. I. Theory. Physics of Fluids A: Fluid Dynamics, 2(3):313-321, 1990.

[29] G. K. Pradhan and P. C. Samal. Thermal stability of a fluid layer under variable body forces. Journal of Mathematical Analysis and Applications, 122(2), 1987.

[30] Brian Straughan. Convection in a variable gravity field. Journal of Mathematical Analysis and Applications, 140(2), 1989.

[31] Stephen J. Vanhook, Michael F. Schatz, J. B. Swift, W. D. McCormick, and Harry L. Swinney. Long-wavelength surface-tension-driven Benard convection: experiment and theory. Journal of Fluid Mechanics, 345(1):45-78, 1997.

[32] I. Ostrach. Low gravity fluid flows. Annual Review of Fluid Mechanics, 14: 313-345, January 1982.

[33] Alexander Oron and Yoav Peles. Stabilization of thin liquid films by internal heat generation. Physics of Fluids, 10(3):537-539, 1998.

[34] Roman O. Grigoriev. Control of evaporatively driven instabilities of thin liquid films. Physics of Fluids, 14(6):1895-1909, 2002.

[35] Alexander Oron. Nonlinear dynamics of irradiated thin volatile liquid films. Physics of Fluids, 12(1):29-41, 2000.

[36] Stergios G. Yiantsios and Brian G. Higgins. Rupture of thin films: Nonlinear stability analysis. Journal of Colloid and Interface Sciences, 147(2), 1991. 
[37] Shang-Hwei Chang and Chi-Chuan Hwang. Rupture theory of thin power-law liquid film. Journal of Applied Physics, 74(4), 1993.

[38] Ming-Cheng Wu and Chi-Chuan Hwang. Nonlinear theory of film rupture with viscosity variation. International Communications in Heat and Mass Transfer, 18(5), 1991.

[39] Eric Sultan, Areski Boudaoud, and Martine B. Amar. Evaporation of a thin film: diffusion of the vapour and Marangoni instabilities. Journal of Fluid Mechanics, 543:183-202, 2005.

[40] Igor L. Kliakhandler, Stephen H. Davis, and S. George Bankoff. The effect of heat conduction in the vapor on the dynamics of downflowing condensate. Physics of Fluids, 14(1):150-157, 2002.

[41] Pijush Kundu. Fluid Mechanics. Academic Press Inc., 1990.

[42] S. Chandrashekhar. Hydrodynamic and Hydromagnetic Stability. Dover, 1961.

[43] Alexander Oron, Stephen H. Davis, and S. George Bankoff. Long-scale evolution of thin liquid films. Rev. Mod. Phys., 69(3):931-980, Jul 1997.

[44] S.H. Davis. Rupture of thin liquid films. Waves on surfaces, 1987.

[45] A.C. Hindmarsh. LSODE: Livermore solver for ordinary differential equations. Technical report, Lawrence Livermore National Laboratory, March 1987.

[46] A. Struthers, R.W. Kolkka, J.S. Allen, and A.D. Narendranath. Accurately Resolving Rupture in 4th order Partial Differential Equations Modeling Thin Films Using NDSolve. Wolfram Technology Conference 2010, 2010.

[47] Stephen J. VanHook, Michael F. Schatz, William D. McCormick, J. B. Swift, and Harry L. Swinney. Long-Wavelength Instability in Surface-Tension-Driven Bénard Convection. Phys. Rev. Lett., 75(24):4397-4400, Dec 1995.

[48] Leslie Y. Yeo, Richard V. Craster, and Omar K. Matar. Marangoni instability of a thin liquid film resting on a locally heated horizontal wall. Phys. Rev. E, 67:056315, May 2003.

[49] S. W. Joo, S. H. Davis, and S. G. Bankoff. Long-wave instabilities of heated falling films: two-dimensional theory of uniform layers. Journal of Fluid Mechanics, 230(-1):117-146, 1991.

[50] W. Boos and A. Thess. Cascade of structures in long-wavelength Marangoni instability. Physics of Fluids, 11:1484-1494, June 1999. 
[51] Ricardo Becerril, Stephen J. Hook, and J. B. Swift. The influence of interface profile on the onset of long-wavelength Marangoni convection. Physics of Fluids, 10(12):3230-3232, 1998.

[52] A. J. Petsi and V. N. Burganos. Stokes flow inside an evaporating liquid line for any contact angle. Physical Review E, 78:036324+, September 2008.

[53] Jeramy Kimball. Interfacial instability, convective structure and heat transfer in liquids undergoing phase change. PhD thesis, University of Washington, Seattle, 2010.

[54] Peter C. Wayner. The effect of interfacial mass transport on flow in thin liquid films. Colloids and Surfaces, 52:71-84, 1991.

[55] Robert W. Schrage. A Theoretical Study of Interphase Mass Transfer. Columbia University Press, New York, 1953. 